University of Louisville

ThinkIR: The University of Louisville's Institutional Repository

Electronic Theses and Dissertations

$12-2012$

\title{
Ecological interactions affecting the role of mosquito larvae on dengue virus transmission.
}

Jeffrey J. Bara 1984-

University of Louisville

Follow this and additional works at: https://ir.library.louisville.edu/etd

\section{Recommended Citation}

Bara, Jeffrey J. 1984-, "Ecological interactions affecting the role of mosquito larvae on dengue virus transmission." (2012). Electronic Theses and Dissertations. Paper 70.

https://doi.org/10.18297/etd/70

This Doctoral Dissertation is brought to you for free and open access by ThinkIR: The University of Louisville's Institutional Repository. It has been accepted for inclusion in Electronic Theses and Dissertations by an authorized administrator of ThinkIR: The University of Louisville's Institutional Repository. This title appears here courtesy of the author, who has retained all other copyrights. For more information, please contact thinkir@louisville.edu. 


\title{
ECOLOGICAL INTERACTIONS AFFECTING THE ROLE OF MOSQUITO LARVAE ON DENGUE VIRUS TRANSMISSION
}

\author{
By \\ Jeffrey J. Bara \\ B.S., Western Illinois University, 2007

\begin{abstract}
A Dissertation
Submitted to the Faculty of the

College of Arts and Sciences of the University of Louisville in Partial Fulfillment of the Requirements

for the Degree of
\end{abstract}

Doctor of Philosophy

Department of Biology

University of Louisville

Louisville, Kentucky

December 2012 


\title{
ECOLOGICAL INTERACTIONS AFFECTING THE ROLE OF MOSQUITO LARVAE ON DENGUE VIRUS TRANSMISSION
}

\author{
By \\ Jeffrey J. Bara \\ B.S., Western Illinois University, 2007
}

A Dissertation Approved on

November 16, 2012

by the following Dissertation Committee:

Dr. Susanna Remold

Dissertation Director

Dr. Thomas M. Clark

Dr. Karen Golemboski

Dr. Michael H. Perlin

Dr. Sarah Emery 


\section{DEDICATION}

This dissertation is dedicated to my parents

Ronald and Karen Bara

for their unwavering support and encouragement. 


\section{ACKNOWLEDGEMENTS}

I would like to thank my advisor Dr. Susanna Remold for all of her teaching and guidance over the past five years. Dr. Remold, I am truly appreciative all of the support you have given me over the last five years and I look forward to continued scientific collaborations. I would also like to thank my committee members Dr. Thomas Clark, Dr. Michael Perlin, Dr. Sarah Emery, and Dr. Karen Golemboski, for all of their insightful comments and assistance over the past five years.

To all Remold lab members past and present I am thankful for the many ways in which you have assisted me throughout my graduate career and for your companionship. You made coming into the lab each day enjoyable and interesting, LLAP Remoldians.

Special thanks to Tim Herndon and the F.B Purnell Sausage Company for providing me free hog's blood to maintain my mosquito colonies and to Mark Eid and Megan Purdy for driving me out to Simpsonville early in the morning to pick it up. I would like to thank Dr. Kathy Hanley and her lab at New Mexico State for all of their technical assistance and for providing several viral strains. I would also like to thank Dr. Robert Tesh and the World Arbovirus Collection at the University of Texas Medical Branch for providing monoclonal antibodies and viral strains.

Last but not least, I would like to thank my wife, Allie Smith for her support and for her participation in mosquito collecting. I would also like to thank my mother and father in law, Jeff Smith and Anne Steinbock for all of their support over the past 5 years, and the whole Steinbock family for making me feel at home in Louisville. 


\section{ABSTRACT \\ ECOLOGICAL INTERACTIONS AFFECTING THE ROLE OF MOSQUITO LARVAE ON DENGUE VIRUS TRANSMISSION}

Jeffrey J. Bara

November 16, 2012

In the course of their life cycle, mosquitoes undergo an ontogenetic niche shift; immature larval development occurs within an aquatic habitat from which adult mosquitoes subsequently disperse into the terrestrial environment. While adult female mosquitoes transmit dengue to humans, the larval stage influences dengue virus transmission significantly in several ways. The biotic and abiotic conditions in which larvae develop influence epidemiologically important aspects of adult life history and influence the geographic distribution of mosquito species. Through vertical transmission, mosquitoes may serve as reservoir hosts for dengue during periods that are unfavorable for transmission to humans.

For my dissertation, I conducted research that investigated how environmental conditions experienced by larvae influence dengue transmission both directly and indirectly.

In chapter 1, I experimentally infected Aedes aegypti and Aedes albopictus larvae to determine their susceptibility to dengue and evaluate the potential for horizontal 
transmission within the larval habitat. I found that larvae are susceptible to dengue and that horizontal transmission could be possible.

In chapter 2 , I tested the hypothesis that dengue infection affected mosquito fitness. The effect of infection was sex dependent; infected males took significantly longer to develop and were significantly smaller, while infection in females was costfree. This result indicates that female larvae infected through vertical transmission are likely to be able to transmit dengue to humans as adults.

In chapter 3 and 4, I investigated how resources within the larval habitat influence development and competition. Using larval detritus as a resource, I studied its utilization within the habitat, and its influence on larval development and competition. I found that larval detritus was consumed rapidly and differently from another type of invertebrate detritus. When larval detritus was the main resource within the larval habitat, $A e$.

albopictus significantly outcompeted Ae. aegypti; Ae. aegypti were significantly smaller and took longer to develop when reared in containers with Ae. albopictus. This result indicates that interspecific competition with Ae. albopictus significantly decreases the population growth of $A e$. aegypti and may influence its ability to transmit dengue.

Overall, my results further demonstrate the influential role of mosquito larvae on dengue transmission and epidemiology. 


\section{TABLE OF CONTENTS}

PAGE

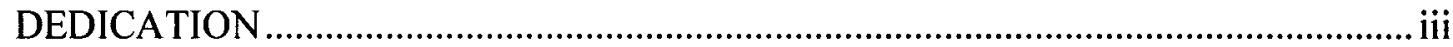

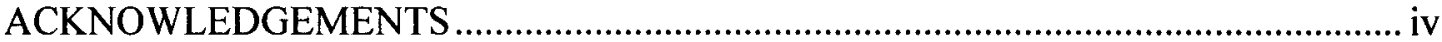

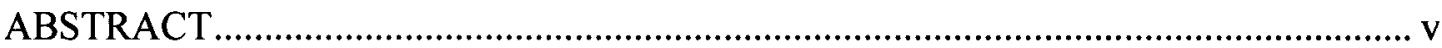

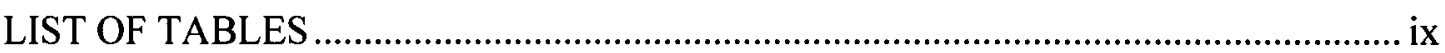

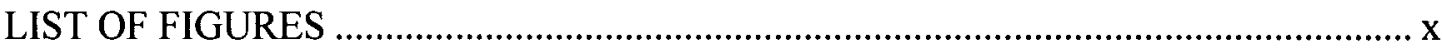

CHAPTER

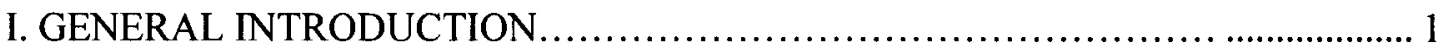

Dengue Virus and Mosquito Vectors...................................................................... 1

Dengue Emergence and Epidemiology ..................................................................... 3

Mosquitoes as a Potential Dengue Reservoir Host............................... 7

Dengue Transmission and Pathogenesis................................................ 10

Role of the Larval Development on Dengue Transmission..............................13

Effect of the Larval Environment on Adult Life History Traits.................................15

Effect of the Larval Environment of Mosquito Distribution......................................18

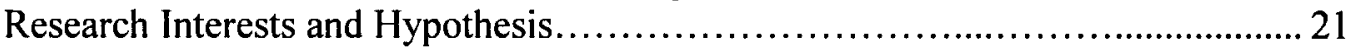

II. SUSCEPTIBILITY OF LARVAL $A E$. AEGYPTI AND AE. ALBOPICTUS TO

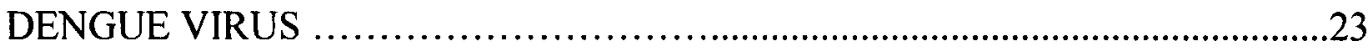

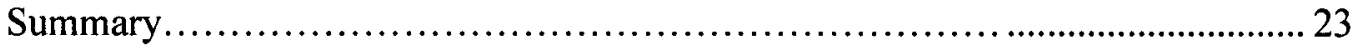

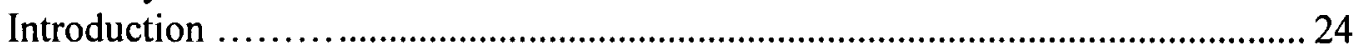

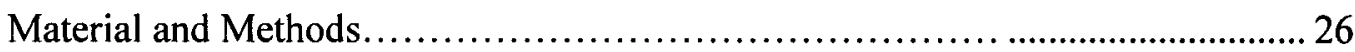

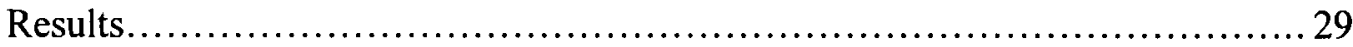

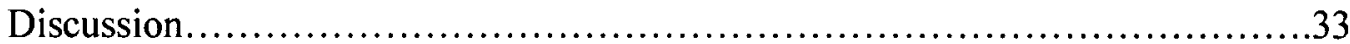

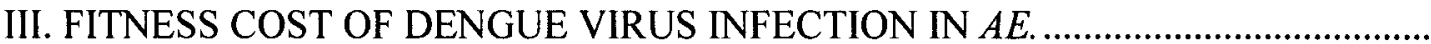

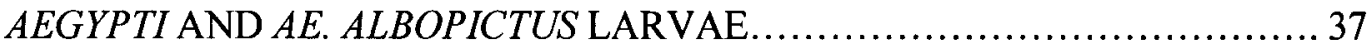

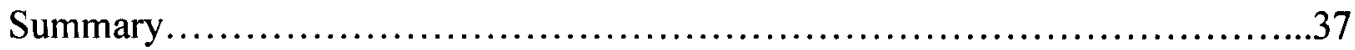

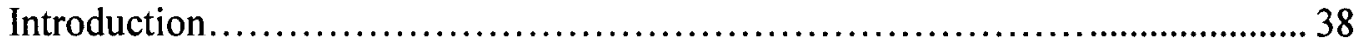

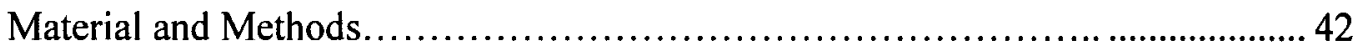

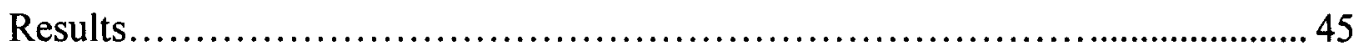

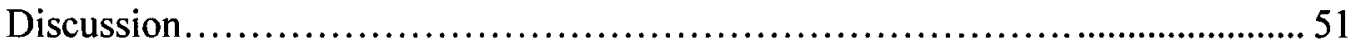


IV. UTILIZATION OF LARVAL DETRITUS WITHIN THE LARVAL HABITAT ... 55

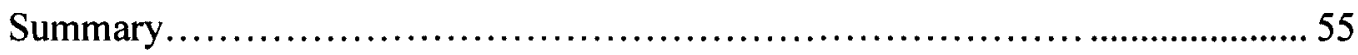

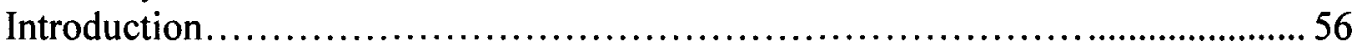

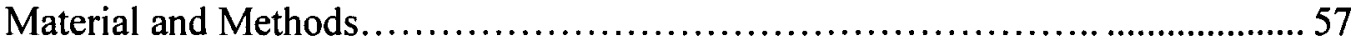

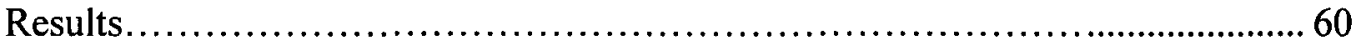

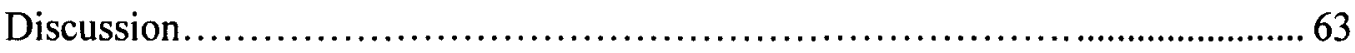

V. INTRASPECIFIC AND INTERSPECIFIC LARVAL COMPETITION BETWEEN

THE DENGUE VECTORS AE. AEGYPTI AND AE. ALBOPICTUS.....................65

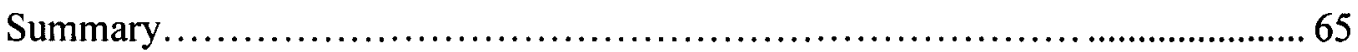

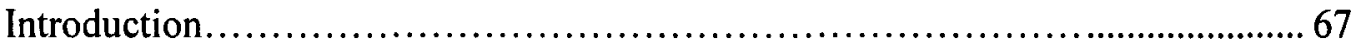

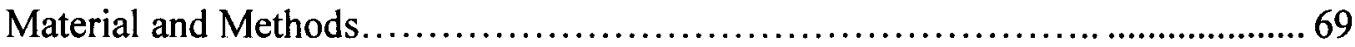

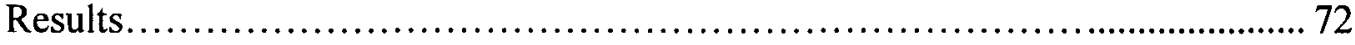

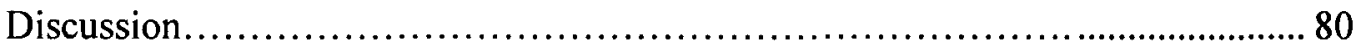

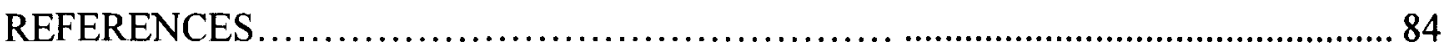

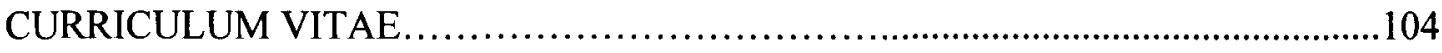




\section{LIST OF TABLES}

PAGE

1. Number of mosquitoes and pools by dengue serotype and mosquito species......29

2. Average post-exposure development time and exposure titer statistics. .30

3. Mixed model logistic regression for larval infection rate .46

4. Mixed model ANOVA for larval mortality rate. .47

5. Mixed model ANOVA for larval development time .49

6. Mixed model ANOVA for larval cephalothorax length.....................................50

7. Competition type mixed model ANOVA for larval cephalothorax length.............73

8. Competition type mixed model ANOVA for larval development time.............. 74

9. Competition type mixed model logistic regression for larval survivorship. .76

10. Competition intensity mixed model ANOVA for cephalothorax length 77

11. Competition intensity mixed model ANOVA for larval development time. . .78

12. Competition intensity mixed model logistic regression for larval survivorship. 


\section{LIST OF FIGURES}

PAGE

1. Dengue virus genome and gene function......................................................2

2. Correlation between body size and adult life history traits............................16

3. Effect of lifespan on dengue transmission rates..................................... 17

4. Per capita infection rates of Ae. aegypti and Ae. albopictus larvae....................31

5. Titer of positive Ae. aegypti and Ae. albopictus pools...................................32

6. Infection rates of Ae. aegypti and Ae. albopictus exposed to dengue-2 2............47

7. Mortality rate of larvae exposed to dengue- 2 and control treatment.................48

8. Development time of infected and uninfected larvae....................................49

9. Cephalothorax length infected and uninfected larvae...................................51

10. Consumption of larval and pupal detritus.................................................61

11. Effect of larval detritus of Ae. aegypti on growth and development.................62

12. Effect of interspecific competition on larval cephalothorax length..................73

13. Effect of interspecific competition on larval development time.......................75

14. Effect of sex and resource input on larval development time ........................75

15. Larval survivorship in intraspecific and interspecific containers......................76

16. Effect of intraspecific competition intensity on larval cephalothorax

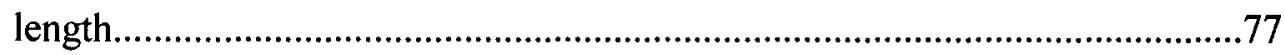

17. Effect of intraspecific competition intensity on larval development time.........78

18. Larval survivorship in low and high density intraspecific containers..............79 


\section{CHAPTER I}

\section{GENERAL INTRODUCTION}

\section{Dengue Virus and Mosquito Vectors}

Dengue virus (genus Flavivirus, family Flaviviridae) is currently the most serious arboviral threat in the world (Ooi and Gubler 2010, Guzman et al. 2010). More than 2.5 billion people in over 100 countries live in areas with endemic dengue transmission and up to $1 \%$ of the world's population may be infected every year resulting in over 10,000 deaths, yet just several hundred years ago it was exclusively a disease of non-human primates (Holmes and Twiddy 2003, Rico-Hesse 2007, Kyle and Harris 2008). Similar to previous global epidemics of yellow fever, typhus, and plague, the emergence of dengue has been facilitated by the spread and establishment of non-native arthropod vectors, ecological disruption, and the global movement of humans and commerce (Lounibos 2002). There is currently no treatment available for dengue; vaccine development has been complicated by the complex immune response to the virus and in the absence of an effective vaccine the only effective preventative measure is vector control (Whitehead et al. 2007, Zhang et al. 2010).

Dengue is a positive sense single-stranded enveloped RNA virus. The dengue genome is approximately $10.7 \mathrm{~kb}$ in length and is transcribed as a single open reading frame that encodes 3 structural (C, M, \& E) and 7 non-structural genes (NS1-NS5) (Gubler 1998). The 40-50 nm spherical virion consists of a genome-capsid protein 
complex surrounded by a lipid bilayer into which the membrane (M) and envelope (E) proteins are embedded. The viral non-structural proteins modulate the host immune response and perform functions not provided by the host cell such as viral replication, enzymatic processing of viral proteins, and virus assembly and maturation (Chambers et al. 1990).

The 5' and 3' non-coding regions have an important function in translation efficiency and in regulating viral replication (Leitmeyer et al. 1999, Guzman and Kouri 2002, Chaudry et al. 2007, Weaver and Vasilakis 2009).

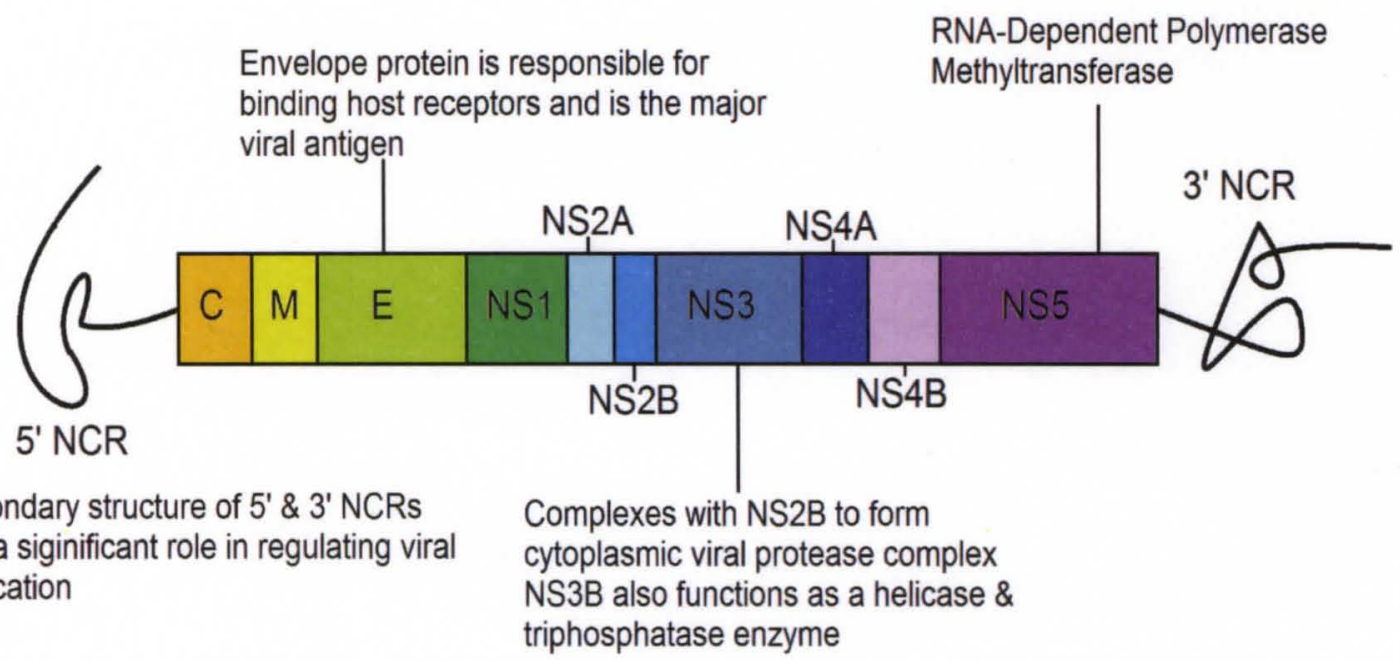

Figure 1. Dengue virus genome and gene functions.

There are four genetically distinct viral serotypes that comprise the dengue antigenic complex, and the genetic divergence between serotypes is often greater than divergence between different flavivirus "species" (Holmes and Burch 2000). Yet despite the substantial genetic diversity that exists between the dengue serotypes, all four serotypes cause nearly identical symptoms in humans and are vectored by the same mosquito species (Guzman et al. 2010). Within each serotype, strains that have less than a $6 \%$ sequence divergence based on the E/NS1 portion of the genome have been clustered 
into viral genotypes; there are five genotypes within dengue serotypes $1 \& 2$ and four genotypes within dengue serotypes $3 \& 4$ respectively (Rico-Hesse 1990).

Dengue is primarily transmitted to humans by Aedes aegypti and Aedes albopictus, though many other Aedes species such as Ae. mediovittatus, and the Ae. scutellaris group, which comprises 12 species are competent hosts for dengue (Frier and Rosen 1987, Frier and Rosen 1988). The prominent role that Ae. aegypti and Ae. albopictus have in dengue transmission can be primarily attributed to their adaptation to the peridomestic environment and their anthropophilic blood feeding tendencies (Harrington et al. 2001, Jansen and Beebe 2010). Dengue transmission to humans by mosquitoes involves complex interactions between mosquito genetics, viral genetics and environmental factors (Black et al. 2002). The environmental conditions in which the immature mosquito larvae develop have a particularly large influence on the viral susceptibility and transmission capability of adult females (Alto et al. 2008B).

\section{Dengue Emergence and Epidemiology}

The emergence of dengue as one of the most important human pathogens is directly associated with the spread and establishment of non-native mosquitoes that are competent hosts for dengue (Halstead 2007).

There are two ecologically and evolutionarily distinct transmission cycles of dengue; endemic dengue which is transmitted to humans by peridomestic Aedes species and sylvatic dengue which is transmitted between canopy dwelling arboreal Aedes species and non-human primates throughout peninsular Malaysia, and western Africa, primarily Senegal (Chen and Vasilakis 2011, Kyle and Harris 2008, Holmes and Twiddy 2003, Weaver and Vasilakis 2008, Weaver and Vasilakis 2009). Separate phylogenetic 
analyses of the NS5, E, and 3'NCR genome regions strongly support the conclusion that the sylvatic lineage is ancestral to endemic dengue (Wang et al. 2000, Holmes and Twiddy 2003, Gaunt et al. 2001, Shurtleff 2001). The four endemic dengue serotypes emerged independently and repeatedly from sylvatic dengue in a series of divergence events that occurred over the last 1,500 to 100 years (Wang et al. 2000, Holmes and Twiddy 2003, Vasilaskis et al. 2007, Vasilaskis et al. 2011). There also seems to be a consensus among the phylogenetic studies regarding the order of serotype divergence; dengue- 4 emerged first followed by dengue- 2 , then dengue- 3 and lastly dengue- 1 (Holmes and Twiddy 2003).

The emergence of endemic dengue into human populations is likely a result of ecological rather than evolutionary factors because no genetic adaptation is necessary for sylvatic dengue- 2 to be transmitted by Ae. aegypti, and the symptoms of sylvatic dengue infection in humans are indistinguishable from those of endemic dengue (Vasilakis et al. 2007, Vasilakis et al. 2008, Weaver and Reisen 2010). The canopy dwelling arboreal species Ae. niveus and Ae. furcifer found in Malaysia and Senegal respectively are known to engage in opportunistic feeding behavior on human populations established near the edges of forests and are likely responsible for transmitting sylvatic dengue to human populations (Vasilakis and Weaver 2008, Vasilakis et al. 2008, Kyle and Harris 2008). Studies in Senegal and Malaysia have shown that human populations living in rural or forested environments come into regular contact with sylvatic dengue (Vasilakis et al. 2008, Vasilakis et al. 2011). Therefore endemic dengue probably emerged in rural areas where arboreal mosquito species engaging in opportunistic feeding transmitted sylvatic dengue to human populations where it was acquired and transmitted among humans by 
peridomestic vectors, most likely Ae. albopictus (Wang et al. 2000, Mackenzie et al. 2004, Diallo et al. 2008, Holmes and Twiddy 2003, Vasilakis et al. 2011).

The geographic origin of endemic dengue has been a source of speculation among researchers for over three decades, and based on current data it is difficult to conclusively determine if endemic dengue has an Asian or African origin (Wang et al. 2000, Gaunt et al. 2001, Vasilakis and Weaver 2008,). The evidence supporting an African origin is based on the circulation of several closely related mosquito-borne flaviviruses in Africa and the presence of Ae. aegypti (Gaunt et al. 2001). However, the ancestral Ae. aegypti species, Ae. aegypti formosus is refractory to dengue and therefore the peridomestic vector necessary for transmission within human populations in Africa is missing (Weaver and Reisen 2010). The evidence supporting the Southeast Asian origin of endemic dengue is more convincing (Wang et al. 2000,Vasilakis and Weaver 2008, Vasilakis et al. 2011). Sylvatic strains from Asia have a deeper phylogenetic position relative to the African strains; all four sylvatic serotypes are found in Asian forests, and the peridomestic vector Ae. albopictus, which is often found in rural villages is competent for both sylvatic and endemic dengue (Wang et al. 2000, Moncayo et al. 2004 Vasilakis and Weaver 2008, Vasilakis et al. 2011).

Following the emergence of the four endemic serotypes into human populations either in Africa or Asia dengue was likely spread throughout the world by transoceanic shipping (Weaver and Vasilakis 2009, Weaver and Reisen 2010, Chen and Vasilakis 2011). Until recently, the geographic range of Ae. albopictus was limited to Asia; therefore it is likely that the spread of dengue was mediated by Ae. aegypti (Kyle and Harris 2008). Beginning in the $16^{\text {th }}$ century, domesticated Ae. aegypti spread from west 
Africa throughout the tropical regions of the world via slave and commerce ships. Ae. aegypti first traveled west to the Americas and then in the early $19^{\text {th }}$ century Ae. aegypti was brought east to Asia (Kyle and Harris 2008). Arbovirus transmission, initially yellow fever and then dengue, followed or was coincident with the spread of Ae. aegypti throughout the Americas (Paupy 2009). The spread of mosquito-borne disease following the geographic expansion of Ae. aegypti emphasizes the need to understand the vector ecology of mosquito species.

Clinical descriptions of a disease with similar symptoms to dengue date back to 992 A.D. in China, however it is difficult to verify whether these infections were indeed dengue (Gubler 1998, Kyle and Harris 2008, Chen and Vasilakis 2011). The similarity of dengue symptoms to symptoms of influenza, and of other arboviruses, particularly Chikungunya or yellow fever, and the predominance of asymptomatic cases make it difficult to determine the frequency of early endemic dengue transmission among human populations (Holmes and Twiddy 2003, Kyle and Harris 2008). Benjamin Rush recorded the first known thorough description of dengue's clinical symptoms during a 1781 epidemic in Philadelphia (Vasilakis and Weaver 2008). Until the middle of the $20^{\text {th }}$ century, the incidence of dengue transmission was sporadic, and epidemics were infrequent, occurring approximately once every 10-50 years (Gubler 1998).

The epidemiology of dengue has changed rapidly in the last 70 years. The onset of World War II brought about dramatic ecological and demographic disturbance in Southeast Asia, which created ideal conditions for the transmission of mosquito-borne diseases; the destruction of water infrastructure and war debris led to a significance increase in the density of Ae. aegypti (Gubler 1998, Mackenzie et al. 2004, Vasilakis and 
Weaver 2008). Large-scale military and refugee movements accelerated the dispersal of susceptible hosts, infected individuals, and mosquitoes throughout the region (Ooi and Gubler 2010). Not surprisingly, dengue transmission was widespread throughout Asia during the war (Kyle and Harris 2008). The world's first recorded dengue hemorrhagic fever (DHF) epidemic occurred in the Philippines in 1953, and since then the incidence of DHF/DSS (see below) has increased over 500-fold (Guzman and Isturiz 2010).

Postwar economic development fueled rapid unplanned urbanization in Southeast Asia that provided ideal conditions for viral transmission (Gubler 1998). Throughout the mid to late $20^{\text {th }}$ century, dengue spread from Southeast Asia throughout the world and now all four dengue serotypes are globally distributed (Guzman et al 2010). Before 1970 only 9 countries had experienced severe dengue epidemics, but now dengue is endemic in over 100 countries (Gubler 2004, Kyle and Harris 2008). Dengue epidemics are now occurring on a cyclical basis every 3 to 4 years in many countries and further increases in transmission are likely to occur as competent mosquito vectors continue to increase their geographic distribution, the global urbanization trend continues, and virulent genotypes emerge and spread (Gubler 2004).

\section{Mosquitoes as a Potential Reservoir Host for Dengue}

One of the great mysteries regarding the epidemiology of dengue is its persistence in the environment during periods unfavorable to transmission (Watts and Eldridge 1975, Rosen 1987, Lee and Rohani 2005, Vasilakis and Weaver 2008, Kyle and Harris 2008, Chen and Vasilakis 2010). Dengue is believed to persist in the environment since human infections are detected regularly even if there are long periods of low or zero incidence (Adams and Boots 2010). Given that there is no known vertebrate reservoir for dengue, 
one hypothesis for viral persistence in the environment during inter-epidemic periods is that mosquitoes may be a reservoir host for dengue (Joshi et al. 2002). Vertical transmission within mosquito populations may act as a mechanism to maintain dengue within mosquito populations during inter-epidemic periods that are unfavorable to horizontal transmission such as cool or dry conditions (Khin and Than 1983, Hull et al. 1984, Castro et al. 2004, Thenmozhi et al. 2007, Angel and Joshi 2008, Cecelio et al. 2009).

Vertical transmission in infected female Aedes may occur once dengue disseminates into the reproductive tract. Eggs become infected during fertilization as they move through the reproductive tract; dengue enters through a small pore on the anterior end of the egg known as the micropyle (Rosen 1987, Rosen 1988). Dengue is never vertically transmitted in the first egg batch following an infectious blood meal, but depending on the timing of the second blood meal dengue maybe transmitted to each subsequent egg batch (Rosen 1987). Ae. albopictus is more efficient at transmitting dengue vertically than Ae. aegypti (Bosio et al. 1992, Kow et al. 2001).

The number of mosquitoes in a population that are infected through vertical transmission is the product of the percentage of infected females that transmit dengue to their progeny (vertical transmission rate) and the number of larvae infected given that vertical transmission occurs (filial infection rate). Not all infected females will transmit dengue to their progeny; either because the virus is unable to disseminate into the reproductive tract, or as a result of death prior to the oviposition of infected eggs. There has only been one experimental study that thoroughly measured the vertical transmission rate in Ae. aegypti and Ae. albopictus females the vertical transmission rate in Ae. 
albopictus populations ranged between $11-41 \%$ and was 3\% in Ae. aegypti (Bosio et al. 1992)

When vertical transmission does occur the percentage of larvae that are infected is highly variable the filial infection rate can range from less than $1 \%$ to over $20 \%$ (Khin and Than 1983, Bosio et al. 1992, Mourya et al. 2001, Castro et al. 2004). Variability in the filial infection rate might be caused by viral replication within the egg; there is evidence to support the correlation between an extended egg incubation period with an increased filial infection rate (Mourya et al. 2001, Guo et al. 2007). Given the lack of studies investigating the vertical transmission rate and the extensive variability among filial infection rates, estimating the percentage of mosquitoes infected through vertical transmission has been difficult. A field surveillance study in Malaysia found that $1.33 \%$ of male Ae. aegypti and $2.15 \%$ of male Ae. albopictus mosquitoes tested positive for dengue. Assuming that males and females are infected through vertical transmission at a similar rate, then a vertical transmission rate of approximately $2.6 \% \& 4.3 \%$ for $A e$. aegypti and Ae. albopictus populations respectively could provide some indication of the vertical transmission rate in natural populations (Kow et al. 2001).

The broad range of reported vertical transmission rates has complicated the understanding of the role vertical transmission has in dengue epidemiology. Depending on the assumed vertical transmission rate, mathematical models have yielded conflicting conclusions as to whether vertical transmission could support the long-term persistence of dengue in the environment during inter-epidemic periods (Esteva and Vargas 2000, Adams and Boots 2010). However, Adams and Boots (2010) concluded that even low vertical transmission rates $(1-4 \%)$ can lead to short-term persistence, and that vertical 
transmission can intensify dengue epidemics by increasing the speed and intensity of viral transmission. Therefore, while more research needs to be done to clarify the role of vertical transmission in dengue epidemiology, it does appear to be significant.

One important aspect regarding the role of mosquitoes as a reservoir host in dengue epidemiology that is not known is the affect of dengue infection on larval development and survivorship. If there is a fitness cost to viral infection in larvae, then vertically infected larvae may not significantly contribute to dengue transmission as adults regardless of the vertical transmission rate. Understanding the effect of viral replication on larval development and survivorship is important to the development of accurate models regarding the role of vertically transmission in dengue epidemiology.

\section{Dengue Transmission and Pathogenesis}

Female mosquitoes may become infected with dengue after taking a blood meal from a viremic individual. After ingestion, the first stage of infection is penetration and replication within the midgut epithelial cells (Kramer and Ebel 2003). Dissemination from the midgut is the major barrier to transmission; the midgut, salivary glands, and ovaries, are all surrounded by a basement membrane that can prohibit the movement of viruses from those tissues (Hardy et al. 1983). Following dissemination into the hemocoel, dengue must then successfully replicate within the salivary glands before it can be transmitted to humans (Hardy et al. 1983, Kramer and Ebel 2003). The duration from the acquisition of an infectious blood meal until viral transmission is possible is known as the extrinsic incubation period (EIP) (Salazar et al. 2007). The EIP is significantly influenced by temperature and the infecting viral strain and typically ranges between 7-14 days (Anderson and Rico-Hesse 2006, Halstead 2008). 
Once infected with dengue, mosquitoes remain infectious for life. As a result infection may influence life history traits in mosquitoes. Yet little is known about the fitness effect of dengue infection in mosquitoes (Lambrechts and Scott 2009). Maciel-deFreitas (2011) experimentally infected adult Ae. aegypti females with dengue serotype-2 and found that infected mosquitoes had a significant decrease in life span and fertility, however these virus-induced effects were only seen in mosquito populations 14-21 days after infection (Maciel de Freitas et al. 2011). Given the high estimated mortality rate of mosquito populations in nature, the biological relevance of these results is not clear. Laboratory studies of other vector-virus combinations also found that viral induced mortality did not occur until roughly 21 days after infection. However, these studies found a significant decrease in fertility among infected females beginning with the first egg batch following infection (Scott and Lorenz 1998, Styer et al. 2007, Mamood et al. 2004). The reduction in fertility indicates that the cost of viral infection in mosquitoes maybe mediated through a diversion of resources rather than a physical destruction of mosquito tissue. Understanding the fitness cost of dengue infection in mosquitoes has important implications regarding viral evolution and transmission.

Following a bite from an infected mosquito, local immature Langerhans cells are most likely the first cell type to be infected with dengue (Clyde et al. 2006). Dengue infection disseminates throughout the lymphatic system as Langerhans cells migrate to lymph nodes where dengue infects monocytes and macrophages and then enters the blood stream (Whitehead et al. 2007, Martina et al. 2009). Acute febrile symptoms, if they occur, follow shortly after viremia and last 6-8 days. Humans typically have a high viral 
titer in their blood for about 5 days; during this viremic period mosquitoes may become infected with dengue while taking a blood meal (Whitehead et al. 2007).

There is a wide spectrum of clinical symptoms associated with dengue infection in humans, which are a result of the complex interactions between the infecting dengue strain, host immune response, age, and genetic background of the individual (McBride and Ohmann 2001, Mackenzie et al. 2004, Clyde et al. 2006). A majority of the estimated 100-million dengue infections each year are asymptomatic or subclinical (Chen and Vasilakis 2011). When clinical symptoms do occur, the most common outcome is classical dengue fever (DF), a self-limited, debilitating illness characterized by fever, headache, myalgia, arthralgia, retro-orbital pain, and rash (Gubler 1998, McBride and Ohmann 2001). Approximately $0.5 \%$ of dengue infections result in dengue hemorrhagic fever (DHF) or dengue shock syndrome (DSS) and require hospitalization. DHF/DSS is characterized by a sudden increase in vascular permeability, multiple bleeding abnormalities, thrombocytopenia, and can result in liver damage and death (Guzman and Kouri 2002, Halstead 2007). Infection with one serotype leads to immunity to that serotype only, while cross protection against other serotypes is transient and is lost after several months (Mackenzie et al. 2004) Secondary infection with a heterotypic serotype is associated with an increased risk of developing DHF/DSS. There is also an increasing risk of developing DHF/DSS as the duration between primary and secondary infection lengthens (Martina et al. 2009). 


\section{Role of the Larval Environment on Dengue Transmission}

As holometabolous insects, mosquitoes have a complex life cycle with an aquatic immature and a terrestrial adult stage that disperses to an ecologically distinct environment (Muturi et al. 2012). The biotic and abiotic conditions which larvae experience during development carry over into the adult stage and significantly influence both adult life history traits and vector competence for arboviruses (Klowden et al. 1988, Grimstad and Walker 1991, Nasci 1991, Alto et al. 2005, Styer et al. 2007, Alto et al. 2008 A \& B, Muturi et al. 2011, Muturi et al. 2012). Additionally interspecies interactions, which occur almost exclusively between larvae, can influence arboviral susceptibility, vectorial capacity, and the geographic distribution of mosquito species (Alto et al 2005, Bevins 2007, Reiskind and Lounibos 2009). A detailed understanding of how larval environmental conditions influence mosquito distribution and arbovirus transmission is critical to our understanding of vector borne disease.

\section{Mosquito Life Cycle}

Aedes females oviposit their eggs just above water level in a broad range of natural and artificial water filled containers (Wong et al. 2011). Embryonic development occurs within the egg and typically takes about 3 to 4 days, after which larvae will emerge once the eggs become submerged under water (Clements 1992). Eggs of both species are relatively desiccation resistant and can remain viable in the environment for weeks to months (Hawley 1988). Upon hatching, $1^{\text {st }}$ instar larvae emerge and begin to feed. Ae. aegypti and Ae. albopictus larvae are both classified as filtering-browsing feeders; larvae feed on fine particulate organic detritus and microbes throughout the water column and browse hard surfaces to resuspend and ingest fine particles (Merritt 
1992). As larvae develop they undergo 3 molts as larvae, and on their fourth molt will eclose as pupae (Clements 1992). The pupal stage is a resting non-feeding stage that is very active physiologically; certain larval organs such as the alimentary canal are broken down and replaced with adult organs generated from undifferentiated embryonic cells (Clements 1992). The pupal stage generally lasts about 48 hours, after which the adult mosquito will emerge (Zettel and Kaufman 2012). The length of the immature stage is highly dependent on temperature and the resources present within the larval habitat and there is a positive correlation between temperature and development time (Clements 1992). Newly emerged adults are initially dependent on the lipid and glycogen reserves they accumulated as larvae for survival (Briegel and Timmerman 2001).

Although both male and female adults may feed on nectar as a carbohydrate source, to initiate vitellogenesis female Aedes require a blood meal (Briegel et al. 2001). The blood feeding patterns of Ae. aegypti and Ae. albopictus have important epidemiological consequences. Ae. aegypti feeds almost exclusively on humans and is an extremely cautious feeder often requiring multiple blood meals for each gonotrophic cycle. The repeated contact between humans and Ae. aegypti is a contributing factor to its role as the vector of dengue epidemics (Gubler 1998). Ae. albopictus does display an anthropophillic host tendency but maintains a broad host range and may be a bridge vector between enzootic viruses and humans (Paupy et al. 2009). Adult males locate nearby females through acoustic signals and among Aedes mosquitoes mating occurs approximately $24-48$ hours after emergence and it generally occurs before the first blood meal (Takken et al. 2006). Oviposition will occur approximately $3-4$ days following a successful blood meal (Hawley 1988). 


\section{Effect of the Larval Environment on Adult Life History Traits}

Adult body size has a genetic basis in mosquitoes but is a very plastic trait that to a large extent is influenced by environmental conditions (Clements 1992). The resources that larvae are able to accumulate as they develop determines adult body size and has a substantial effect on life history traits such as life span, fecundity, immune function, sperm capacity and susceptibility to arboviruses (Clements 1992, Timmerman and Briegel 1996, Ponlawat and Harrington 2007, Alto et al. 2008A, Telang et al. 2011). Both biotic and abiotic factors within the larval environment influence development; temperature, resource inputs within the larval habitat, and competitive interactions between larvae all significantly influence adult body size (Clements 1992, Alto et al. 2005 Halstead 2007, Reiskind and Zarrabi 2012).

The effect of adult body size on dengue transmission is nuanced (Dodson et al. 2011) (Fig. 2). While small adults have a decreased life span and fecundity, arbovirus susceptibility and blood feeding rates are higher (Clements 1992, Scott et al. 2000, Briegel et al. 2001, Briegel and Timmerman 2001, Alto et al. 2008). The trade-off between size and transmission is not fully understood, but has important consequences (Muturi et al. 2012). Pupal cephalothorax length is highly correlated with adult size and is convenient approximation of adult fitness parameters (Koenraadt 2008). 


\begin{tabular}{|c|c|c|}
\hline Large Pupa & & Large Adult \\
\hline Small Pupa & & Small Adult \\
\hline Trait & Small Adult & Large Adult \\
\hline lifespan & $\checkmark$ & 人 \\
\hline fecundity & $\checkmark$ & $\checkmark$ \\
\hline $\begin{array}{c}\text { arbovirus } \\
\text { susceptibility }\end{array}$ & $\checkmark$ & $\checkmark$ \\
\hline sperm capacity & $\checkmark$ & $\checkmark$ \\
\hline $\begin{array}{c}\text { blood-feeding } \\
\text { rate }\end{array}$ & $\checkmark$ & $\checkmark$ \\
\hline
\end{tabular}

Figure 2. Correlation between body size and adult life history traits.

Mosquito life span is the most important determinant of a mosquito's ability to transmit pathogens, (Styer et al. 2007) (Figure 3). In order to transmit dengue horizontally to humans or vertically to their progeny, mosquitoes must survive the extrinsic incubation period and take a successful blood meal. Smaller adults have fewer nutrient reserves and under natural conditions have a shorter life span (Nasci 1986). The life span of field-caught mosquitoes has been estimated through mark-recapture studies and by the analyzing the reproductive history of captured females (Hawley 1988). Estimates based on reproductive history put the probability of daily survival between 0.71-0.88; mark-recapture data yielded estimates in the range of 0.61-0.85 for females, and $0.86-0.88$ for males (Hawley 1988). Small changes in the daily survival rate of mosquitoes can have a dramatic impact on the number of mosquitoes that survive the EIP and transmit dengue (Fig 3). 


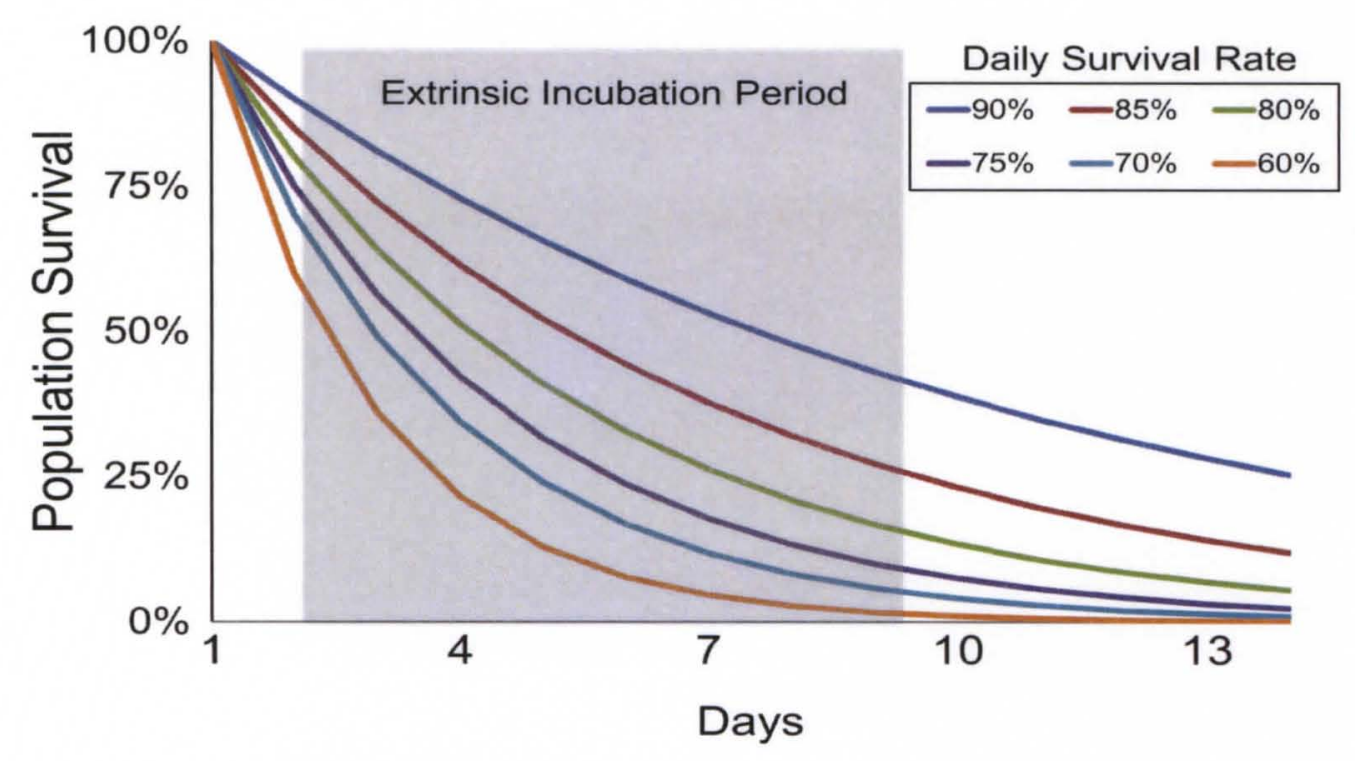

Figure 3. Effect of mosquito life span on dengue transmission rates.

Small Ae. aegypti and Ae. albopictus adults are significantly more susceptible to dengue virus than large adults (Alto et al 2008). The physiological mechanism underlying the increased susceptibility in smaller adults is not well understood. Grimstad and Walker (1991) demonstrated that small Aedes triseriatus were more susceptible to La Crosse virus than large mosquitoes and that small mosquitoes had a significantly thinner basement membrane lining the midgut, indicating that increased dissemination from the midgut may be responsible for the increased susceptibility in small larvae. Decreased immune function in small adults could also influence susceptibility: smaller adult Anopheles gambiae have a decreased ability to melanize beads, and environmental stress experienced by larvae influences the expression of anti-microbial peptides in adult $A e$. aegypti (Suwanchaichinda and Paskewitz 1998, Muturi et al. 2011)

Smaller Ae. aegypti also have a lower blood feeding success rate and need to take more blood meals per gonotrophic cycle relative to large adults. Increased host attack 
rates can significantly increase transmission rates, as dengue maybe transmitted during each attempted blood meal (Scott et al 2000).

The fecundity of female mosquitoes is established at the end of the larval stage, and is a function of body size (Kramer and Ebel 2003). The number of ovarioles is determined by the resources accumulated during the larval stage and governs the number of oocytes present at the start of each ovarian cycle; there is a strong positive correlation between female size and fecundity (Clements 1992). Furthermore, small adults typically have fewer nutrient reserves and need to divert more blood protein for their survival rather than oogenesis (Clements 1992). Body size significantly influences both sperm capacity as well as mating success. Large adults have significantly more spermatozoa and have an increased mating success rate, relative to smaller adults (Ponlawat and Harrington 2007).

\section{Effect of Larval Environment on Mosquito Distribution}

Within artificial containers, predation is generally low, resource inputs are usually limiting, and larval density is often high (Arrivillaga and Barrera 2004, Bevins 2007, Juliano 2009). Given the high density and limiting resources within larval habitats, resource competition is common among larvae (Alto et al. 2008). Multiple mosquito species often inhabit the same container and the interspecific competitive outcomes between species are often asymmetric and dependent on the resources present within the container (Yee 2008). When competition is highly asymmetrical, the competitive exclusion of species may occur, which can significantly influence the geographic distribution of the mosquito species (Juliano 1998, Juliano and Lounibos 2005). 
There are two primary resource inputs within artificial containers; animal detritus primarily in the form of invertebrate carcasses, and plant detritus, often leaf litter (Juliano 2009, Kaufman et al. 2010, Costanzo et al. 2011). As detritus decomposes nutrients are released into the larval habitat providing the substrate for the growth of microbial populations upon which larvae feed (Daughtery et al 2000, Yee et al. 2007, Pelz-Stelinski et al. 2010). Detritus types vary in their decay rates and in the amount of nutrients they release as they decay. Relative to leaf litter, animal detritus decomposes faster and releases more nutrients into the larval habitat (Yee and Juliano 2006, Pelz-Stelinski et al 2010). After 30 days in a larval free container, animal detritus lost $50 \%$ more mass than an equivalent amount of leaf litter (Yee and Juliano 2006). Decomposing animal detritus releases nitrogen and total reactive phosphorus into the larvae habitat and supports large increases in microbial growth (Yee and Juliano 2006). Not surprisingly, larval habitats containing invertebrate carcasses support higher mosquito population growth rates and produce larger adult mosquitoes compared to containers in which slower decaying detritus types such as leaf litter are present (Daughtery et al. 2000, Yee et al. 2007, Murrell and Juliano 2008). The presence of animal detritus within the larval habitat may reduce resource competition, and support the coexistence of different species, (Figures 2 \& 3)(Daughtery et al. 2000).

The outcome of interspecific interactions among larvae is often context dependent; competitive superiority is determined by the type and quantity of detritus present within the larval habitat (Juliano 2009). Ae. aegypti has been demonstrated to be the superior competitor relative to Ae. albopictus when artificial protein rich resources were utilized (Barrera 1996). However that competitive advantage was reversed when the 
primary resource input was plant detritus (Juliano 2009). The proximate mechanism for competitive superiority is not fully understood but may involve preferences for different resource types or differences in foraging behavior between species (Yee et al. 2004, Kesavaraju et al. 2007). When competitive interactions are highly asymmetrical, competitive exclusion can occur across the shared host range (Bevins 2007). The spread and establishment of Ae. aegypti and Ae. albopictus in non-native habitats has in part been mediated by their competitive superiority relative to native mosquito species, and has significantly influenced the epidemiology of dengue (Juliano and Lounibos 2005).

Following the arrival of Ae. aegypti in Southeast Asia in the $19^{\text {th }}$ century, Ae. aegypti displaced the native Ae. albopictus from the majority of the urban habitats that it inhabited (Hawley 1988, Kyle and Harris 2008). The establishment of Ae. aegypti in Southeast Asia is a major contributing factor to the frequent dengue epidemics and high disease burden in this region.

The geographic distribution of Ae. albopictus has rapidly expanded over the last 30 years; beginning in 1980, global shipping, primarily the used tire trade, spread $A e$. albopictus to Europe, South and North America and central Africa (Leisnham and Juliano 2009, Lambrechts et al. 2010). The introduction and subsequent spread of Ae. albopictus throughout the southeast United States has been associated with a dramatic decline in the geographic distribution and abundance of Ae. aegypti (Rey et al. 2006). Ae. albopictus has been shown to be a superior competitor to a number of native mosquito species throughout the U.S. and therefore it is likely to continue its range expansion throughout the Eastern and Midwestern U.S. (Bevins 2007, Kesavaraju et al. 2007, Costanzo et al. 2011). 


\section{Research Interests and Hypothesis}

In the course of their life cycle, mosquitoes undergo an ontogenetic niche shift; immature larvae and pupae development occurs within aquatic habitats from which adult mosquitoes emerge and disperse into the terrestrial environment. As discussed above, the aquatic larval stage has a important role in dengue transmission and epidemiology; the environmental conditions in which larvae develop determine adult life history traits and influence the geographic distribution of mosquito species. Through vertical transmission, larvae become infected with dengue and contribute viral persistence in the environment during periods unfavorable to transmission.

The broad objective of this dissertation is to increase our understanding of how the larval environment influences the role of larvae in dengue transmission. To achieve this, I conducted 5 experiments organized into 4 data chapters that address 4 specific unanswered biological questions.

1. Are Ae. aegypti and Ae. albopictus larvae susceptible to dengue virus, and could horizontal transmission occur within the larval habitat? (Chapter 2)

2. What is the fitness effect of dengue infection within Ae. aegypti and Ae. albopictus larvae? (Chapter 3)

3. How is larval detritus utilized within the habitat and what is its effect on larval development? (Chapter 4)

4. How does larval detritus influence the competitive interactions within the larval habitat? (Chapter 5) 
All experiments involving dengue virus were approved by the University of Louisville Institutional Review Board, \#07-070, "Dengue Virus Interactions with Aedes

Mosquitos". 


\section{CHAPTER II}

\section{SUSCEPTIBILITY OF LARVAL AEDES AEGYPTI AND AEDES ALBOPICTUS TO DENGUE VIRUS (DIPTERA: CULICIDAE).}

\section{SUMMARY}

Mosquitoes vertically transmit many arthropod borne viruses, and as a consequence arboviruses are often present within the larval environment. We tested the hypothesis that Aedes aegypti (Linnaeus) and Aedes albopictus (Skuse) larvae were susceptible to dengue virus through two infection methods: exposure to dengue in the larval growth environment via viral supernatant, and exposure to infected tissue culture along with viral supernatant. In addition to investigating for the first time the susceptibility of larval Ae. albopictus to dengue virus, we analyzed the infection rate and viral titer of infected pools of Ae. aegypti when exposed to multiple serotypes of dengue. We found that both Ae. aegypti and Ae. albopictus larvae were susceptible to the threedengue virus serotypes to which they were exposed regardless of the exposure method and that there were significant differences between the serotypes in infection titer and infection rate. The finding that larval Ae. aegypti and Ae. albopictus are susceptible to dengue indicates that dengue might be able to spread among larvae within the larval habitat potentially contributing to the persistence of dengue in the environment. 


\section{INTRODUCTION}

Dengue virus (genus Flavivirus, family Flaviviridae) is currently the most serious arboviral threat in the world. More than 2.5 billion people in over 100 countries live in areas with endemic dengue transmission and up to $1 \%$ of the world's population may be infected every year with one or more of the four dengue serotypes resulting in approximately 10,000 deaths (Kyle and Harris 2008, Guzman et al. 2010). Primary infection with dengue generally results in dengue fever, a painful self-limited febrile illness characterized by headache, fever, and rash. Disease manifestations with increased clinical severity require hospitalization and are dependent on the immune status of an individual and the infecting genotype of dengue (McBride and Bielefeldt-Ohmann 2000).

The two primary invertebrate hosts of endemic dengue are the peridomestic species Aedes aegypti (Linnaeus) and Aedes albopictus (Skuse) (Chen and Vasilakis 2011). Aedes aegypti, the primary vector of dengue is found in tropical and subtropical regions throughout the world and is well adapted to urban areas and the domestic environment (Wong et al. 2012). Ae. albopictus originated in Asia but is currently found throughout the western hemisphere, Europe, and Africa due to a dramatic increase in geographic range that began in the 1980's (Lambrechts et al. 2010). Ae. albopictus has been considered a secondary vector of dengue because it feeds on a broader range of vertebrates and has a less intimate association with humans, however, the role of $A e$. albopictus in a recent arboviral epidemic indicates that the vector potential of $A e$. albopictus is significant and should not be overlooked (Tsetsarkin et al. 2007, Paupy et al. 2009). 
In addition to the horizontal transmission of dengue to humans, dengue transmission also occurs within Ae. aegypti and Ae. albopictus populations (Rosen et al. 1983, Rosen 1987a \& b, Bosio 1992). The most thoroughly documented mechanism by which dengue is transmitted among mosquitoes is through vertical transmission from an infected female to her progeny (Freier and Rosen 1987, Bosio et al. 1992, Joshi et al. 2002, Cecílio 2009). When vertical transmission occurs, the percentage of infected progeny may exceed $10 \%$ and filial infection rates of $40-60 \%$ have been reported when eggs laid by an infected female were incubated for more than 1 month (Freier and Rosen 1987, Bosio et al. 1992, Mourya et al. 2001, de Castro et al. 2004). Increased filial infection rates after a prolonged incubation may be a consequence of dengue replication within the egg (Guo et al. 2007). Additionally, Ae. albopictus males are capable of unidirectional venereal transmission to uninfected females (Rosen 1987a); whether male Ae. aegypti are also capable of sexual transmission has yet to be investigated. Vertical and sexual transmission of dengue among mosquitoes is thought to contribute to the persistence of endemic dengue in the environment (Ibanez-Bernal et al. 1997, Joshi et al. 2002, Adams and Boots 2010).

Another potential mechanism for viral amplification within mosquito populations could occur among larvae (Miller et al. 1978, Turell et al. 1990). Susceptibility of Aedes mosquito larvae to arboviruses has been reported for Rift Valley Fever virus, La Crosse virus, Yellow Fever virus, Japanese B encephalitis virus, St. Louis encephalitis virus, and West Nile virus when larvae are placed into a viral suspension (Peleg 1965, Miller et al. 1978, Turell et al. 1990). Although an initial study investigating the susceptibility of larval Ae. aegypti species to dengue (Peleg 1965) reported that larvae were susceptible 
when exposed to infected dead chick embryos, that study did not thoroughly investigate infection rates, infection rate variability among serotypes, or the viral titer of infected mosquitoes. Here we report results of experiments in which the susceptibility of larval Ae. aegypti and Ae. albopictus to dengue was assessed. We tested larval susceptibility to three viral strains representing three of the four dengue serotypes, and did so using two possible infection routes: exposure to free virus in the larval growth environment via viral supernatant, and exposure to infected tissue culture along with viral supernatant. In addition to investigating for the first time the susceptibility of larval Ae. albopictus to dengue, we analyzed the infection rate and viral titer of infected pools of Ae. aegypti when exposed to multiple serotypes of dengue. We found that Ae. aegypti and Ae. albopictus larvae were susceptible to dengue virus serotypes 2, 3, and 4 and that there were significant differences between the serotypes in viral titer and infection rate.

\section{MATERIALS and METHODS}

Mosquitoes, Virus, and Cell Culture. The Ae. aegypti colonies used in this study were established from adults collected in Palm Beach County, Florida, and had been colonized

for less than 1 year. The Ae. albopictus colony was established from adults that were field caught in Louisville, Kentucky and had been in the laboratory for less than 1 year. Mosquito populations were maintained at $30^{\circ} \mathrm{C}+/-1.0,70 \% \mathrm{RH}$ with a $14: 10 \mathrm{~L}: \mathrm{D}$ photoperiod. Adult mosquitoes were fed regularly on pig's blood and were given cotton swabs soaked in a $10 \%$ sugar solution. After hatching, mosquito larvae were reared in reverse osmosis water and were provided with Tetra-min fish food. Dengue-2 strain D000137, dengue- 3 H-87, and dengue-4 P-84 were kindly provided by Dr. Kathy Hanley 
(New Mexico State University) and propagated in African green monkey kidney cells

(Vero). These Vero cells were maintained at $37^{\circ} \mathrm{C}$ in minimum essential medium (MEM) supplemented with 10\% fetal bovine serum (FBS), $2 \mathrm{mM}$ L-Glutamine and 0.10 $\mathrm{mg} / \mathrm{ml}$ gentamicin.

Experimental Exposure of Larvae to Dengue. To determine whether larvae were susceptible to infection through exposure to free virions in MEM, $2^{\text {nd }}$ instar Ae. aegypti and Ae. albopictus larvae were placed into wells of a 24-well plate containing dengue virus supernatant that was collected 5 days post inoculation from Vero cells inoculated at a multiplicity of infection of 0.1 . Every 24 hours for 4 days larvae were removed from 24 well plates, rinsed twice in $150 \mathrm{ml}$ of reverse osmosis water, and placed into a fresh virus pool. After a 4-day exposure period, larvae were rinsed twice in $150 \mathrm{ml}$ of distilled water and were reared to pupation in containers with $150 \mathrm{ml}$ reverse osmosis water and tetramin fish food at $28^{\circ} \mathrm{C}$. No pupae emerged during the exposure period. Larvae were also exposed to infected cell culture plus viral supernatant to determine if ingestion of infected cells was necessary to develop infection. Larvae exposed to infected cell culture plus supernatant were exposed in an identical method except that the wells they were placed into contained an infected monolayer of Vero cells. Immediately before larvae were exposed to dengue a $150 \mu \mathrm{l}$ aliquot of supernatant was taken for the determination of exposure titer for both exposure methods. For a small subset of assays, another $150 \mu \mathrm{l}$ aliquot was taken 24 hours later to determine the decrease in exposure titer. Control larvae were exposed to virus-free MEM or virus-free MEM plus uninfected cell culture in an identical manner. In a previous experiment we found that larvae were able to successfully develop in supernatant harvested from uninfected cells (data not shown). 
Assay for Infection Outcome. Upon pupation, pupae were rinsed in distilled water and pooled into groups of 3-5 mosquitoes. Each pool was then homogenized in MEM (Hyclone) with antibiotics by manual grinding in a $1.5 \mathrm{ml}$ micro centrifuge tube with a polycarbonate pestle (USA Scientific); filtered through a $.22 \mu \mathrm{M}$ pore filter $(13 \mathrm{~mm}$, Fisher PVDF); and the filtrate was frozen at $-80^{\circ} \mathrm{C}$ for later assay of virus titer. Some pools contained $3^{\text {rd }}$ or $4^{\text {th }}$ instar larvae along with pupae. To determine if the mosquito pools were infected with dengue, the filtrate was then serially diluted and used to inoculate confluent Vero monolayers in 24-well plates. These cells were then incubated at $37^{\circ} \mathrm{C}$ for 2 hours, and overlaid with $0.8 \%$ methylcellulose (Acros Organics) dissolved in MEM supplemented with 2\% FBS 2mM L-glutamine (Hyclone) and $0.10 \mathrm{mg} / \mathrm{ml}$ gentamicin. Thereafter they were incubated for 5 days and then the monolayers were immunostained (KPL). Viral titers in infected pools were recorded as $\log 10$ focus forming units per $\mathrm{ml}(\mathrm{ffu} / \mathrm{ml})$.

Statistical Analysis. The estimated number of mosquitoes that became infected after dengue virus exposure was calculated with pools of variable size using the method of C.T. Le (Le 1981). Additionally, the minimum and maximum infection rates were also determined by calculating the minimum and maximum number of mosquitoes that could be infected given the number of positive pools. For each species and serotype combination, the maximum infection rate is calculated by assuming that every mosquito from a positive pool is infected (\# of mosquitoes within positive pools / \# of mosquitoes in each treatment). The minimum infection rate was calculated by assuming that only one mosquito in each positive pool was infected (\# of positive pools / \# of mosquitoes in each treatment). We assessed the dependence of the binomial response variable pool infection 
status on the fixed predicator variables serotype, exposure method and their interaction using SAS PROC GLIMMIX (SAS 9.3). Using the reduced dataset of only dengue positive pools, we then assessed the effect of the fixed predictor variables serotype, exposure method and their interaction on the viral titer of infected pools using SAS PROC GLM. Significant main effects were followed by pairwise contrasts of means (Bonferroni adjustment for multiple comparisons, SAS 9.3). The titer data from pools were log-transformed prior to statistical analysis to improve normality.

\section{RESULTS}

A total of 540 Ae. aegypti and 164 Ae. albopictus in pools of 3 to 5 mosquitoes were assayed to determine susceptibility of larvae to dengue-2, 3 and 4 (Table 1). We found that both mosquito species were susceptible to all three serotypes of dengue virus (Table 1, Figs 4A, B). Exposing Ae. aegypti larvae to either viral supernatant or viral supernatant and infected cells in the growth environment did not result in a difference in the proportion of pools that were infected $(\mathrm{F}=0.19 \mathrm{df}=1 \mathrm{P}=0.66)$, nor was there a significant serotype by exposure method interaction $(\mathrm{F}=0.06 \mathrm{df}=2 \mathrm{P}=0.94)$. Overall the estimated percentage of larvae infected ranged from 4 to $23 \%$, and there were significant differences in the susceptibility of Ae. aegypti to the 3 dengue serotypes (Fig 4A). None of the control larvae were infected (data not shown).

Table 1. Number of mosquitoes and pools by dengue serotype and mosquito species.

\begin{tabular}{|clcccc|}
\hline Serotype & Species & $\begin{array}{c}\text { Total \# } \\
\text { mosquitoes }\end{array}$ & $\begin{array}{c}\text { Total } \\
\text { pools }\end{array}$ & Positive pools & $\begin{array}{c}\text { Percentage of } \\
\text { pools positive }\end{array}$ \\
\hline \multirow{2}{*}{ Dengue-2 } & Ae. aegypti & 105 & 26 & 17 & $65 \%$ \\
& Ae. albopictus & 85 & 22 & 7 & $32 \%$ \\
\hline \multirow{2}{*}{ Dengue-3 } & Ae. aegypti & 220 & 52 & 21 & $40 \%$ \\
& Ae. albopictus & 32 & 8 & 1 & $13 \%$ \\
\hline \multirow{2}{*}{ Dengue-4 } & Ae. aegypti & 237 & 61 & 11 & $18 \%$ \\
& Ae. albopictus & 40 & 10 & 5 & $50 \%$ \\
\hline & Ae. aegypti total & 562 & 139 & 49 & $35 \%$ \\
& Ae. albopictus total & 157 & 40 & 13 & $33 \%$ \\
& Overall total & 719 & 179 & 62 & $35 \%$ \\
\hline
\end{tabular}


Exposure titer aliquots taken at the end of a 24 hour exposure period found that exposure titer decreased approximately 10 -fold for all 3 serotypes (data not shown).

After the 4-day exposure to dengue, larval development time within secondary containers averaged about 7 days, which provided a sufficient incubation period for dengue virus replication within larvae. The average development time and exposure titer for each species-serotype combination are provided in table 2 .

Table 2. Average post-exposure development time and exposure titer statistics.

\begin{tabular}{|llccl|}
\hline Serotype & Species & $\begin{array}{c}\text { Average post-exposure } \\
\text { development time (std dev) }\end{array}$ & $\begin{array}{c}\text { Average exposure titer } \\
\text { (std dev) }\end{array}$ & Exposure titer range \\
\hline \multirow{2}{*}{ Dengue-2 } & Ae. aegypti & $7.7(1.74)$ & $8.5 \times 10^{5}\left(3.4 \times 10^{5}\right)$ & $4.7 \times 10^{5}-1.9 \times 10^{6}$ \\
& Ae. albopictus & $9.1(1.86)$ & $4.3 \times 10^{5}\left(4 \times 10^{5}\right)$ & $3.3 \times 10^{4}-1.4 \times 10^{6}$ \\
\hline \multirow{2}{*}{ Dengue-3 } & Ae. aegypti & $8.5(2.2)$ & $8.9 \times 10^{5}\left(5.1 \times 10^{5}\right)$ & $2 \times 10^{5}-1.8 \times 10^{6}$ \\
& Ae. albopictus & $9.4(1.4)$ & $7.1 \times 10^{5}\left(6.3 \times 10^{5}\right)$ & $3.4 \times 10^{4}-1.5 \times 10^{6}$ \\
\hline \multirow{2}{*}{ Dengue-4 } & Ae. aegypti & $7.9(1.4)$ & $6.3 \times 10^{5}\left(3.9 \times 10^{5}\right)$ & $3.5 \times 10^{4}-1.4 \times 10^{6}$ \\
& Ae. albopictus & $7.9(2.2)$ & $1.1 \times 10^{6}\left(3.8 \times 10^{5}\right)$ & $8 \times 10^{5}-1.7 \times 10^{6}$ \\
\hline
\end{tabular}

For Ae. aegypti, we also quantified the titer of dengue positive pools (Fig. 5A). Interestingly, larvae became infected with dengue after exposure to titers $\left(3.3 \times 10^{4}\right.$ $\mathrm{ffu} / \mathrm{ml}$ ) that were lower than the titers observed in a number of positive pools. In several cases, the titer of positive pools was greater than the titer to which they were exposed (Fig 5). As was true regarding the infection rates, the titer of infected pools did not differ significantly between exposure routes $(\mathrm{F}=0.10 \mathrm{df}=1 \mathrm{P}=0.74)$, nor was there any exposure method by serotype interaction $(\mathrm{F}-1.04 \mathrm{df}=2 \mathrm{P}=0.36)$. We therefore combined the $A e$. aegypti data from the two exposure methods for statistical comparisons between serotypes. In Ae. aegypti the probability of detecting virus in a pool of pupae differed significantly among serotypes $(\mathrm{F}=3.96 \mathrm{df}=2 \mathrm{P}<0.02)$. This pattern is driven due to 
significantly higher infection probabilities following dengue- 2 exposure than following exposure to dengue-4 $(\mathrm{F}=2.80 \mathrm{df}=120 \mathrm{P}<0.01)$ (Fig 4A).

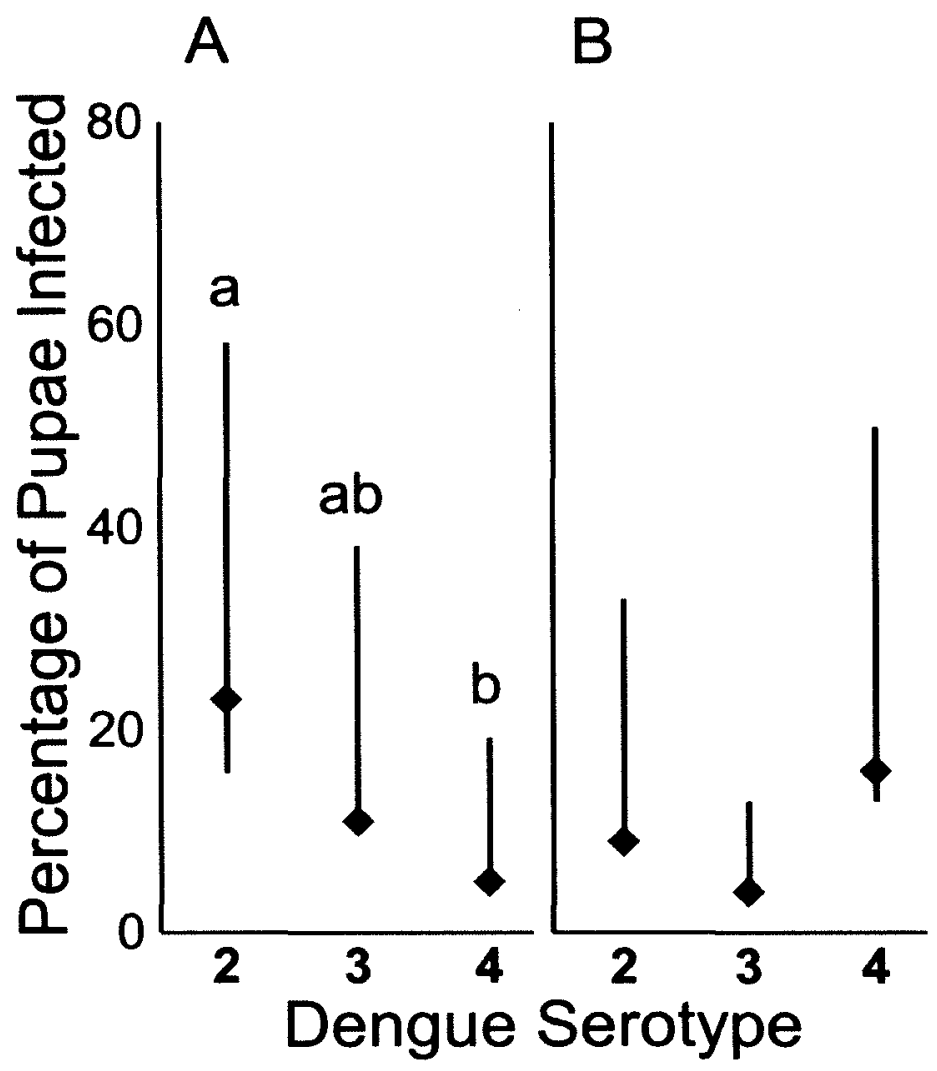

Figure 4. Per capita infection rates. Minimum-maximum infection range (black line) and estimated infection rate (diamond marker) of (A) Ae. aegypti and (B) Ae. albopictus larvae exposed to dengue-2, 3 and 4 . The letters over the estimates indicate the results of pairwise comparisons of serotypes from a logistic regression with serotype, exposure method and their interaction as predictor variables, and recovery of virus in the test pool as the response. Each letter that appears multiple times designates pairs of serotypes that did not differ significantly at $\mathrm{p}=0.05$, controlling for an overall alpha across multiple comparisons of 0.05 . Exposure methods did not differ significantly.

Among Ae. aegypti pools that were positive for dengue, we detected a significant overall difference among serotypes in mean titer that was driven by dengue-2. Larvae exposed to dengue- 2 had a significantly higher viral titer relative to the dengue-3 exposed larvae $(\mathrm{F}=5.76 \mathrm{df}=1 \mathrm{P}<0.01)$. Dengue-4 exposed larvae also had a significantly higher pool titer relative to dengue- $3(\mathrm{~F}=4.19 \mathrm{df}=1 \mathrm{P}<0.04)$ though this contrast was not significant after adjusting for multiple comparisons. The differences in mean pool titer between dengue- 2 and dengue- 4 were not significant $(\mathrm{F}=2.12, \mathrm{df}=1 \mathrm{P}<0.15)($ Fig. $5 \mathrm{~A})$. 
Although sample size limitations preclude statistical analyses in Ae. albopictus,

differences among serotypes in rates of larval infection do not appear to be consistent across mosquito species. Whereas dengue-2 exposure yielded a higher proportion of infected pools than dengue- 3 in both species, the relative rates of infection for dengue-3 $\& 4$ differed between the two species (Table 1, Fig. 4).

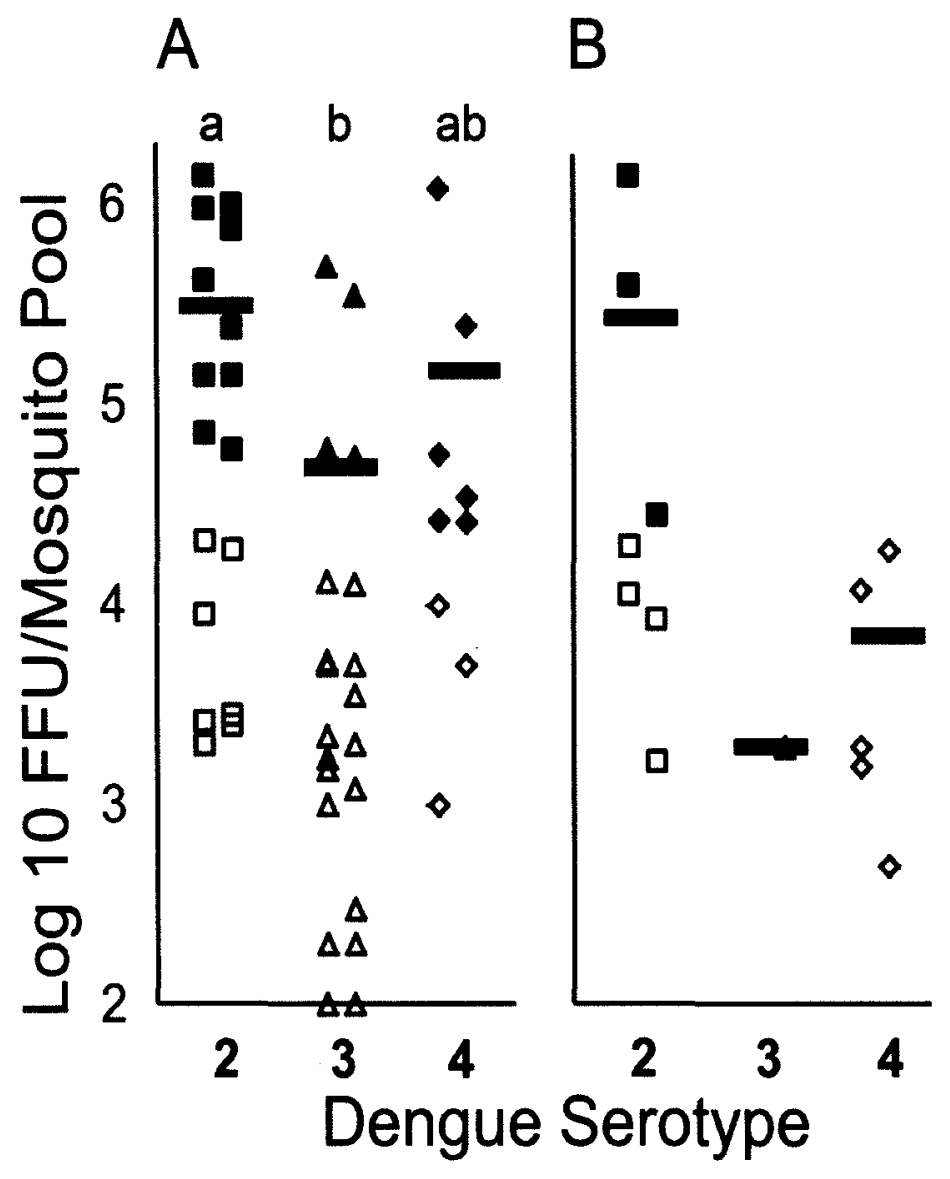

Figure 5. Titer of positive $\boldsymbol{A}$ e. aegypti and $\boldsymbol{A}$. albopictus pools. Overall average exposure titer (black bar) for each serotype, and titer of each positive pool from (A) Ae. aegypti and (B) Ae. albopictus larvae infected with dengue-2, 3, and 4. Black markers indicate pools that had a higher titer then the exposure titer to which they were exposed. Gray markers indicate pools with a higher titer than the minimum exposure titer to which larvae were susceptible. The letters over the estimates indicate the results of pairwise comparisons of serotypes from a general linear model with serotype, exposure method and their interaction as predictor variables, and pool titer as the response variable. Each letter that appears multiple times designates pairs of serotypes that did not differ significantly at $p=0.05$. Exposure methods did not differ significantly. 


\section{DISCUSSION}

Larvae were exposed to dengue virus for 4 days, rinsed thoroughly, and then transferred to secondary containers to complete their development in distilled water; larval development continued for an average of about 7 days (range from 6-14 days) after dengue exposure (Table 2). It is likely that there was a small quantity of virus carried over into the developmental containers either attached to the surface of the larvae or free virions carried over from the exposure supernatant. Given the significant dilution of exposure media during the two larval rinses and the decrease of dengue virus infectivity in solution it is extremely unlikely that any dengue incidentally carried over into the developmental containers would remain viable throughout the duration of larval development (Sithisarn et al. 2003). Similarly, within the developmental containers any dengue attached to the exoskeleton would be exposed to water and over time would become uninfectious, and dengue attached to the exoskeleton would be shed during molting as larvae developed. Most of the microflora within the midgut is expelled prior to pupation during the final defecation of $4^{\text {th }}$ instar larvae (Moll et al. 2001). While solid material remains within the midgut of the pupa following the larval-pupal molt (data not shown), this is unlikely to account for the observed dengue in pools of pupae as the high titers observed in the pools strongly supports viral replication (Fig 5). Furthermore, the midgut epithelium undergoes histolysis during the pupal stage removing a major barrier for viral entry, so that any virus present in the midgut at pupation will have access to the pupal tissues. Similarly, the foregut and hindgut are lined with cuticle that is shed with the exoskeleton during the molt. Because of this, it is unlikely that dengue detected in pupal 
pools represents virus within the gut or attached to the cuticle of pupae that remain uninfected. Therefore we believe that the potential for false positives due to environmental contamination of uninfected pupae is very low in our study.

Within the larval habitat, larvae would most likely encounter dengue through the consumption of infected mosquito carcasses. Invertebrate carcasses are a nutrient-dense resource and larvae are attracted to and consume dead invertebrate carcasses (Daugherty et al. 2000, Yee et al. 2007). We have found that dead larvae will often be completely consumed by other larvae within 24 hours (data not shown). Though dengue, like other arboviruses, does not remain infectious in the environment between hosts for more than several days, the rapid scavenging of carcasses ensures that larvae will come into contact with dengue when infected mosquitoes die within the larval habitat (Miller et al. 1978, Turell et al. 1990). When infected larvae or adults die in the larval habitat, their carcasses are a potential source of infection to the larvae that consume them.

While the dengue titers in larvae infected through vertical transmission are not known, the titer of infected adults can be above $1 \times 10^{6} \mathrm{ffu} / \mathrm{ml}$ (Salazar et al. 2007). We found that larvae were infected with dengue virus after exposure to titers as low as $3.3 \mathrm{x}$ $10^{4} \mathrm{ffu} / \mathrm{ml}$, indicating that the viral threshold to larval infection is relatively low. Larvae infected with dengue may have increased mortality rates (Joshi et al. 2002). Therefore, it may not be uncommon for larvae to come into contact with infected carcasses. Dengue is capable of replicating to high titer in larvae. Given that the dengue virus titer within positive pools was greater than that necessary to infect larvae, our results indicate that infected larvae may be infectious when consumed (Fig 5). Future research is necessary to determine the titer of larvae infected through vertical transmission in natural populations. 
Overall, we found that Ae. aegypti larvae exposed to dengue-2 had a significantly higher probability of becoming infected than those exposed to dengue-4. Dengue-2 infected Ae. aegypti pools had a significantly higher titer than those infected with dengue-3 (Fig 5A). The dengue-2 used in this experiment is a Southeast Asian (SEA) genotype. The SEA genotype has a number of consensus mutations that result in efficient replication and the SEA genotype has been shown to replicate to higher titer than dengue-2 American (AM) genotype in both adult Ae. aegypti and human monocytes (Cologna and Rico-Hesse 2005, Chaudhry et al. 2006). Ae. aegypti is also more susceptible to the SEA genotype than the AM genotype and as a consequence the SEA genotype has competitively displaced the AM genotype throughout much of the western hemisphere over the last thirty years (Armstrong and Rico-Hesse 2003, San Martin et al. 2010). The higher probability of infection and titer associated with the SEA genotype in this study suggests that this virus' replication advantage extends to larval Ae. aegypti mosquitoes as well.

Dengue virus transmission, known to occur between mosquitoes by both vertical and sexual transmission, is believed to facilitate the persistence of dengue in the environment during periods unfavorable for transmission to humans (Rosen 1987a \& b), de Castro et al. 2004, Angel and Joshi 2008, Cecilio et al. 2009). While vertical and sexual transmission do not occur at rates high enough to support long term persistence in the environment, vertical transmission may contribute to the temporary persistence of dengue in the environment and play an important role in dengue epidemics (Kuno 1995, Adams and Boots 2010). Given the susceptibility of Ae. aegypti and Ae albopictus larvae to dengue virus (Table 1, Fig 4), even at relatively low titers, the increased mortality of 
infected larvae (Joshi et al. 2002) and consumption of dead larvae within larval habitats (unpublished observation), horizontal transmission of dengue within larval populations could occur. Horizontal transmission of dengue could maintain or even increase infection rates in larval populations and therefore increase the rate of infected adults emerging from the larval habitat, potentially contributing to the persistence of dengue in the environment in the absence of amplification in human hosts. Therefore, further study of the interaction between dengue virus and larval mosquitoes, including the potential of horizontal transmission among larvae in the ecology of this important emerging disease, is warranted. 


\section{CHAPTER III}

\section{FITNESS COST OF DENGUE VIRUS INFECTION IN AEDES AEGYPTI AND AEDES ALBOPICTUS LARVAE (DIPTERA: CULICIDAE)}

\section{SUMMARY}

Female mosquitoes vertically transmit dengue to their progeny, but how infection influences larval growth, development, and survivorship is poorly understood. Determining the effect of dengue replication in vertically infected larvae is important because it could significantly influence the ability of mosquitoes infected as larvae to transmit dengue to humans and within mosquito populations. Understanding the effect of dengue infection in larvae would also provide a valuable insight regarding the role that vertically infected mosquitoes have in dengue epidemiology and could improve the epidemiological modeling of dengue. In this experiment, we tested the hypothesis that viral infection reduces fitness in Ae. aegypti and Ae. albopictus larvae using two epidemiologically relevant dengue-2 genotypes. Additionally, after larval exposure to dengue, larvae were reared at two different nutrient levels to determine if resources present within the larval habitat influence the effect of dengue infection.

We found that the effect of dengue infection within larvae was sex-specific; infected male larvae had a significantly longer development time and had a reduced cephalothorax length relative to uninfected males while infected females were not significantly different from uninfected females in either developmental time or 
cephalothorax length. Among infected larvae, there was no difference between the Southeast Asian and American genotypes in either developmental time or cephalothorax length. Overall, there was a trend of higher mortality in dengue-exposed Ae. albopictus but not in Ae. aegypti larvae, though it was not statistically significant. The amount of resource inputs that larvae were given influenced neither infection rate nor the development time of infected larvae, but did influence the cephalothorax length among infected larvae, providing limited support for the hypothesis that resource levels can influence the effect of dengue infection within mosquitoes. Our data indicate that female mosquitoes infected through vertical transmission will not have increased mortality rates or a decreased life span as a result of infection and will likely to able to transmit dengue to humans. In this experiment, we investigate for the first time the effect of dengue replication within male mosquitoes. Dengue infection in male mosquitoes significantly increased development time and reduced cephalothorax length, indicating that the ability of infected males to successfully mate with females may be diminished relative to uninfected males. The detected effect of infection in male mosquitoes could influence dengue transmission in two ways: indirectly through alterations in mosquito population dynamics, and directly through the sexual transmission of dengue to female mosquitoes.

\section{INTRODUCTION}

A major unanswered question in the study of vector-borne disease is whether vector-borne pathogens decrease the fitness of their invertebrate hosts (Ferguson and Read 2002, Elliot et al. 2003). The historical assumption regarding the virulence of vector-borne pathogens is that they should evolve towards a benign relationship with 
their vectors since they are dependent upon vector survival for their dispersal (Levin 1996, Lambrechts and Scott 2009). This paradigm, known as "common sense" was challenged on theoretical grounds by Anderson \& May (1982) who argued that selection could favor the evolution and maintenance of virulence if it led to increased pathogen transmission (Alizon et al. 2008). Recent empirical research has also challenged the paradigm that vector-borne pathogens should evolve towards commensalism within their invertebrate hosts (Styer et al. 2007, Reiskind et al. 2010). However, though both empirical and theoretical arguments have challenged the "common sense" paradigm, it is not currently well understood what effect vector-borne pathogens such as dengue virus have on the fitness of their invertebrate hosts (Lambrechts and Scott 2009).

Understanding the effects of viral pathogens on their invertebrate hosts will allow us to determine how and to what degree the latter can serve as reservoir hosts for arboviruses during periods that are unfavorable for transmission. Viral persistence in the environment during periods unfavorable to transmission is believed to be maintained primarily through vertical transmission among invertebrate hosts that vector arboviruses (Watts and Eldridge 1975, Lee and Rohani 2005, Adams and Boots 2010). Vertical transmission in turn may be supported by sexual transmission from infected males to females and by the survival of long-lived infected females, which can then maintain arboviruses within mosquito populations in the absence of transmission to humans (Rosen 1983, Choochote et al. 2001, Joshi et al. 2003, Kyle and Harris 2008). Because a fitness cost of infection for the mosquito could result in decreased vertical transmission for the virus, determining the fitness cost of infection within both larval and adult 
mosquitoes is therefore essential for understanding the role of mosquitoes as reservoir hosts during periods that are unfavorable for transmission.

Studies to date have shown that within adult female mosquitoes, infection with dengue virus, eastern equine encephalitis virus, and western equine encephalitis virus each resulted in a significant decrease in lifespan (Scott and Lorenz 1998, Mahmood et al. 2004, Maciel-de-Freitas et al. 2011). However, viral-induced reductions in life span did not typically occur until 14 or 21 days after infection. Given that only a small percentage of female mosquitoes will survive that long in nature, the decline in longevity associated with infection is not likely to significantly influence viral transmission, but would likely reduce the potential for long-lived females to support viral persistence in the environment. Alternatively, the fertility of females infected with dengue virus, eastern equine encephalitis virus, and western equine encephalitis virus significantly decreased starting with the first egg batch following infection, which could reduce the rate of vertical transmission.

Relatively little is known about the effects of infection of arboviruses in larvae. Given the distinct physiological and ecological differences between larvae and adults, the effect of infection could be dramatically different between the two stages. To date, there has only been one study that has investigated the effect of dengue infection within larvae. Joshi et al. (2002), found that vertically infected larvae had a longer developmental time and an increased mortality rate, though the data were not analyzed to determine statistical significance.

Understanding the fitness cost of vector-borne pathogens on their invertebrate hosts has implications not only for viral epidemiology but also for virus evolution 
(Lambrechts and Scott 2009). This is because knowing the magnitude and nature of the effect of infection will help to predict the long-term changes in virus-vector interactions resulting from the evolutionary response of the invertebrate hosts to mitigate fitness costs. Understanding the relative fitness costs of infection associated with different viral genotypes could help us predict which genotypes will succeed evolutionarily.

Understanding the fitness effects of infection is particularly relevant for dengue virus, which has emerged over the last 70 years to become the preeminent arboviral threat to human health (Guzman et al. 2010). 2.5 billion people live in areas of endemic dengue transmission and an estimated 100 million people per year are infected with one of the four viral serotypes (Halstead 2007). Dengue epidemics are now occurring on a cyclical basis every 3-4 years in many countries throughout Southeast Asia and South America, and the reservoir hosts in which the virus persists during inter-epidemic periods is unclear (Chen and Vasilakis 2011). In addition, the rapid global spread of invertebrate hosts and novel dengue genotypes has been bringing together new vector-virus combinations whose interactions have varying repercussions for the epidemiology of human infections, and understanding how these interactions may change evolutionarily may aid in management strategies (Lambrechts et al. 2009).

Here we report the results of experiments in which the fitness cost of dengue infection within Ae. aegypti and Ae. albopictus larvae was assessed. We investigated the fitness cost of infection using two dengue-2 genotypes, which differ in their virulence in humans (Leitmeyer et al. 1999). Larvae often develop within nutritionally poor environments, therefore were reared larvae at two different resource levels to investigate the effect of resource level on infection outcome (Muturi et al. 2012). We found that the 
effect of dengue infection was sex-specific; infection in females was relatively cost-free, while infected males had a decreased cephalothorax length and increased development time. Therefore, female mosquitoes infected through vertical transmission will not have increased mortality rates or a decreased life span as a result of infection and will likely to able to transmit dengue to humans. Among infected larvae, there was no significant difference between the two dengue- 2 genotypes. The resource input that larvae were given did not influence the infection rate or development time of infected larvae, but did result in a marginally significant decrease in the cephalothorax length, providing limited support for the hypothesis that resource levels within the larval habitat could influence the effect of dengue infection within mosquito larvae.

\section{MATERIALS and METHODS}

Mosquito Colonies. The Ae. aegypti colony used in this study was established from adults collected in Palm Beach County, Florida, and had been colonized for less than 4 years. The Ae. albopictus colony was established from adults that were field caught in Louisville, Kentucky and had been in the laboratory for less than 1 year. Mosquitoes were maintained in an insectary at $28-30 \mathrm{C}$, relative humidity of $70 \%$, with a $14: 10$ hour light:dark cycle. Larvae were hatched and reared in plastic containers of water and fed tetramin fish food ad libitum. Pupae were transferred to screened cages and emergent adults were maintained on an ad libitum diet of a $10 \%$ sucrose solution. Adult females were fed regularly on hog's blood.

Virus Strains and Cell Culture. Two dengue-2 viral genotypes, representing the Southeast Asian (SEA) (D00-0137) and American (AM) (IQT-2124) genotypes were used in this study. Viral stocks were propagated in the Aedes albopictus derived C6/36 
cell line. $\mathrm{C} 6 / 36$ cells were maintained at $32^{\circ} \mathrm{C}$ with $5 \% \mathrm{CO}_{2}$ in minimal essential medium (MEM), 10\% fetal bovine serum (FBS), $2 \mathrm{mM} \mathrm{L-glutamine,} 100 \mu \mathrm{M}$ non-essential amino acids (NEAA), $100 \mu \mathrm{g} / \mathrm{mL}$ of streptomycin, and $100 \mathrm{units} / \mathrm{mL}$ of penicillin [pen/strep]. In $25-\mathrm{cm}^{2}$ flasks confluent $\mathrm{C} 6 / 36$ monolayers were inoculated at a multiplicity of infection of 0.01 (approximately 1 genome equivalent/ 100 cells) with dengue virus in 3 $\mathrm{mL}$ of maintenance media (MEM, 5\% FBS, $2 \mathrm{mM}$ L-glutamine, $100 \mu \mathrm{M}$ NEAA, and 100 $\mu \mathrm{g} / \mathrm{mL}$ of pen/strep), incubated for 90 minutes at $32^{\circ} \mathrm{C}$, then supplemented with an additional $3 \mathrm{~mL}$ of maintenance media and were incubated at $32^{\circ} \mathrm{C}$ with $5 \% \mathrm{CO}_{2}$ for 5 days. Vero cells were utilized to determine viral infection and were maintained at $37 \mathrm{C}$ with $5 \% \mathrm{CO}_{2}$ in MEM supplemented with $10 \% \mathrm{FBS}, 2 \mathrm{mM}$ L-glutamine, $100 \mu \mathrm{M}$ NEAA, and $100 \mu \mathrm{g} / \mathrm{mL}$ of pen $/ \mathrm{strep}$.

Experimental Infection. Mosquito eggs were hatched in a $50 \mathrm{ml}$ conical tube with $49 \mathrm{ml}$ of distilled water and $1 \mathrm{ml}$ of Luria broth at $28^{\circ} \mathrm{C}$. After 24 hours, $1^{\text {st }}$ instar larvae were collected, rinsed in distilled water and then transferred into MEM. Using a micro-pipettor, larvae were transferred into the $25-\mathrm{cm}^{2}$ flasks that were inoculated with either dengue or MEM (control) and were then placed in a $32 \mathrm{C}$ incubator. Every 24 hours for 3 days, larvae were removed from $25-\mathrm{cm}^{2}$ flasks and counted, rinsed in MEM, and then transferred into a new $25-\mathrm{cm}^{2}$ flask containing dengue virus. After 72 hours of exposure to dengue, larvae were rinsed three times in distilled water and were transferred into 6well plates and reared to pupation in the absence of dengue at a density of 1 larvae per 4 $\mathrm{ml}$ of water at $28^{\circ} \mathrm{C}$. Larvae were reared at two resource levels: larvae received either a $0.1 \mu \mathrm{g}$ (low treatment) or $0.5 \mu \mathrm{g}$ (high treatment) of a 1:1 mixture of brewers yeast and lactalbumin per diem. 6-well plates were checked every 12 hours to maintain constant 
density and resource levels. When pupae emerged, development time was recorded, sex was identified using a dissecting microscope, and cephalothorax length was measured to the nearest $1 / 100^{\text {th }} \mathrm{mm}$ using a digital caliper; larval mortality was also recorded. Pupae were stored at $-80^{\circ} \mathrm{C}$ until assayed to determine infection status.

Assay for Infection Status. Individual pupae were homogenized in $75 \mu$ l of inoculation media (100 ml MEM, $2 \mathrm{ml} \mathrm{NEAA,} 100 \mu \mathrm{g} / \mathrm{mL}$ pen/strep \& $1 \mathrm{ml}$ fungizone) in $1.5 \mathrm{ml}$ micro-centrifuge tubes by manual grinding for 30 seconds with a polycarbonate pestle. Two hundred $\mu l$ of inoculation media was then added to each tube, which was then centrifuged at $3000 \mathrm{rpm}$ for $15 \mathrm{~min}$ at $4^{\circ} \mathrm{C}$. Twenty four well plates of Vero cells were then inoculated with pupal supernatant, incubated at $37^{\circ} \mathrm{C}$ with $5 \% \mathrm{CO}_{2}$ for 90 minutes, overlaid with $0.8 \%$ methylcellulose MEM supplemented with $2 \%$ FBS, $2 \mathrm{mM} \mathrm{L-}$ glutamine, and $100 \mu \mathrm{g} / \mathrm{mL}$ of pen $/ \mathrm{strep}$, and incubated for 5 days at $37^{\circ} \mathrm{C}$ with $5 \% \mathrm{CO}_{2}$. Vero cells were fixed by immersion in methanol for 20 minutes, rinsed in a dry milk buffer [3.5\% dry milk, 1x phosphate-buffered saline (PBS)] and air-dried. Each well was incubated with $200 \mu$ l of buffer containing anti-DENV-2 monoclonal antibodies (MAb 4G2, World Arbovirus Collection) diluted 1:1000 in dry milk buffer) for 90 minutes at $37^{\circ} \mathrm{C}$. Plates were washed twice in buffer followed by incubation of each well using a peroxidase labeled secondary antibody diluted 1:500 in buffer for 60 minutes at $37^{\circ} \mathrm{C}$. Plates were then rinsed in PBS, air dried for 25 minutes and $100 \mu$ l of peroxidase substrate was added to each well. Two hundred and ninety three pupae were analyzed for the presence of dengue virus; individual titer data for positive samples was not determined. Dengue positive samples were re-assayed to confirm viral presence. 
Statistical Analysis. To analyze the effect of dengue infection in larvae, we used a generalized linear mixed model using the Satterthwaite degrees of freedom approximation to accommodate unequal sample sizes. All analyses were done in SAS 9.3 using PROC GLIMMIX.

To improve normality, development time was log-transformed and cephalothorax length was square root transformed prior to statistical analysis. To determine the larval infection rate we assessed the dependence of the binomial response variable infection status on the fixed predicator variables genotype, species, sex and their interactions. Mortality was calculated as the percentage of larvae that survived to the pupal stage. We assessed the dependence of the binomial response variable pupal status on the fixed predictor variables species, genotype, and block. To estimate the affect of dengue infection on development time and cephalothorax size, we assessed the dependence of the continuous variables cephalothorax size and development time on the fixed predictor variables sex, infection status, resource input, species, genotype and their interactions. Each analysis began with a fully factorial model and we performed sequential model reduction to identify best-fit models. Significant main effects and interactions were followed by pairwise contrasts of least-square means using the Bonferroni adjustment for multiple comparisons.

\section{RESULTS}

A total of 112 Ae. albopictus and 181 Ae. aegypti were exposed to either dengue virus or a control treatment to investigate the cost of viral infection. Overall, 35 of the 244 larvae that were exposed to dengue were determined to be dengue positive by ELISA and none of the 49 control larvae were infected. 
We found that the infection rates differed between larvae inoculated with different genotypes and between the two mosquito species; a significantly higher percentage of larvae became infected with the American (AM) genotype relative to the Southeast Asian (SEA) genotype and Ae albopictus were significantly more susceptible to dengue than Ae. aegypti (Table 3, Fig 6 B). The titers of the AM and SEA genotypes to which larvae were exposed were very similar $\sim 1.4 \times 10^{6} \mathrm{ffu} / \mathrm{ml}$ respectively, and therefore differences in infection rate are likely not attributable to exposure method.

Table 3. Mixed model analysis of variance statistics for the best-fit model describing predictors of infection rate. This analysis uses maximum likelihood methods to calculate approximate F-statistics. Denominator degrees of freedom were calculated using the Satterthwaite approximation. Stars indicate level of statistical significance $(*<0.05$, $* *<0.01, * * *<0.001)$.

\begin{tabular}{|ccccc|}
\hline Source & Num DF & Den DF & F Value & Pr $>$ F \\
\hline species & 1 & 239 & 8.59 & $* * *$ \\
sex & 1 & 239 & 0.68 & ns \\
genotype & 1 & 239 & 6.53 & $* *$ \\
genotype*species & 1 & 239 & 0.04 & ns \\
\hline genotype*sex & 1 & 239 & 5.57 & $* *$ \\
\hline
\end{tabular}

We analyzed the pairwise comparisons within the species by genotype interaction to test the a priori hypothesis that the dengue genotypes may infect the mosquito species at a different rate. We found significant species by genotype interactions; the percentage of Ae. albopictus larvae infected with the AM genotype was significantly higher than then percentage of Ae. aegypti infected with the SEA genotype (Fig 6B). The sex by genotype interaction was also significant and pairwise comparisons indicate that was driven by the infection rate of male mosquitoes exposed to the AM genotype (Fig 6A). The three-way genotype by species by sex interaction was not significant and was dropped from our best-fit model. 

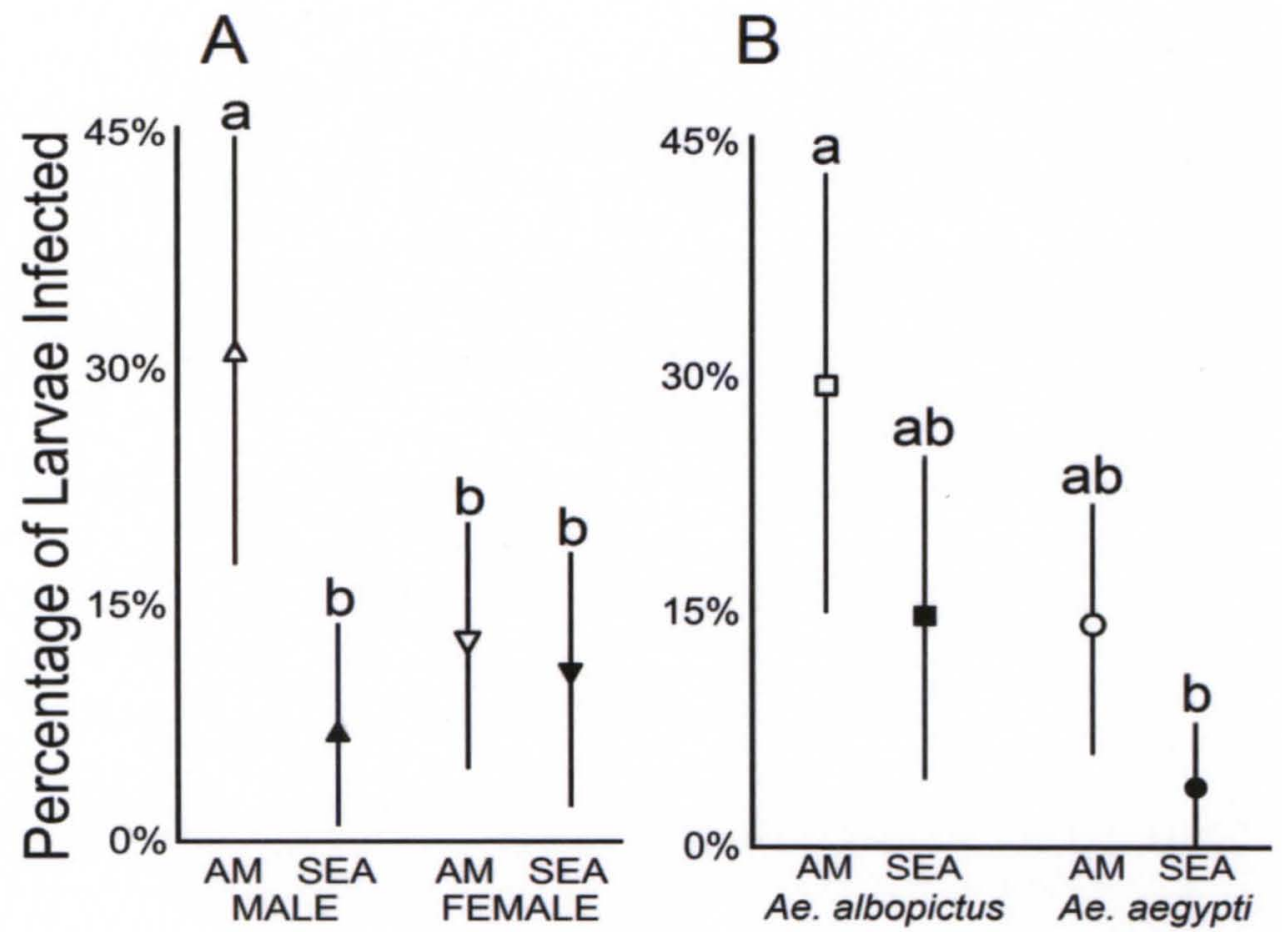

Figure 6. Larval infection rates Mean infection rate with $95 \%$ confidence intervals of (A) sex by genotype interaction and (B) species by genotype interaction. The letters over the estimates indicate the results of pairwise comparisons of each interaction from a logistic regression with (A) genotype by sex or (B) genotype by species as predictor variables, and viral presence as the response variable. Each letter that appears multiple times designates pairs of interactions that did not differ significantly at $\mathrm{p}=0.05$, controlling for an overall alpha across multiple comparisons of 0.05 .

Larvae exposed to dengue did not have an overall increased mortality rate relative to the control group regardless of the dengue-2 genotype to which they were exposed (Table 4, Fig 7). Similarly, there was no consistent difference across genotype in the mortality they caused in Ae. aegypti and Ae. albopictus.

Table 4. Mixed model analysis of variance statistics for the best-fit model describing predictors of mortality rate. Stars indicate level of statistical significance $(*<0.05, * *<0.01$, $* * *<0.001)$.

\begin{tabular}{|ccccc|}
\hline Source & Num DF & Den DF & F Value & Pr $>$ F \\
\hline species & 1 & 335 & 0.91 & $n s$ \\
genotype & 2 & 335 & 0.73 & $n s$ \\
genotype*species & 2 & 335 & 3.28 & $*$ \\
\hline
\end{tabular}


However there was a significant species-dependent effect of dengue genotype, and prior to adjusting for multiple comparisons, there were two marginally significant comparisons: the mortality rate of Ae. albopictus larvae rate of exposed to the AM genotype was higher than that of the Ae. albopictus control $(\mathrm{p}=0.06)$ and the mortality rates differed between control treatments for the two species ( $\mathrm{p}=0.07)$ (Table 4, Fig 7).

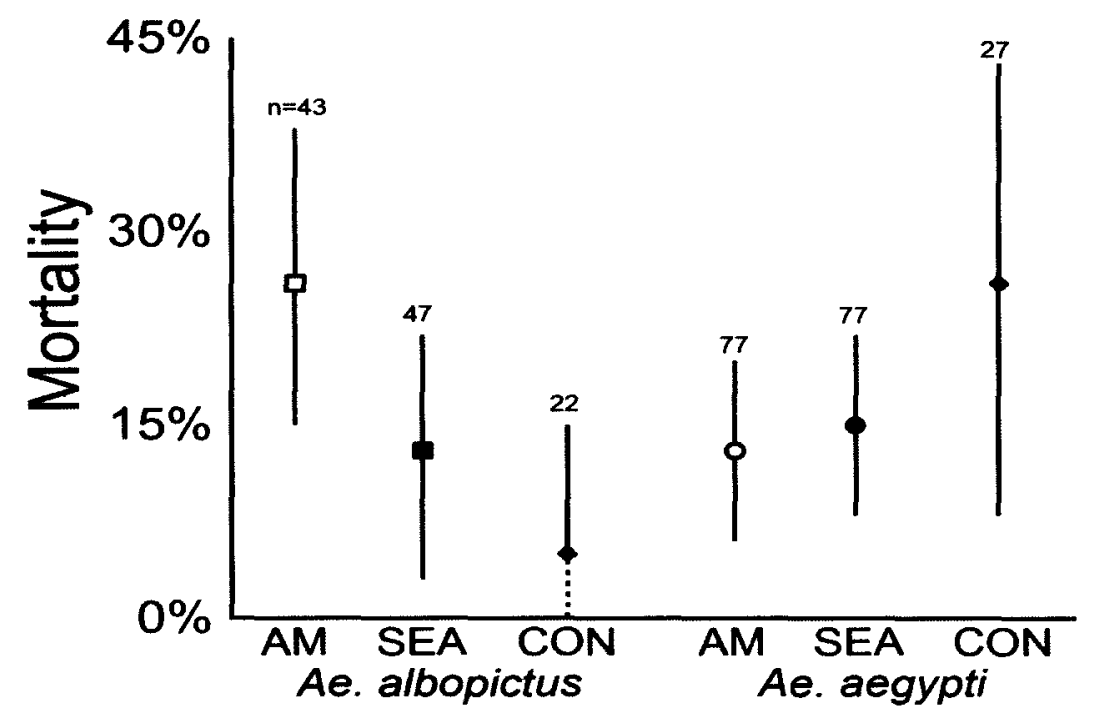

Figure 7. Mean larval mortality rate with $95 \%$ confidence intervals shown by species and exposure treatment. The number of larvae in each treatment is indicated above each estimate.

For both development time and cephalothorax length there was no significant effect of genotype among infected larvae. Therefore we combined the two genotypes in our analysis and looked for the overall effect of dengue infection. The best-fit model describing differences in development time detected a significant 3-way sex by infected by input interaction which was driven by infected male mosquitoes that had longer development times at both the high and low resource inputs (Table 5, Fig 8B). The resource input larvae received significantly influenced development time; larvae receiving the high resource input developed significantly faster than larvae given the low resource input (Fig 8A). Notably, while exposure to the SEA genotype resulted in significantly longer development times than exposure to the AM genotype (Fig 8A), there 
was no significant difference in the development time between SEA and AM infected larvae. Ae. albopictus larvae took significantly longer to develop than Ae. aegypti larvae (data not shown). There was a sex-dependent overall effect of dengue infection on the length of time it took for larvae to pupate; infected males took significantly longer to develop than uninfected males, while there was no significant difference in development time between infected females and uninfected females, $(\mathrm{p}=0.004)$ (Table 5, Fig 8B).

Table 5. Mixed model analysis of variance statistics for the best-fit model describing predictors of development time. Stars indicate level of statistical significance $(*<0.05$, $* *<0.01, * * *<0.001)$.

\begin{tabular}{|ccccc|}
\hline Source & Num DF & Den DF & F Value & Pr $>$ F \\
\hline species & 1 & 230.3 & 18.53 & $* * *$ \\
genotype & 1 & 233 & 4.4 & $*$ \\
infected & 1 & 233.5 & 4.4 & $*$ \\
resource input & 1 & 232.2 & 34.62 & $* * *$ \\
sex*infected & 2 & 233.4 & 24.72 & $* * *$ \\
sex* resource input $^{*}$ infected & 3 & 233.2 & 2.74 & $*$ \\
\hline
\end{tabular}

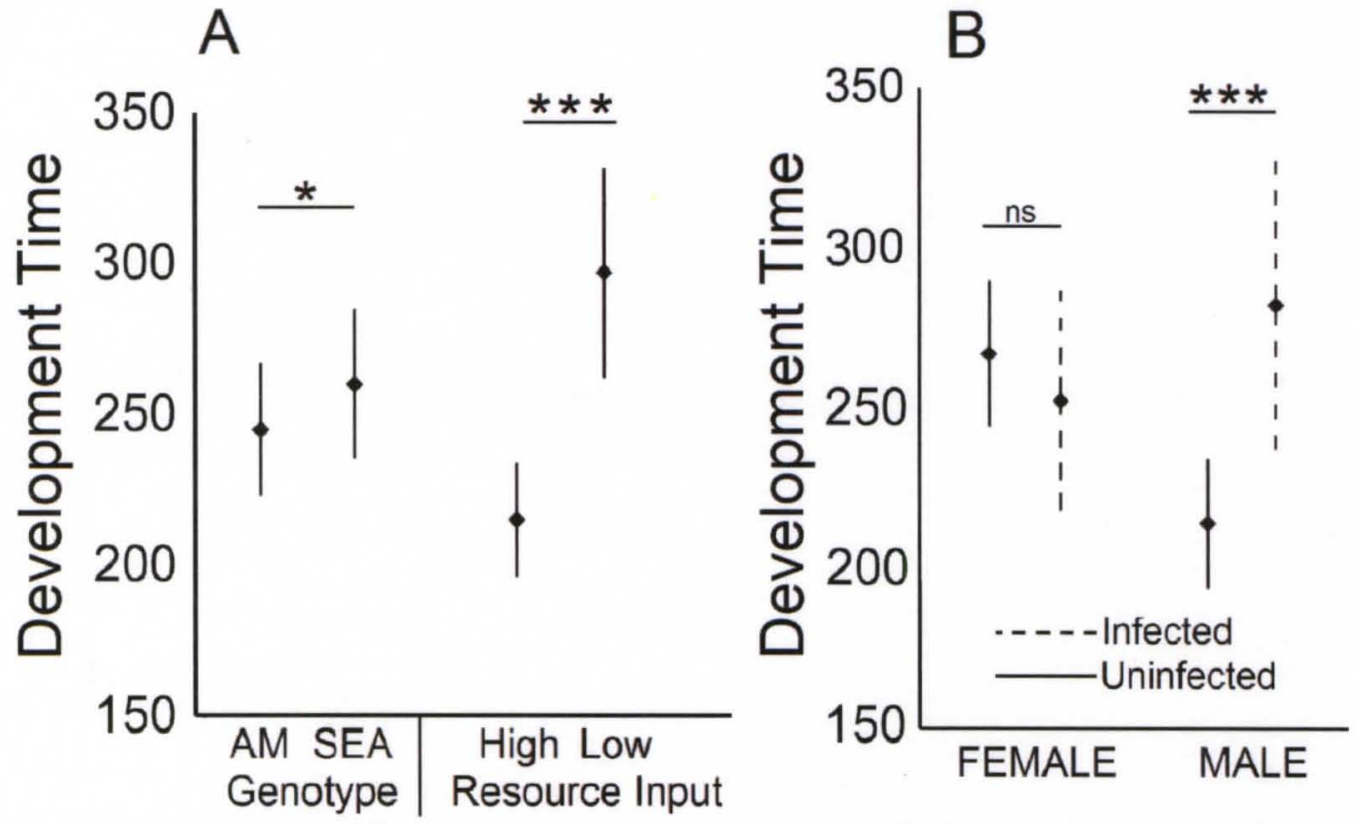

Figure 8. Development Time least-square means with $95 \%$ confidence intervals for (A) genotype and resource input or (B) sex by infection status interaction. The stars over the estimates indicate the significance level $(*<0.05, * *<0.01, * * *<0.001)$ and results of pairwise comparisons of (A) main effects or (B) sex by infection status interaction from a general linear model with (A) genotype and resource input or (B) sex by infection status interaction as predictor variables, with development time as the response variable. 
The two resource inputs that the larvae were given led to the emergence of two distinct pupal sizes: larvae given the lower resource input were significantly smaller than larvae given the high resource input, and as expected in these sexually dimorphic species, females overall were larger than males (Fig 9). Ae. albopictus were significantly larger than Ae. aegypti (Table 6). Overall the cephalothorax length infected larvae was not significantly different from uninfected larvae (Table 9, Fig 9A). However, there was a significant sex by infection status interaction; infected males had a significantly smaller cephalothorax length compared to uninfected males, while there was no significant difference in cephalothorax size between infected females and uninfected females (Fig 9B). The resource input by infected status interaction was a significant, which provides some limited support for the hypothesis that resource levels can affect mosquito response to infection. Pairwise contrasts exploring this overall difference showed that at high resource inputs there was no effect of infection ( $\mathrm{p}<0.17$, but at low resource inputs infection caused a marginally significant decreased cephalothorax size $(\mathrm{p}=0.08$ before correction for multiple comparisons).

Table 6. Mixed model analysis of variance statistics for the best-fit model describing predictors of cephalothorax length. Stars indicate level of statistical significance $(*<0.05$, $* *<0.01, * * *<0.001)$.

\begin{tabular}{|ccccc|}
\hline Source & Num DF & Den DF & F Value & Pr $>$ F \\
\hline species & 1 & 234 & 21.78 & $* \star *$ \\
resource input & 1 & 234 & 167.57 & $* \star *$ \\
sex & 1 & 234 & 243.05 & $* \star *$ \\
\hline infected & 1 & 234 & 0.56 & ns \\
\hline resource input*infected & 1 & 234 & 3.71 & $*$ \\
\hline sex*infected & 1 & 234 & 4.97 & $*$ \\
\hline
\end{tabular}




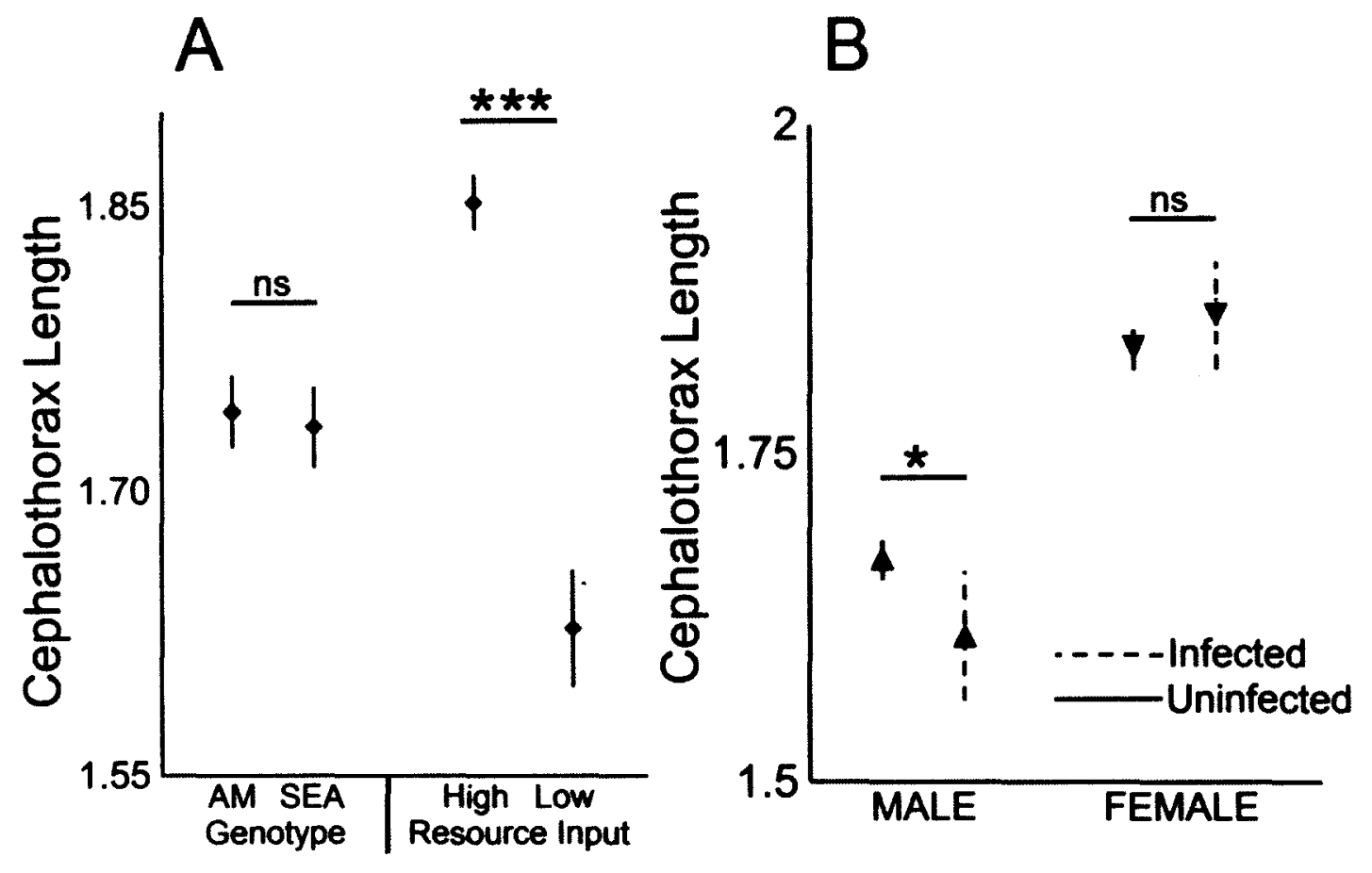

Figure 9. Cephalothorax length least-square means with $95 \%$ confidence intervals for (A) genotype and resource input or (B) sex by infection status interaction. The stars over the estimates indicate the significance level $(*<0.05, * *<0.01, * * *<0.001)$ and results of pairwise comparisons of $(A)$ main effects or $(B)$ sex by infection status interaction from a general linear model with (A) genotype and resource input or (B) sex by infection status interaction as predictor variables, with development time as the response variable.

\section{DISCUSSION}

Our results have two interesting implications regarding the role of vertical transmission in dengue epidemiology. The absence of a fitness cost associated with dengue infection in females, either with respect to development time or cephalothorax size (Tables 5 \& 6, Figs 8B \& 9B) indicates that the infected females are likely to emerge as adults and transmit dengue both horizontally to humans and vertically to their progeny. In contrast, we found that infected males had a significantly longer development time and a significantly smaller cephalothorax relative to uninfected males. Such dengue-induced changes to male life history traits could influence mosquito population dynamics and 
reduce the rate of sexual transmission within mosquito populations.

One potential cause of the sex-dependent fitness costs of dengue infection could be the differential selective pressures that males and females experience; distinct life history trajectories between females and males may influence investment strategies between offspring and competing for mating opportunities, which could select for a lower investment in immune defense among males (Schimd-Hempel 2005). When challenged with a bacterial infection, female crickets and scorpion flies mounted a more robust immune response to infection relative to males (Zuk and Stoehr 2002).

Understanding the relative fitness cost of infection with different dengue genotypes is important for predicting the effects of vector-driven selection in dengue evolution. In this study we used two dengue- 2 genotypes to determine if there is a differential fitness cost of dengue infection with either the SEA or AM genotype. The SEA genotype has a number of consensus mutations in the 5' $^{\prime}$ and $3^{\prime}$ NCR of the dengue genome that cause higher viral replication within both humans and mosquitoes (Leitmeyer et al. 1999, Cologna and Rico-Hesse 2003, Anderson and Rico-Hesse 2006), We hypothesized that a similar difference in replication could occur in mosquitoes, causing the SEA genotype to have a higher fitness cost than the AM genotype within larvae. However, we were did not detect a significant difference in fitness effects between SEA or AM infected larvae as measured by either development time, cephalothorax length, or mortality. This suggests that SEA infection within mosquitoes may not be associated with a higher fitness cost relative the AM genotype. This lack of difference in fitness costs has significant implications for the evolution of dengue virus: without a concomitant fitness cost due to consequences of virulence in the mosquitoes 
that vector it, the SEA genotype, with its increased virulence in humans, could be expected to continue to spread.

One caveat to this prediction arises from the observation that although infection with SEA and AM did not result in different development times, exposure to SEA resulted in significantly longer development time than did exposure to the AM genotype. While this result is puzzling, it is not without precedent; Maciel-de-Freitas (2011) found that dengue exposed but uninfected adults had a higher mortality rate compared to infected adults. One possible cause of this phenomenon could be that the immune response of some larvae was able to clear the infection and the cost of immune activation resulted in an increased developmental time. If this were indeed the case then there is an increased cost of infection associated with SEA infection, which could influence its transmission.

The two primary invertebrate hosts of dengue, Ae. aegypti and Ae. albopictus often develop in nutrient limited environments (Arrivillaga and Barrera 2004). Larval development within nutritionally limiting habitats has been demonstrated to result in reduced immune function (Suwanchaichinda and Paskewitz 1998, Arrivillaga and Barrera 2004, Telang et al. 2011). To test the hypothesis that resource levels within the larval habitat could influence the outcome of dengue infection, we reared a subset of larvae under a reduced resource input to investigate whether resource level could influence the outcome of dengue infection within larvae. We found that the resource input by infected interaction was significant for cephalothorax length; infected larvae given the low resource input were smaller than uninfected larvae given the low input (marginal 
significance, $(\mathrm{p}=0.08)$ prior adjusting for multiple comparisons $)$, which provides limited support for the hypothesis that resource levels can alter the outcome of infection.

This is the first experiment to analyze the infection costs of arbovirus infection in both male and female mosquitoes. While understanding the fitness cost of infection in females has clear implications for human health since female mosquitoes are responsible for the horizontal transmission of pathogens to humans, the results of this study underscore the importance of understanding the effect of infection in male mosquitoes. 


\section{CHAPTER IV}

\section{UTILIZATION OF LARVAL DETRITUS WITHIN THE LARVAL HABITAT}

\section{SUMMARY}

The utilization of detritus sources within the larval habitat by Aedes mosquitoes has important consequences for the outcome of interspecific interactions and mosquito population growth. Several anecdotal observations have indicated that invertebrate detritus may be directly consumed by Aedes larvae, but it has never been thoroughly investigated. In this experiment we tested the hypothesis that invertebrate detritus can be directly consumed by Aedes larvae using the carcasses of mosquito larvae and pupae, which are two types of invertebrate detritus that commonly found within the larval habitat. Overall we found that Ae. aegypti and Ae. albopictus $3^{\text {rd }}$ instar larvae rapidly consumed larval detritus while pupal detritus was consumed at a significantly lower rate. These results indicate that larval Aedes mosquitoes may directly consume certain forms of invertebrate detritus. Because larval detritus was directly consumed, we conducted a second experiment that investigated the survivorship and development of Ae. aegypti larvae when larval detritus was the sole resource input. We found that the presence of larval detritus significantly influenced survivorship and but did not greatly influence larval development. These two experiments indicate that different forms of invertebrate detritus are utilized differently within the larval habitat, and that the direct 
consumption of larval detritus can support the production of adults in larval habitats that lack allochthonous inputs or where such inputs are insufficient.

\section{INTRODUCTION}

Understanding the ecology of mosquito vectors is a crucial challenge to our understanding of vector-borne disease dynamics (Yee et al. 2006). Within artificial containers where many epidemiologically important mosquito species such as Ae. aegypti and Ae. albopictus develop, allochthonous inputs in the form of plant and animal detritus are the primary energy source (Yee and Juliano 2006, Kesavaraju et al. 2007). The ratio and types of detritus sources within the larval habitat significantly influence mosquito population growth, interspecific competition, and the capacity of mosquito populations to vector pathogens (Alto et al. 2005, Murrell and Juliano 2008, Alto 2011). Therefore, understanding how different detritus types influence larval development, and interspecific interactions has many important implications for human health and arbovirus epidemiology.

While larval Ae. aegypti and Ae. albopictus primarily obtain resources within the larval habitat by ingesting fine particulate detritus and microbes, there is anecdotal evidence that they may directly consume animal detritus (Daughtery et al. 2000, Yee et al. 2006, Yee and Juliano 2007). Since the direct consumption of invertebrate detritus could influence the outcome of competitive interactions within the larval habitat, we conducted two experiments to determine if invertebrate detritus was directly consumed, and if direct consumption occurs, how it affected larvae growth and development.

This experiment assessed the direct consumption of larval and pupal detritus by Ae. aegypti and Ae. albopictus larvae and determined the effect of larval detritus on Ae. 
aegypti survivorship, and development. We found that larval detritus was rapidly consumed by both species, while the consumption of pupal detritus occurred at a significantly slower rate. In the second experiment Ae. aegypti larvae were reared under three levels of larval detritus to determine the impact of the direct consumption of larval detritus on survivorship, development time, and pupal cephalothorax length, which is an estimate of adult fitness. The direct consumption of larval detritus significantly increased the survivorship rate of $A e$. aegypti larvae. The level of larval detritus significantly influenced the development time of male mosquitoes but not females; neither male nor

female cephalothorax length was influenced by resource inputs. Overall we report for the first time the direct consumption of invertebrate detritus and the outcome of larval development when larval detritus is the sole detritus input.

\section{MATERIALS and METHODS}

Mosquito Colonies. The Ae. aegypti colony used in this study was established from adults collected in Palm Beach County, Florida, and had been colonized for less than 4 years. The Ae. albopictus colony was established from adults that were field caught in Louisville, Kentucky and had been in the laboratory for less than 1 year. Mosquitoes were maintained in an insectary at $28-30 \mathrm{C}$, relative humidity of $70 \%$, with a $14: 10$ light:dark photoperiod. Larvae were reared in plastic containers $(6 \times 20 \times 20 \mathrm{~cm})$ with $400 \mathrm{ml}$ of reverse osmosis water and were fed tetramin fish food ad libitum. Pupae were transferred to screened cages and emergent adults were maintained on an ad libitum diet of a $10 \%$ sucrose solution. Adult females were fed regularly on hog's blood. 


\section{Experiment 1. Investigation of detritus consumption}

Shumard red oak Quercus shumardii leaves were collected from the University of Louisville campus during the fall of 2009 and served as a basal food resource for mosquito larvae. Leaves were dried at $60^{\circ} \mathrm{C}$ for 48 hours, and stored until use in experiments. Experimental microcosms consisted of cylindrical plastic containers filled with $150 \mathrm{ml}$ of reverse osmosis water, $125 \mathrm{mg}+/-1 \mathrm{mg}$ of senescent dried oak leaves, and $100 \mathrm{ul}$ of microbial inoculum obtained from red oak tree holes on the University of Louisville campus. Leaves were added to the experimental microcosms 3 days prior to the addition of larvae to allow microbial growth to occur. 7 days prior to the start of the experiment, Ae. aegypti and Ae. albopictus eggs were hatched in $49 \mathrm{ml}$ of reverse osmosis water with $1 \mathrm{ml}$ of Luria broth. Twenty-four hours later, $1^{\text {st }}$ instar larvae were collected, rinsed, and reared in containers with $500 \mathrm{ml}$ reverse osmosis water and $250 \mathrm{mg}$ oak leaf detritus. To begin the experiment, $103^{\text {rd }}$ instar larvae were placed into each microcosm, and every microcosm received either $43^{\text {rd }}$ instar larval carcasses or 4 pupal carcasses that had been non-destructively killed through freezing at $-80^{\circ} \mathrm{C}$. The larval or pupal detritus input into each microcosm had a mass of $6 \mathrm{mg}+/-0.3 \mathrm{mg}$. Microcosms were placed in an environmental chamber at $28-30^{\circ} \mathrm{C}$ with a $14: 10$ light:dark photoperiod. Every 24 hours the extent of scavenging was recorded along with the number of larvae in each container. Scavenging was scored on an ordinal scale; the extent of scavenging was scored for each larval or pupal carcass as either completely consumed (1), partially consumed (0.5), or no visible scavenging (0), and the sum of all four scores was recorded for each microcosm on a daily basis. The larval density was maintained at 10 larvae per microcosm throughout the 14-day experiment; pupae that emerged were 
removed and were replaced with 3 rd instar larvae. The water volume within each microcosm was checked often and was maintained at $150 \mathrm{ml}$. The microcosms were monitored every day for 14 days. For each species there were 10 replicates of the larval detritus treatment and 5 replicates of the pupal detritus treatment.

\section{Experiment 2. Effect of larval detritus on larval development}

Experimental microcosms consisted of 6-well plates, each well contained $10 \mathrm{ml}$ of oak leaf extraction water which was prepared 3 days prior to the beginning of the experiment, by placing $500 \mathrm{~g}$ of senescent dried oak leaves in 1 liter of reverse osmosis water and then incubating at $28^{\circ} \mathrm{C}$ for 72 hours. Ae. aegypti eggs were placed into a 50 ml conical tube containing reverse osmosis water and were placed under vacuum pressure to stimulate hatching. 12 hours later, $1^{\text {st }}$ instar larvae were collected and 10 larvae were placed into each well of a 6-well plate containing leaf leachate. Every three days each well was given one of three larval detritus inputs (control-0 carcasses, medium-5 carcasses, or large-10 carcasses). Each treatment had ten replicates. Larval detritus inputs consisted of $3^{\text {rd }}$ instar larvae that were non-destructively killed through freezing at $-80^{\circ} \mathrm{C}$ and were subsequently thawed before adding to microcosms. 6-well plates were placed in an environmental chamber at $28-30^{\circ} \mathrm{C}$ with a $14: 10$ light:dark photoperiod and were checked daily for pupal emergence and mortality for the duration of the experiment. When pupae emerged they were removed from the 6-well plate, development time was recorded, and sex was identified using a dissecting microscope. Pupae were placed into $1.5 \mathrm{~mL}$ micro-centrifuge tubes and stored at $-80^{\circ} \mathrm{C}$ for later analysis. Pupae were subsequently thawed and cephalothorax length was measured to the nearest $1 / 100^{\text {th }} \mathrm{mm}$ using a digital caliper. 


\section{Statistical Analysis}

Experiment 1. To analyze the consumption of larval and pupal detritus, we used an ordinal logistic regression using PROC GLIMMIX (SAS 9.3). We assessed the dependence of the ordinal response variable scavenging score on the fixed predictor variables species, detritus type, and their interaction. Significant main effects and interactions were followed by pairwise contrasts of least-square means using the Bonferroni adjustment for multiple comparisons.

Experiment 2. To analyze the effect of larval detritus on Ae. aegypti growth, development, and survivorship, a two way ANOVA using PROC GLIMMIX to accommodate unequal sample sizes (SAS 9.3) was used. We assessed the dependence of the continuous response variables development time and cephalothorax length on the fixed predictor variables resource input, sex and their interaction. We assessed the dependence of the binomial response variable survivorship on the fixed predictor variable resource input using a Chi Squared test performed in PROC FREQ (SAS 9.3).

\section{RESULTS}

Experiment 1-The patterns of larval and detritus consumption were indistinguishable for Ae. aegypti and Ae. albopictus ( $\mathrm{p}=1$ main effect of species; $\mathrm{p}=1$ species by detritus type interaction). Larval detritus was directly consumed rapidly by both species while the consumption of pupal detritus occurred at a much lower rate $(\mathrm{P}<0.0001$, Fig. 10). After 48 hours the majority of larval detritus was consumed, but it then took several days before the remaining small pieces of larval detritus were consumed. There was no larval detritus remaining in any microcosm after 8 days. Conversely, the consumption of pupal detritus only became apparent after approximately 7 days had elapsed and most pupal 
carcasses remained intact and unconsumed throughout the duration of the 14-day experiment. The results indicate quite clearly that larval and pupal detritus are consumed differently within the larval habitat.

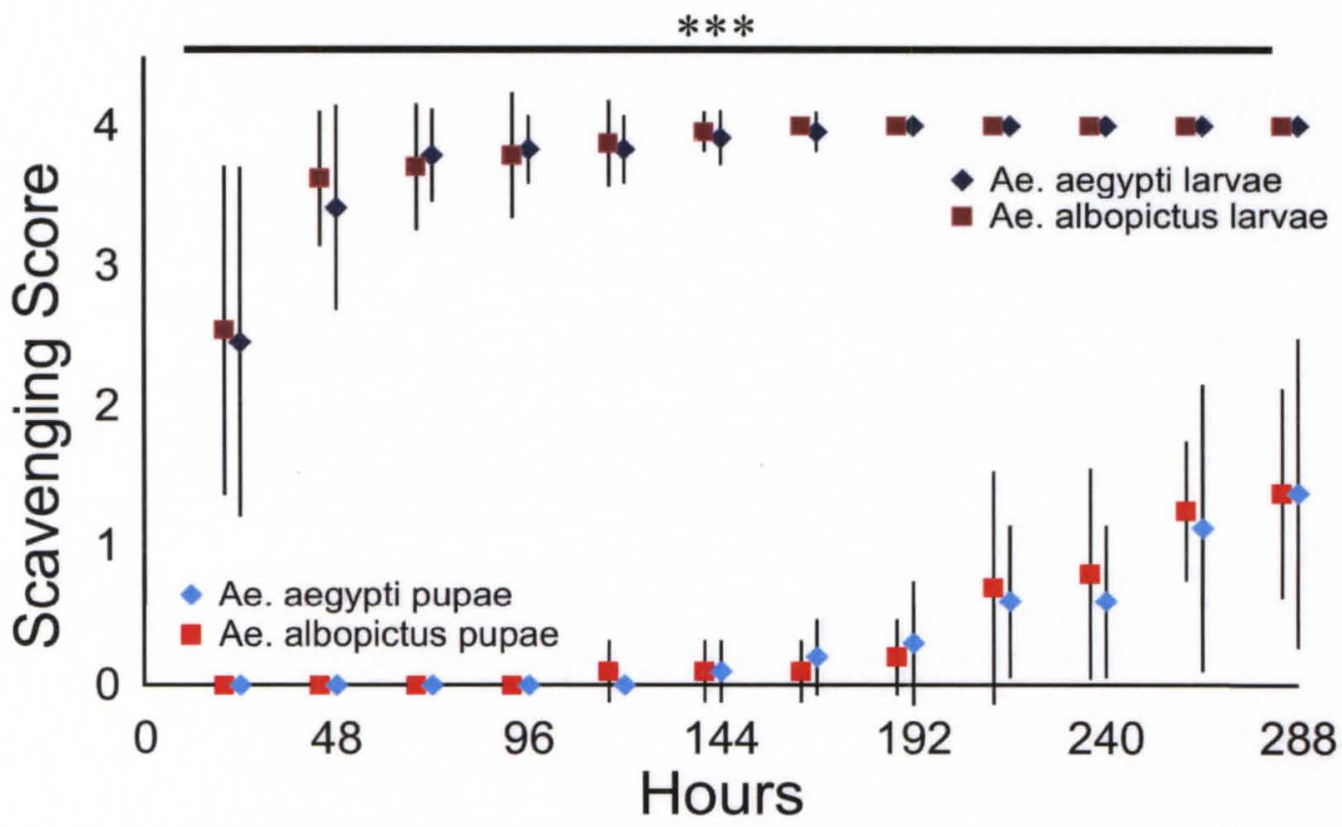

Figure 10. Consumption of larval and pupal detritus by Ae. aegypti and Ae. albopictus larvae. Mean scavenging score (on a scale of 0-4) with standard deviation for each speciesdetritus combination. At each time point the scavenging score between larval and pupal detritus was significantly different (ordinal logistic regression, SAS PROC GLIMMIX, all $\mathrm{p}<0.05)$.

Experiment 2- 300 Ae. aegypti larvae were reared within 6-well plates under three input levels of larval detritus to determine the effect of larval detritus on larval growth and development. The addition of larval detritus significantly influenced the survivorship (Fig 11A). Without any larval detritus input, no larvae successfully developed, indicating that the experimental leaf leachate alone cannot support the development of larva. Larvae receiving the 10 -carcass input had a significantly higher survival rate compared to the 5carcass input, and both had a significantly higher rate than the control treatment $(\mathrm{p}<0.001$ Fig 11A). Increased larval detritus inputs led to a decrease in the development time of male but not female Ae. aegypti larvae ( $\mathrm{p}=0.05$, sex by input level; Fig 11B). While there 
was a trend of increasing cephalothorax length in containers receiving the 10 -carcass input, cephalothorax length was not significantly larger in male or female larvae receiving the 10-carcass input (Fig 11C).
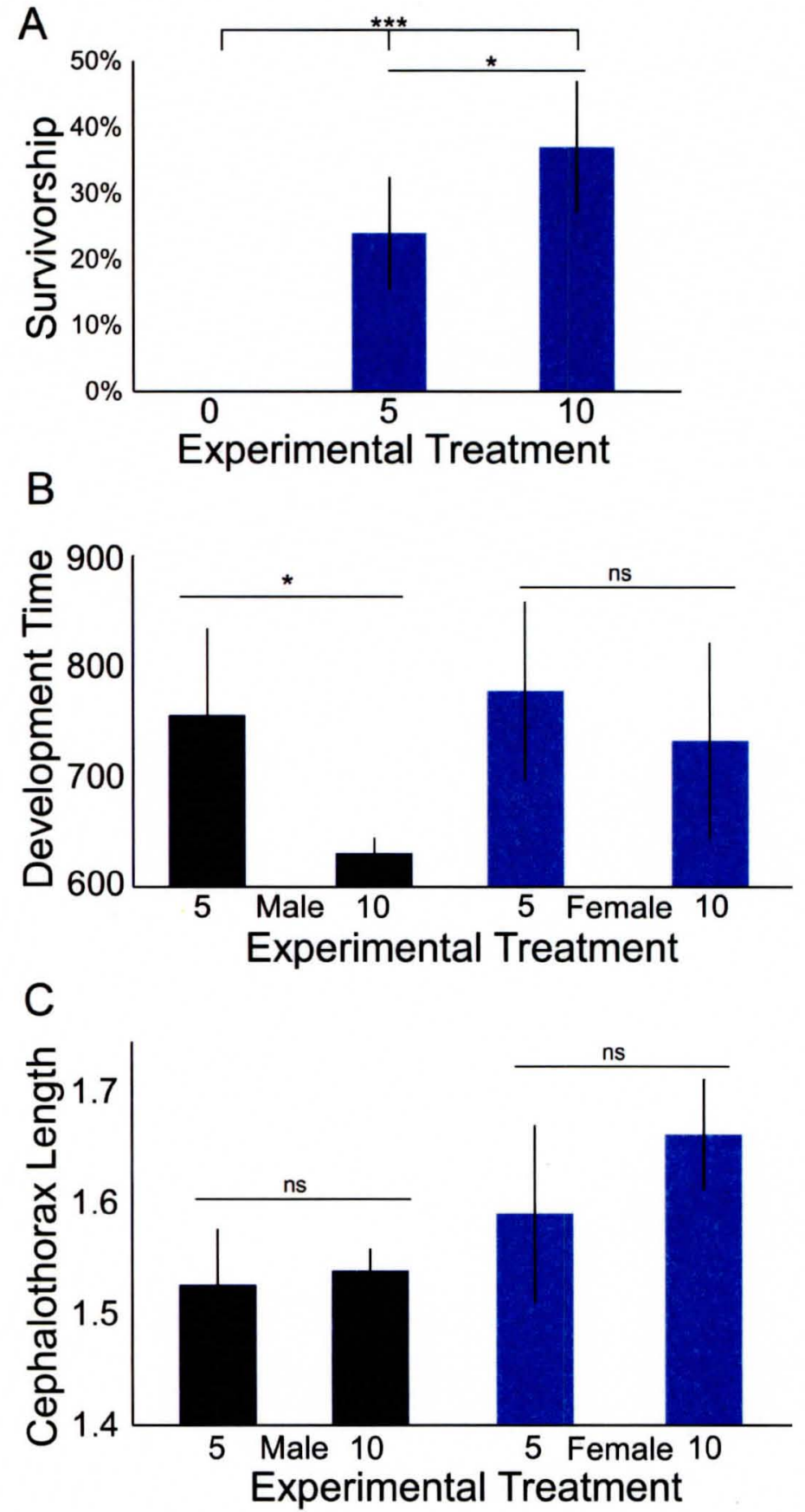

Figure 11. Means $+/$ - standard deviation for (A) survivorship, (B) development time, and (C) cephalothorax length of Ae. aegypti larvae reared with three larval detritus inputs. The stars over the estimates indicate the significance level from Chi Squared test, SAS PROC FREQ (A) or two way ANOVA, SAS PROC GLIMMIX (B, C) $(*<0.05, * *<0.01, * * *<0.001)$. 


\section{DISCUSSION}

The consumption patterns of Ae. aegypti and Ae. albopictus larvae were remarkably similar; larvae of both species were able to recognize the presence of larval detritus within the habitat and foraged upon it at a parallel rate (Fig. 10). Nucleotides act as a strong phagostimulant for mosquito larvae and given their rapid consumption it is possible that the dead carcasses released nucleotides, which attracted larvae to forage around them (Clements 1992). While pupal detritus was directly consumed to a lesser extent, the delayed consumption suggests that a certain amount of microbial decomposition was necessary before larvae could consume the remaining carcass. This experiment demonstrates for the first time that larval detritus is consumed rapidly within the larval habitat. In addition to potentially influencing the role of larval detritus on mosquito development, the rapid consumption of larval carcasses has epidemiological consequences since it could be a mechanism for the horizontal transmission of pathogens within the larval habitat (Miller et al. 1978).

The reduced consumption rate of pupal carcasses could be attributed to the thickness of the pupal cuticle. When fully formed, mosquito pupae have a sclerotized cuticle that is 3 um thick, which is much thicker than larval cuticle (Clements 1992). The mouthparts of predacious mosquito larvae give some indication of the mechanism of larval scavenging. Larvae of the Sabethine genus, of which a number are facultative predators, have enlarged mandibles and maxillae indicating that these mouthparts may be useful for the consumption of invertebrate detritus (Clements 1992). While Ae. aegypti and Ae. albopictus do have strong mandibles (Clements 1992), it possible that they are unable to chew through the sclerotized cuticle of mosquito pupae and will likely be 
unable to directly consume types of invertebrate detritus that possesses a thick cuticle until their cuticles are broken down by microbes (Yee and Juliano 2007).

The decay rate of different forms of plant detritus has been shown to influence mosquito development in different ways and it is possible that this may also apply to animal detritus (Murrell and Juliano 2009, Costanzo et al. 2011). The ephemeral nature of larval detritus in the larval habitat may be selectively beneficial to the larvae that directly consume it, whereas the prolonged decomposition of invertebrate carcasses may stimulate microbial population growth yielding a smaller benefit to a greater number of larvae within the habitat (Yee et al. 2007).

While allochthonous inputs may be the primary energy source in larval habitats, (Yee and Juliano 2006) our study suggests that autochthonous detritus in the form of dead mosquito larvae can have a significant effect on larval development and survivorship (Fig 11). The direct consumption of larval detritus supported larval development and resulted in the production of pupae (Fig 11A). This indicates that within larval habitats that lack sufficient resources to support development, the direct consumption of larval carcasses could support the development of surviving larvae.

Among mosquitoes, there is a distinct sexual dimorphism between male and female mosquitoes. Male mosquitoes develop faster and have a smaller body size compared to female mosquitoes. Our data indicates that the responses of male and female larvae to detritus types may also be distinct. We found that male larvae developed significantly faster when given the high detritus input whereas there was no significant difference in female development time. Future research is necessary to better understand the ways in which male and female larval development is influenced by resource type. 


\section{CHAPTER V}

\section{INTRASPECIFIC AND INTERSPECIFIC LARVAL COMPETITION BETWEEN THE DENGUE VECTORS AE. AEGYPTI AND AE. ALBOPICTUS}

\section{SUMMARY}

Understanding the effect of interspecific and intraspecific interactions between mosquito larvae is important for understanding vector-borne diseases like dengue virus, because the effects of resource competition between larvae carry over into the adult stage, significantly influencing adult life history traits, mosquito susceptibility to arboviruses, and the geographic distribution of mosquito species.

The outcome of interspecific competition between larvae is often contextdependent; the quantity and quality of detritus within the larval habitat determines both the intensity of resource competition and competitive outcomes. Larval mortality is often high within artificial containers, and larval carcasses maybe one of the most common detritus sources within larval habitats. Surprisingly, despite its common presence within larval habitats, the impact of larval detritus on interspecific and intraspecific interactions between Ae. aegypti and Ae. albopictus larvae have never been studied. In this experiment we used an addition series experimental design to investigate the outcome of interspecific competition between Ae. aegypti and Ae. albopictus larvae and a substitution series experimental design to investigate the effect of intraspecific competition intensity on larval development when larval detritus was the primary resource. 
We found that Ae. albopictus was the superior competitor relative to Ae. aegypti; cephalothorax length was significantly smaller in Ae. aegypti that developed in the presence of Ae. albopictus. Conversely, Ae. albopictus that developed in conditions of interspecific competition had a significantly larger cephalothorax length, and a significantly shorter development time relative to Ae. albopictus that developed under intraspecific competition. While Ae albopictus survivorship was nearly identical between larvae reared under interspecific and intraspecific competition, Ae. aegypti survivorship was significantly higher under interspecific competition relative to intraspecific competition. These results indicate that development in the presence of Ae.albopictus, may significantly alter Ae. aegypti populations' adult life history traits and susceptibility to arboviruses.

Our data show that intraspecific competition led to significant decreases in survivorship for both Ae. aegypti and Ae. albopictus, while its effect on cephalothorax length and development time were species and sex-dependent. At high larval density, increasing levels of larval detritus significantly affected the development time and cephalothorax length of Ae. albopictus but not Ae. aegypti. We also found that male Ae. aegypti had an inverse response to detritus inputs in terms of development time and cephalothorax length, which suggests that the effect of allochthonous inputs on larval development are not uniform and may be both density and sex-dependent.

\section{INTRODUCTION}

The larval environment has an indirect yet significant impact on the transmission of mosquito-borne pathogens such as dengue virus, malaria, and Yellow fever virus 
(Hume et al. 2011, Alto et al. 2008). As holometabolous insects, mosquitoes have a complex life cycle within an aquatic immature stage from which the adult stage subsequently disperses into the terrestrial environment (Muturi et al. 2012). During the larval stage, a complex set of biotic and abiotic factors interact to shape larval development (Juliano 2009 Kaufman et al. 2010). The amount of resources that larvae are able to accumulate during development determines the life history traits of the adult mosquito (Fig 2) (Clements 1992). Arbovirus transmission and epidemiology is very sensitive to small changes in adult life history traits such as life span and therefore understanding how the environment influences larval development is critical to understanding the dynamics of vector borne disease and developing effective vector control strategies (Dye 1986, Yee et al. 2006 ).

The biotic interactions within artificial containers where many epidemiologically important mosquito species such as Ae. aegypti and Ae. albopictus develop are primarily dominated by resource competition (Arrivillaga and Barrera 2004, Alto et al. 2005). Artificial containers are small transitory pools of water where primary production is low, and survival depends on allochthonous inputs primarily in the form of plant detritus and invertebrate carcasses (Fish and Carpenter 1982). Detritus inputs are often limiting, and inter- and intraspecific competition within artificial containers is common (Alto 2011). This competition influences vector-borne transmission directly and indirectly in three primary ways: by influencing mosquito susceptibility, by influencing mosquito population dynamics, and by influencing mosquito distribution and geographic range (Rey et al. 2006, Alto et al. 2008, Armistad et al.2008).

Multiple mosquito species often inhabit the same container and interspecific 
competitive outcomes are usually context-dependent and asymmetric (Yee 2008, Juliano 2009). When interspecific competition is highly asymmetrical, the competitive exclusion of species across their shared host range may occur, which can significantly influence the geographic distribution of the mosquito species (Juliano 1998, Juliano and Lounibos 2005). While intraspecific competition does not affect the geographic range, interspecific competition significantly influences adult susceptibility to arboviruses and life history traits such as life span and fecundity (Muturi et al. 2011). The two primary vectors of dengue virus, Ae. aegypti and Ae. albopictus co-occur throughout Southeast Asia and the Western Hemisphere and the outcome of their competitive interactions has important implications for dengue epidemiology.

Studies of competition between Aedes aegypti and Aedes albopictus have addressed the role of allochthonous arthropod inputs (Daughtery et al. 2000, Murrell and Juliano 2008). However the inputs used in these studies have not been ones that are likely to be prevalent within artificial containers (Drosophila, crickets). We have shown that invertebrate detritus may differ in the extent to which it is directly consumed by $A e$. aegypti and Ae. albopictus larvae (Chapter 4); such differences may influence the effects of input additions on competitive outcomes. Because in artificial containers mosquito larval density and mortality can both be high, dead larval inputs may be the most common and important detritus source that could affect competition.

In this study we assessed the effect of interspecific competition and intraspecific competition intensity on survivorship, development time, and cephalothorax length of $A e$. aegypti and Ae. albopictus when larval detritus was the primary detritus input. We found that interspecific competition significantly influenced the development and survivorship 
of larvae; Ae. albopictus was the superior competitor relative to Ae. aegypti within mixed species microcosms. Consistent with previous studies, we found that increased larval density had a negative effect on larval development and survivorship (Juliano 1998, Alto et al. 2008, Reiskind and Lounibos 2009). The extent to which density influenced development time and cephalothorax length was influenced by both species and sex; at high density increasing inputs of larval detritus did not affect either development time or cephalothorax length for Ae. aegypti. We also found that male Ae. aegypti were inversely effected by detritus inputs relative to other species-sex combinations suggesting that detritus inputs may affect the development of males and females in different ways.

\section{MATERIALS and METHODS}

Mosquito Colonies. The Ae. aegypti colony used in this study was established from adults collected in Palm Beach County, Florida, and had been colonized for less than 4 years. The Ae. albopictus colony was established from adults that were field caught in Louisville, Kentucky and had been in the laboratory for less than 1 year. Mosquitoes were maintained in an insectary at $28-30^{\circ} \mathrm{C}$, relative humidity of $70 \%$, with a $14: 10$ light:dark photoperiod. Larvae were hatched and reared in plastic containers of water and fed tetramin fish food ad libitum. Pupae were transferred to screened cages and emergent adults were maintained on an ad libitum diet of $10 \%$ sucrose solution. Adult females were fed regularly on hog's blood.

Experimental Design. Experimental microcosms consisted of cylindrical plastic containers filled with $200 \mathrm{~mL}$ of reverse osmosis water, $125 \mathrm{mg}+/-1 \mathrm{mg}$ of senescent dried red oak leaves Quercus shumardii and $1 \mathrm{~mL}$ of water obtained from red oak tree holes on the University of Louisville campus the tree hole water served as a inoculum for 
microorganisms. Microcosms were established three days prior to beginning the experiment, to allow for microbial growth. Mosquito eggs were synchronously hatched 1 day before the experiment began, and 24 hours later $1^{\text {st }}$ instar larvae were collected, rinsed and added to each container to start each competition. We used a substitution series of Ae. aegypti: Ae. albopictus of $(20: 0,10: 10,0: 20)$ to investigate the effect of interspecific competition between species, and an addition series of $(10: 0,20: 0)$ to investigate the effects of intraspecific competition intensity for each species. The intraspecific $(0: 20,20: 0)$ microcosms were used in both analyses. Three larval carcass inputs (control-0, medium-5, and large-10) were added to each of the five species ratios every other day for a combined 15 treatments, each treatment had 4 replicates. The experimental containers were housed in an environmental chamber at $28-30^{\circ} \mathrm{C}$ with a 14:10 light:dark photoperiod, and were checked daily for pupal emergence and mortality over the entire duration of the experiment. When pupae emerged they were removed from their microcosm, development time was recorded, and sex was identified using a dissecting microscope. Pupae were placed into $1.5 \mathrm{~mL}$ micro-centrifuge tubes and stored at $-80^{\circ} \mathrm{C}$ for later analysis. Once all larvae had either emerged as pupae or died, all pupae were thawed and cephalothorax length was measured to the nearest $1 / 100^{\text {th }} \mathrm{mm}$ using a digital caliper. Pupae collected from mixed species microcosms were identified to species using a PCR assay (Patsoula et al. 2006).

Statistical Analysis. The data was analyzed using two models to address the effect of competition-type (inter-vs intraspecific) and intraspecific competition intensity on development time, cephalothorax length, and survivorship in larval microcosms where larval detritus was the primary resource. A generalized linear mixed model (SAS 9.3 
PROC GLIMMIX), using the Satterwaithe degrees of freedom approximation to account for unequal sample sizes to analyze the continuous response variables development time and cephalothorax length and the binomial response variable survivorship. In order to graph the binomial response variable survivorship, we used PROC FREQ, SAS 9.3 to determine binomial proportions and exact $95 \%$ confidence intervals. Each model was run separately by mosquito species. For all models significant main effects and interactions were followed by pairwise contrasts of least-square means using the Bonferroni adjustment for multiple comparisons. Non-significant interactions involving sex were dropped from the models, but due to our a-priori hypotheses, non-significant interactions involving competition-type and competition intensity were kept in the models.

Competition-type model. This model yields a direct comparison between larvae that developed under intraspecific Ae. aegypti:Ae. albopictus (20:0, 0:20) competition with larvae that developed under interspecific competition (10:10). To estimate the effect of competition type on development time and cephalothorax size we assessed the dependence of the continuous variables development time and cephalothorax length on the fixed predictor variables sex, resource input, competition type and their interactions for each species. To determine larval survivorship we assessed the dependence of the binomial response variable pupal status on the fixed predicator variables resource input, competition type and their interactions. Sex was excluded in the survivorship model because the sex of mosquito larvae is indeterminate.

Competition intensity model. This model yields a direct comparison between larvae that developed under intraspecific competition in microcosms with a high or low larval density (starting ratios 10:0 vs. 20:0 and 0:10 vs. 0:20). The model structure for the 
competition intensity analysis model is identical to the competition type model, but substitutes competition intensity (high or low) for competition-type (inter or intraspecific).

\section{Competition type model}

\section{RESULTS}

We found that overall, competitive interactions between Ae. aegypti and Ae. albopictus significantly influenced cephalothorax length; Ae. aegypti from mixed species microcosms had a significantly smaller cephalothorax length, relative to Ae. aegypti from single-species microcosms. Conversely, Ae. albopictus larvae from mixed species microcosms had a significantly longer cephalothorax than Ae. albopictus from singlespecies microcosms. This difference between the species indicates that when larval detritus is the primary resource within the larval habitat, Ae. albopictus is the superior competitor relative to Ae. aegypti (Table 7, Fig. 12). However this difference was inputlevel specific: not only did increased detritus inputs led to a larger cephalothorax length in both species, it also eliminated the difference between competition types in microcosms (Table 7, Fig. 12). Male larvae were significantly smaller than female larvae for both species (data not shown) and results from a sexual dimorphism among mosquitoes (Clements 1992).

Table 7. Mixed model analysis of variance statistics for the model describing predictors of cephalothorax length. This analysis uses maximum likelihood methods to calculate approximate F-statistics. Stars indicate level of statistical significance $(*<0.05, * *<0.01$, $* * *<0.001)$.

\begin{tabular}{|c|c|c|c|c|}
\hline \multirow{2}{*}{ Source } & \multicolumn{4}{|c|}{ Cephalothorax Length } \\
\hline & Num DF & Den DF & F Value & Pr $>$ F \\
\hline \multicolumn{5}{|l|}{ Ae. albopictus } \\
\hline sex & 1 & 134.6 & 34.12 & $\star * *$ \\
\hline detritus input & 2 & 134.9 & 28.74 & $\star \star \star *$ \\
\hline competition type & 1 & 134.8 & 5.75 & $\star \star$ \\
\hline input* competition & 2 & 134.6 & 4.07 & $\star \star$ \\
\hline \multicolumn{5}{|l|}{ Ae. aegypti } \\
\hline sex & 1 & 98.02 & 8.62 & $\star *$ \\
\hline detritus input & 2 & 98.05 & 8.7 & $\star \star \star \star$ \\
\hline competition type & 1 & 97.06 & 5.97 & $\star \star$ \\
\hline input* competition & 2 & 98.53 & 7.52 & $\star \star \star$ \\
\hline
\end{tabular}


A

Ae. aegypti

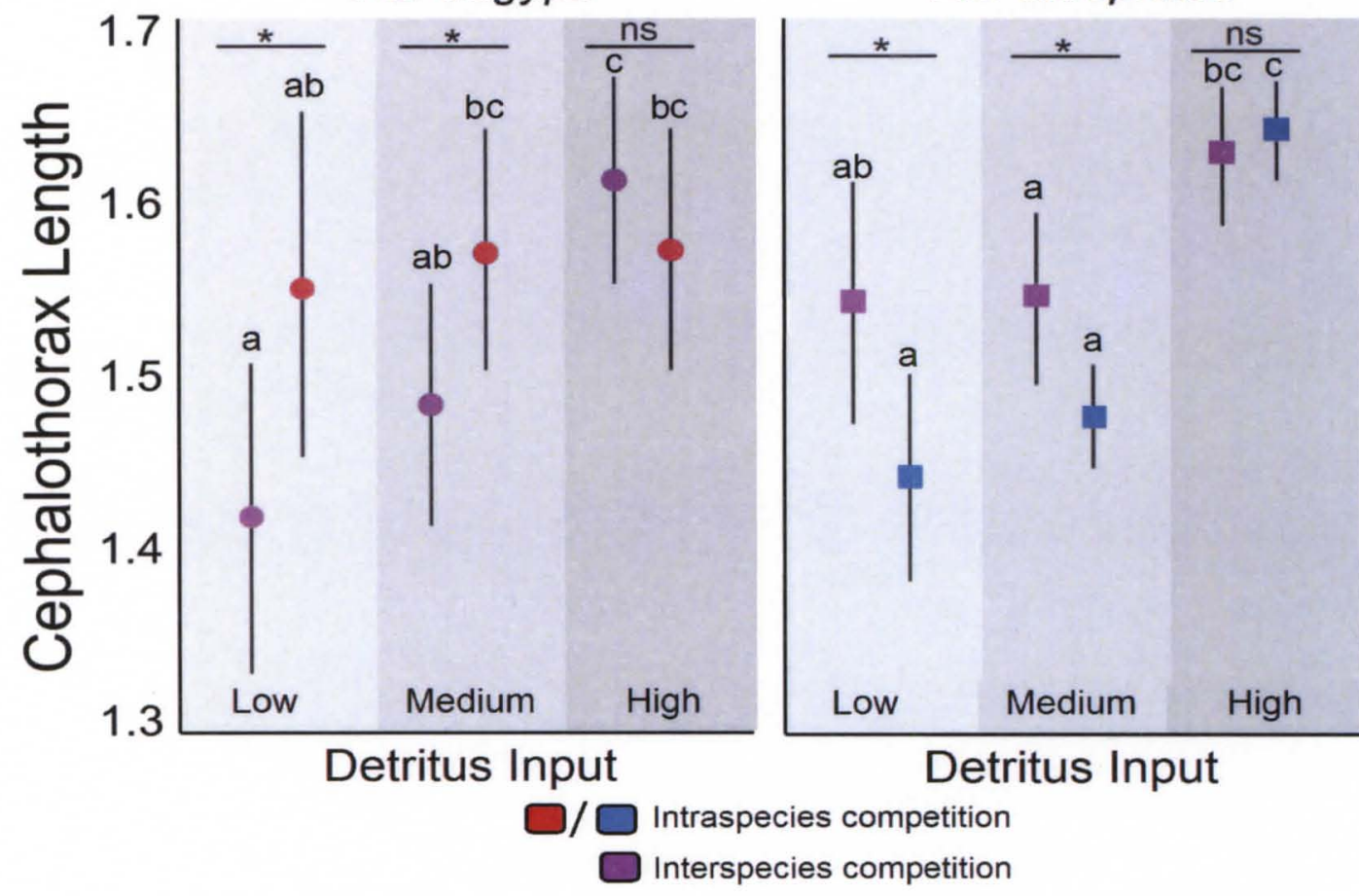

Figure 12. Input $x$ Competition-type interaction for cephalothorax length.

Cephalothorax length least-square means with $95 \%$ confidence intervals for (A) Ae. aegypti or (B) Ae. albopictus. The stars over the estimates indicate the significance level $(*<0.05$, $* *<0.01, * * *<0.001$ ) of pairwise comparisons of species ratios at each resource input from a mixed linear model with sex, detritus input, competition type, and their interactions as predictor variables, and cephalothorax length as the response variable, before controlling for multiple comparisons. For each species, estimates with a different letter are significantly different at $\mathrm{p}=0.05$, after controlling for an overall alpha across multiple comparisons of 0.05 .

In contrast to the effects on cephalothorax size, the effect of competition-type on development time was species-specific (Table 8). The development time of Ae. aegypti was not significantly influenced by competition-type, whereas competition-type influenced Ae. albopictus development time (Table 8, Fig. 13). Ae. albopictus development time was significantly shorter in interspecific competition microcosms relative to intraspecific competition microcosms, further suggesting that the outcome of interspecific competition was asymmetric and that Ae. albopictus was the superior competitor. There was a significant sex by input interaction for Ae. aegypti but not Ae. 
albopictus, though male larvae of both species developed significantly faster than

females. Male Ae. aegypti development time was positively correlated with detritus input, whereas the development time of female Ae. aegypti, and male and female Ae. albopictus larvae of was negatively correlated with detritus inputs, though no pairwise contrasts were significant after correcting for multiple comparisons (Fig 14). Increased detritus inputs significantly decreased the development time of Ae. albopictus but not Ae. aegypti (Table 8, Fig 14).

Table 8. Mixed model analysis of variance statistics for the model describing predictors of development time. Stars indicate level of statistical significance $(*<0.05, * * 0.01$, $* * *<0.001)$.

\begin{tabular}{|c|c|c|c|c|}
\hline \multirow{2}{*}{ Source } & \multicolumn{4}{|c|}{ Development Time } \\
\hline & Num DF & Den DF & F Value & $\mathrm{Pr}>\mathrm{F}$ \\
\hline \multicolumn{5}{|l|}{ Ae. albopictus } \\
\hline sex & 1 & 134.3 & 7.89 & ** \\
\hline detritus input & 2 & 135.1 & 6.35 & ** \\
\hline competition type & 1 & 134.5 & 8.13 & ** \\
\hline sex*detritus input & 2 & 134.7 & 0.35 & ns \\
\hline \multicolumn{5}{|l|}{ Ae. aegypti } \\
\hline sex & 1 & 101.8 & 8.43 & $* *$ \\
\hline detritus input & 2 & 101.8 & 0.27 & ns \\
\hline competition type & 1 & 99.9 & 0.01 & ns \\
\hline sex*detritus input & 2 & 102.6 & 3.01 & * \\
\hline
\end{tabular}

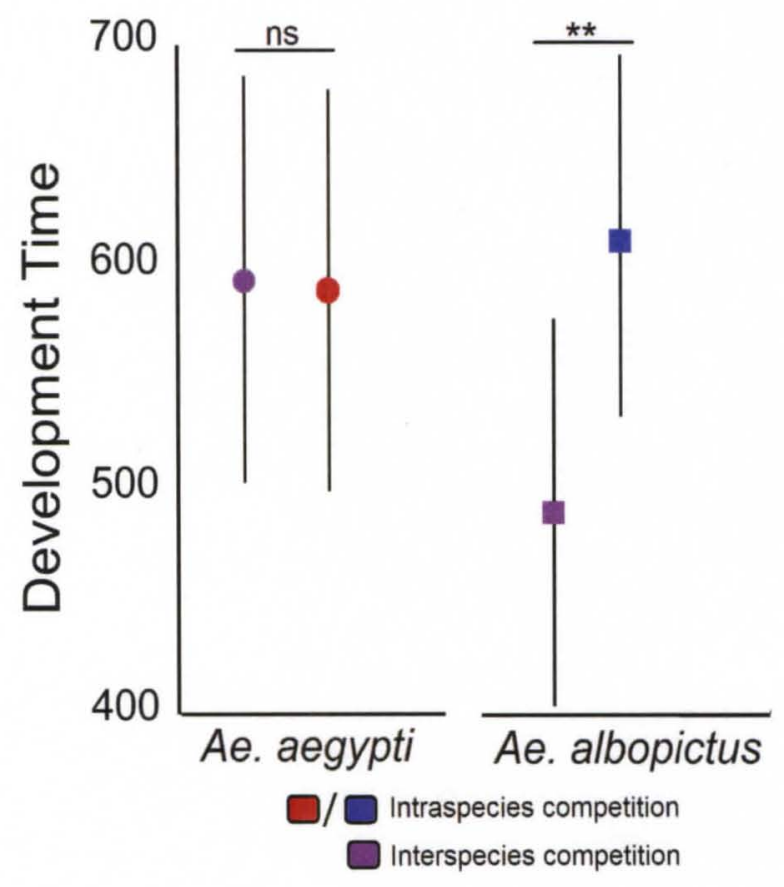

Figure 13. Effect of competition type on development time. Development time least-square means with $95 \%$ confidence intervals for (Ae. aegypti or Ae. albopictus. The stars over the estimates indicate the significance level $(*<0.05, * *<0.01, * * *<0.001)$ of pairwise comparisons of species ratios from a mixed linear model with sex, detritus input, competition type, and their interactions as predictor variables, and development time as the response variable. 


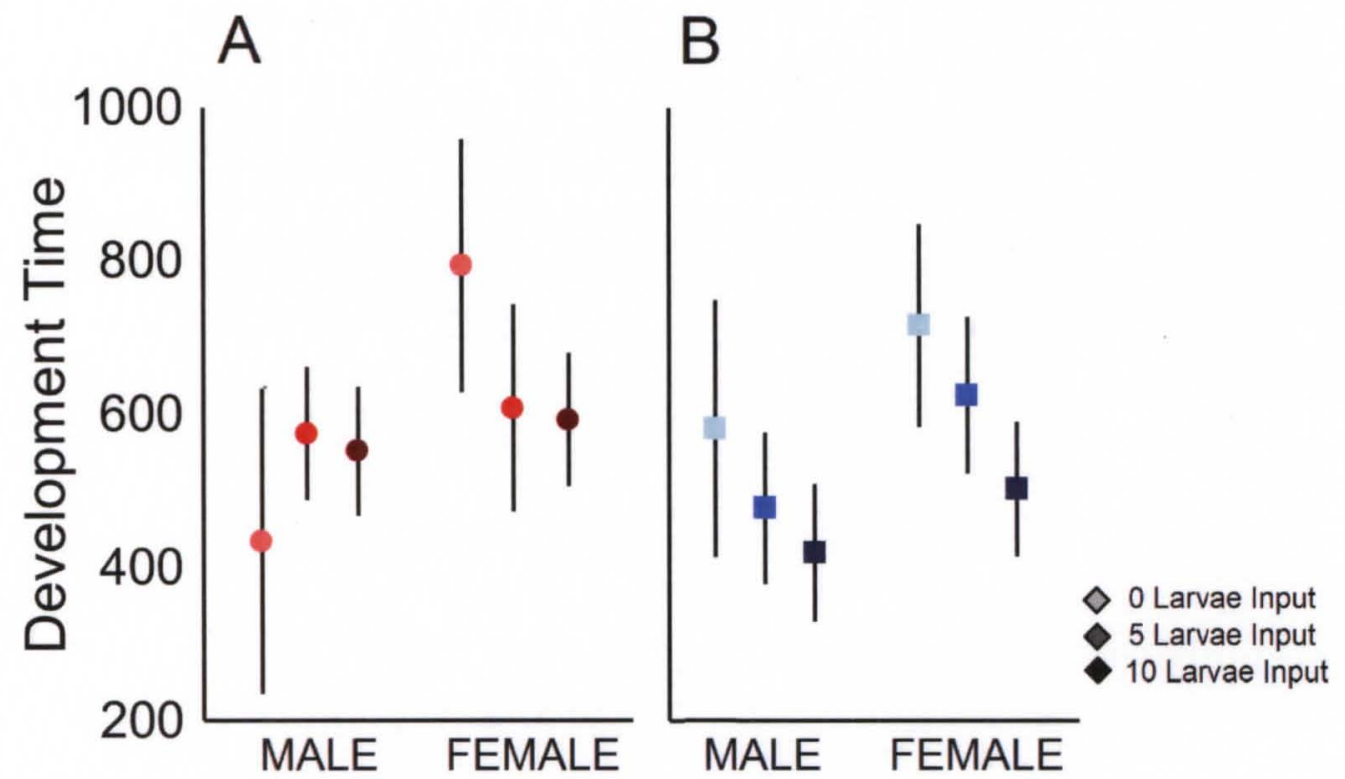

Figure 14. Sex by input interaction for development time. Development time least-square means with 95\% confidence intervals for (A) Ae. aegypti or (B) Ae. albopictus.

Competition-type significantly influenced the survivorship of Ae. aegypti but not Ae. albopictus larvae. Ae. aegypti larvae that developed in mixed-species microcosms had a significantly higher survivorship rate relative to Ae. aegypti larvae from intraspecific microcosms, while the survivorship of Ae. albopictus larvae from interspecific and intraspecific microcosms was nearly identical (Table 9, Fig. 15). Survivorship of both species was positively correlated with increased detritus inputs (Table 9).

Table 9. Mixed model analysis of variance statistics for the model describing predictors of larval survivorship. Stars indicate level of statistical significance $(*<0.05, * *<0.01$, $* * *<0.001$ ).

\begin{tabular}{|lcccc|}
\hline \multirow{2}{*}{ Source } & \multicolumn{5}{c|}{ Survivorship } \\
\cline { 2 - 5 } & Num DF & Den DF & F Value & Pr > F \\
\hline Ae. albopictus & 2 & 340 & 25.22 & $* * *$ \\
detritus input & 1 & 340 & 0.46 & $\mathrm{~ns}$ \\
\hline competition type & 2 & 340 & 1.1 & $\mathrm{~ns}$ \\
\hline competition*input & 2 & & & \\
\hline Ae. aegypti & 2 & 340 & 25.15 & $* * *$ \\
detritus input & 1 & 340 & 5.17 & $*$ \\
\hline competition type & 2 & 340 & 1.76 & $\mathrm{~ns}$ \\
\hline competition*input & 2 & &
\end{tabular}




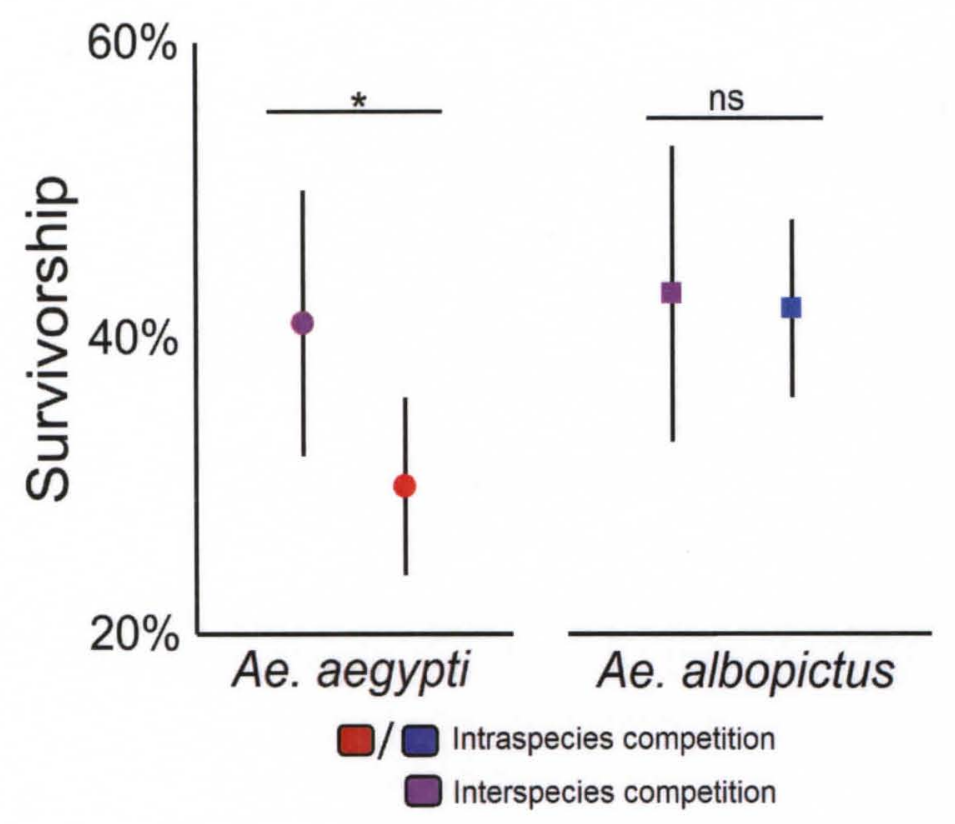

Figure 15. Effect of competition type on larval survivorship. Larval survivorship means with $95 \%$ confidence intervals for $A e$. aegypti or Ae. albopictus. The stars over the estimates indicate the significance level $(*<0.05$, $* *<0.01, * * *<0.001)$ of pairwise comparisons of species ratios from a mixed linear model with detritus input, competition type, and their interactions as predictor variables, and survivorship as the response variable.

\section{Intraspecific competition intensity model}

Overall, emergent pupae from the high-density microcosms were significantly smaller than pupae from the low-density microcosms, but the effect of density across levels of detritus input differed between Ae. aegypti and Ae. albopictus. In the high larval density microcosms, increased levels of larval detritus inputs did not result in the increased cephalothorax length of Ae. aegypti, whereas the cephalothorax length of Ae.

albopictus was highly correlated with the level of detritus input (Table 10, Fig. 16).

Table 10. Mixed model analysis of variance statistics for the model describing predictors of cephalothorax length. Stars indicate level of statistical significance $\left(*<0.05,{ }^{*}<0.01\right.$, $* * *<0.001)$.

\begin{tabular}{|c|c|c|c|c|}
\hline \multirow{2}{*}{ Source } & \multicolumn{4}{|c|}{ Cephalothorax Length } \\
\hline & Num DF & Den DF & F Value & Pr $>$ F \\
\hline \multicolumn{5}{|l|}{ Ae. albopictus } \\
\hline sex & 1 & 168 & 73.6 & $* * *$ \\
\hline detritus input & 2 & 168 & 38.1 & $* * *$ \\
\hline density & 1 & 168 & 61.81 & $* \star \star$ \\
\hline sex*input & 2 & 168 & 1.31 & ns \\
\hline density*input & 2 & 168 & 8.31 & $\star \star *$ \\
\hline \multicolumn{5}{|l|}{ Ae. aegypti } \\
\hline sex & 1 & 131.7 & 29.91 & $\star \star \star \star$ \\
\hline detritus input & 2 & 131.3 & 4.59 & $\star \star$ \\
\hline density & 1 & 131.6 & 6.89 & $* \star \star \star$ \\
\hline sex*input & 2 & 131 & 3.75 & $\star \star$ \\
\hline density*input & 2 & 131.4 & 3.03 & * \\
\hline
\end{tabular}




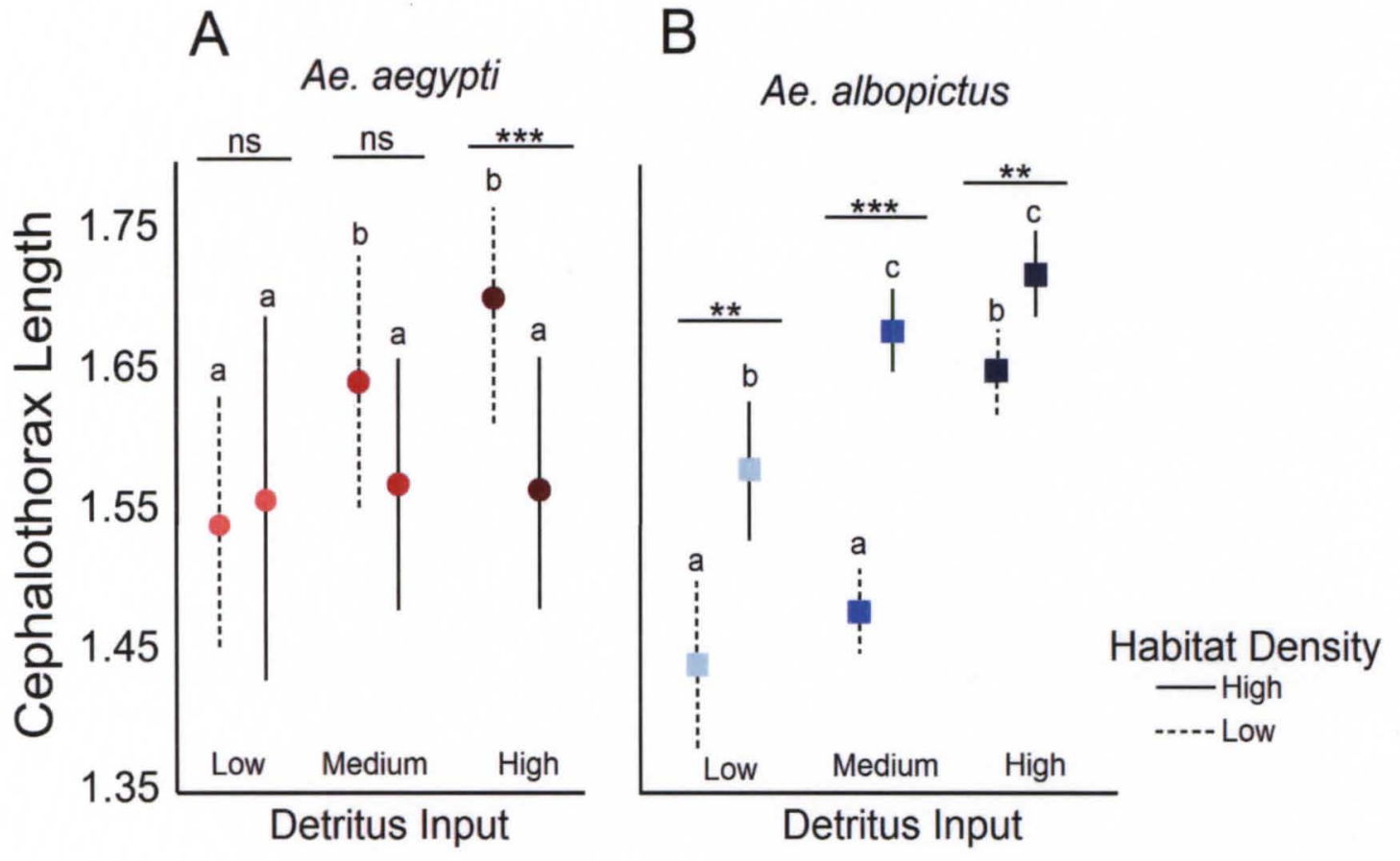

Figure 16. Input $x$ Competition intensity interaction for cephalothorax length.

Cephalothorax length least-square means with $95 \%$ confidence intervals for (A) Ae. aegypti or (B) Ae. albopictus. The stars over the estimates indicate the significance level $(*<0.05$, $* *<0.01, * * *<0.001)$ of pairwise comparisons at each species ratio from a mixed linear model with sex, detritus input, competition intensity, and their interactions as predictor variables, and cephalothorax length as the response variable. For each species, estimates with a different letter are significantly different at $\mathrm{p}=0.05$, after controlling for an overall alpha across multiple comparisons of 0.05 .

For Ae aegypti, there was a significant sex by input interaction (Table 10). The cephalothorax length of female larvae significantly increased in size as detritus inputs increased, while there was no significant effect of detritus input on male cephalothorax length (data not shown).

In addition to resulting in smaller cephalothorax length, increasing competition intensity resulted overall in an extension of development time (Table 11, Fig. 17). Overall, increased larval detritus inputs decreased development time for both species. There was also a significant input by density interaction, whereas Ae, aegypti larvae 
receiving the low and medium detritus input developed at the same rate, at high detritus input, decreasing competition intensity resulted in faster development (Fig 17).

Table 11. Mixed model analysis of variance statistics for the model describing predictors of development time. Stars indicate level of statistical significance $(*<0.05, * *<0.01$, $* * *<0.001)$.

\begin{tabular}{|lcccc|}
\hline \multirow{2}{*}{ Source } & \multicolumn{4}{c|}{ Development Time } \\
\cline { 2 - 5 } & Num DF & Den DF & F Value & Pr $>$ F \\
\hline Ae. albopictus & 1 & 167.9 & 7.02 & $* *$ \\
sex & 2 & 168 & 18.5 & $* * *$ \\
\hline detritus input & 1 & 169.4 & 31.4 & $* * *$ \\
\hline density & 2 & 168.7 & 2.18 & ns \\
\hline density*input & & & & \\
\hline Ae. aegypti & 1 & 136.6 & 15.5 & $* \star$ \\
sex & 2 & 136.4 & 5.5 & $* *$ \\
\hline detritus input & 1 & 136.1 & 3.7 & $*$ \\
\hline density & 2 & 136.4 & 4.1 & $* *$ \\
\hline density*input & 2 & & & \\
\hline
\end{tabular}

A

B

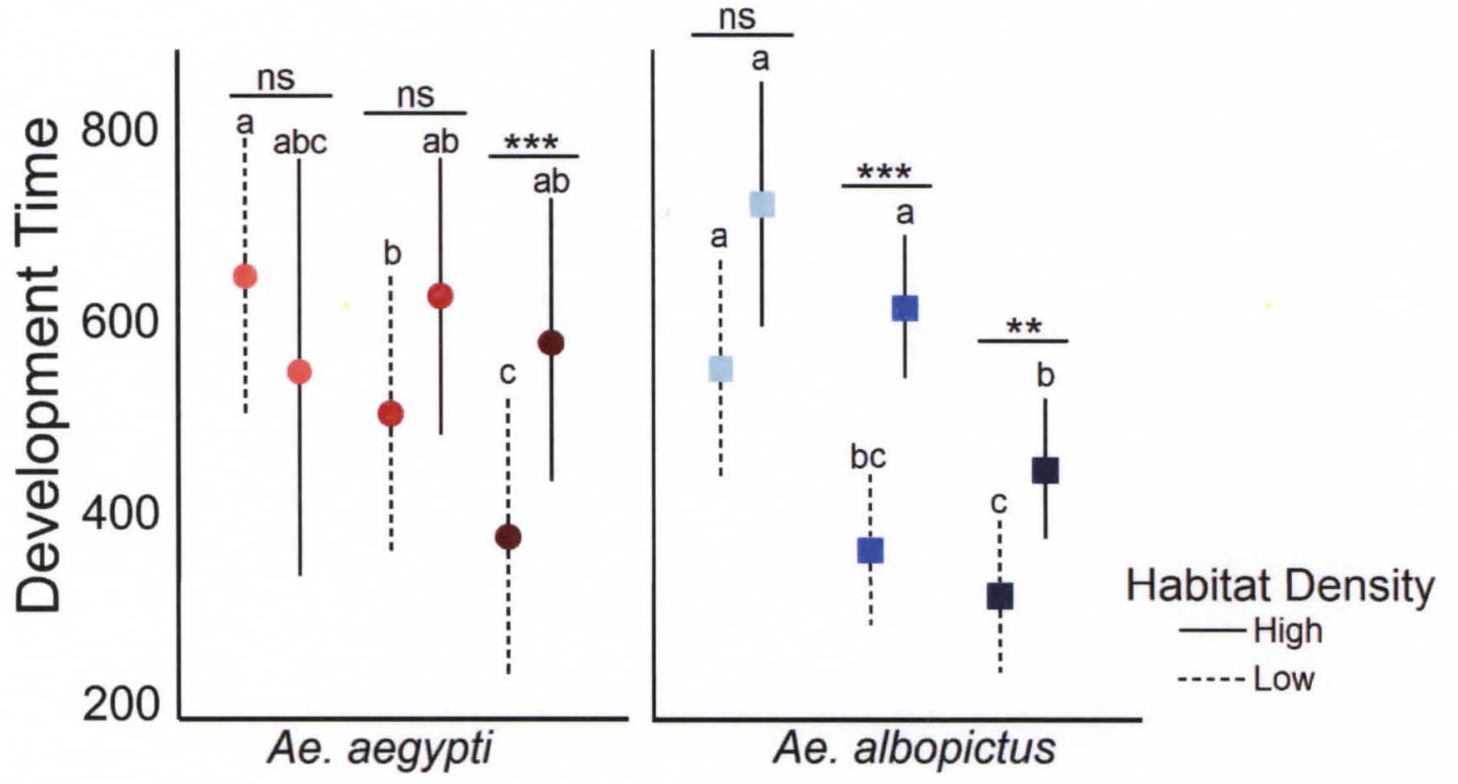

Figure 17. Input $x$ Competition intensity interaction for development time.

Cephalothorax length least-square means with $95 \%$ confidence intervals for (A) Ae. aegypti or (B) Ae. albopictus. The stars over the estimates indicate the significance level $(*<0.05$, $* *<0.01, * * * 0.001$ ) between species ratios at each resource input and results of pairwise comparisons from a mixed linear model with sex, detritus input, competition intensity, and their interactions as predictor variables, and cephalothorax length as the response variable. For each species, estimates with a different letter are significantly different at $\mathrm{p}=0.05$, after controlling for an overall alpha across multiple comparisons of 0.05 . 
Competition intensity and detritus inputs both significantly influenced larval survivorship but the density by detritus input was not significant for either Ae. aegypti or Ae. albopictus (Table 12, Fig. 18). There was a strong positive correlation between detritus input and larval survivorship (Table 12). Larval survivorship was significantly lower in high-density microcosms for both species regardless of the detritus input.

Table 12. Mixed model analysis of variance statistics for the model describing predictors of larval survivorship. Stars indicate level of statistical significance $(*<0.05, * *<0.01$, $* * *<0.001)$.

\begin{tabular}{|lcccc|}
\hline \multirow{2}{*}{ Source } & \multicolumn{4}{c|}{ Survivorship } \\
\cline { 2 - 5 } & Num DF & Den DF & F Value & Pr $>$ F \\
\hline Ae. albopictus & & & & \\
detritus input & 2 & 354 & 35.45 & $* * *$ \\
\hline density & 1 & 354 & 23.6 & $* * *$ \\
\hline density*input & 2 & 354 & 0.12 & ns \\
\hline Ae. aegypti & & & & \\
detritus input & 2 & 355 & 20.2 & $* * *$ \\
\hline density & 1 & 355 & 51.1 & $* * *$ \\
\hline density*input & 2 & 355 & 1.76 & ns \\
\hline
\end{tabular}
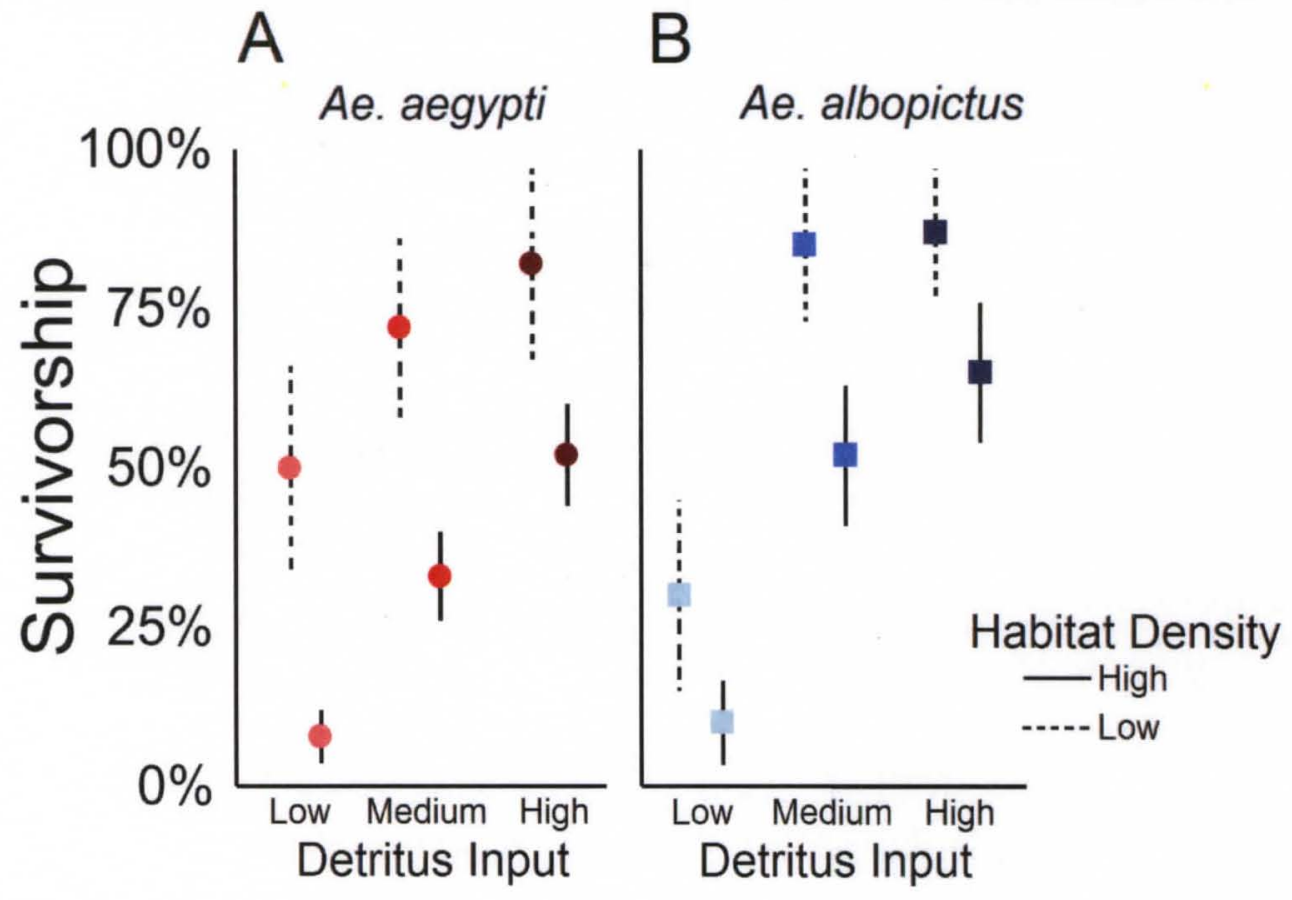

Figure 18. Effect of competition intensity and detritus input on larval survivorship. Mean survivorship with $95 \%$ confidence intervals for (A) Ae. aegypti or (B) Ae albopictus. 


\section{DISCUSSION}

Resource competition within the larval habitat has an important role in shaping community structure and can influence both epidemiologically important adult life history traits, and the geographic distribution of mosquito species (Juliano and Lounibos 2005, Reiskind and Lounibos 2009, Alto 2011). The objective of this experiment was to determine the effect of interspecific competition and intraspecific competition intensity on larval Ae. aegypti and Ae. albopictus. Since interspecific competitive outcomes are usually context-dependent, we used a plant detritus source, Shumard red oak, which is common in urban environments throughout the shared range of Ae. albopictus and Ae. aegypti within the Southeastern US, and added larval carcasses which are a form of invertebrate detritus found within all larval habitats.

\section{Competition Type}

We found that the outcome of interspecific competition between Ae. aegypti and Ae. albopictus was asymmetric; Ae. albopictus significantly outcompeted Ae. aegypti within mixed-species microcosms. Ae. albopictus from interspecific microcosms were significantly larger and developed faster than Ae. albopictus larvae from intraspecific microcosms (Fig 12). There was no effect of competition-type on Ae. albopictus survivorship (Fig 15). The opposite was true regarding the cephalothorax length of $A e$. aegypti, where larvae reared from intraspecific microcosms were significantly larger. Competition-type did not significantly influence Ae. aegypti development time (Fig 13). Despite the fact the Ae. albopictus was the superior competitor, Ae. aegypti survivorship 
was higher among larvae reared in mixed-species microcosms compared to single species microcosms (Fig 15).

Of particular importance is the effect of interspecific competition on cephalothorax length (Fig 12), which is highly correlated with adult life span and susceptibility to arboviruses (Fig 2) (Alto et al. 2008). Smaller adults are generally more susceptible to arboviruses than large adults but have a decreased life span that reduces the likelihood of surviving the extrinsic incubation period and successfully transmitting the virus (Fig 3)(Grimstad and Walker 1991). While it is not yet clear whether small or large mosquitoes are more efficient vectors, our results suggest that the vectorial capacity of both Ae. aegypti and Ae. albopictus populations may be altered as a result of interspecific competition when larval detritus is the primary resource input.

In addition to influencing adult life history traits, competitive asymmetry can also lead to competitive displacement of mosquito species across the shared host range (Juliano and Lounibos 2005). We found that while the presence of interspecific competition did not significantly reduce the survivorship of Ae. aegypti larvae, the expected reduction in Ae. aegypti population size due to reduced fecundity and decreased survivorship as a result of smaller adult body size could over time lead to the displacement of Ae. aegypti. Since the arrival of Ae. albopictus in the US in 1985, it has rapidly expanded its geographic range throughout the Southeast US and displaced $A e$. aegypti across most of its established range (Hawley 1988, Rey et al. 2006). Understanding the outcome of context-dependent interspecific interactions can provide important information regarding the potential for future range expansion of invasive species and the associated risk of vector-borne disease transmission. Ae. aegypti is a 
more effective vector of many arboviruses relative to Ae. albopictus and therefore its displacement throughout the Southeast United States may have reduced the threat of dengue transmission.

\section{Intraspecific Competition Intensity}

Overall, intraspecific competition intensity affected Ae. aegypti and $A e$. albopictus differently. Among Ae. albopictus there was a much stronger response to both detritus input and larval density. While, among Ae. aegypti the effect of density and detritus input on survivorship and development were density and sex-dependent; at high density increased detritus inputs did not result in a larger cephalothorax length (Fig 16) or decreased development time (Fig 17) but did increase survivorship (Fig 18).

There have been a number of studies that have investigated the outcome of competitive interactions (Juliano 2009), few if any studies have investigated the possibility of sex-dependent responses to resource levels, competition-type, or competition intensity, as we have in this study. While we found no competition-type by sex interaction, we detected two significant sex by input interactions involving Ae. aegypti. Pairwise contrasts indicate both interactions were driven by the inverse effect of detritus input on male larvae for development time (Table 8) and cephalothorax length (Table 10). Increasing the level of detritus input resulted in an increase in development time (Fig 14) or a decrease in cephalothorax length (data not shown). This indicates that there may be a trade-off between resource inputs and competition intensity, which may affect the development of both species and sexes differently. One significant implication of this result is that it suggests that under certain detrital conditions, the effect of competition intensity may also be context-dependent and sex-dependent. 
In this study we assessed the competitive interactions among and between Ae. aegypti and Ae.albopictus larvae using a type of resource, larval detritus, that had never been investigated previously but is common within larval habitats. The results from our interspecific competition experiment are consistent with previous studies that investigated the outcome of interspecific competition between Ae. aegypti and Ae. albopictus using different forms of invertebrate detritus, indicating that there is likely a physiological mechanism underlying the competitive superiority of $A e$. albopictus across a broad range of detritus sources (Daughtery et al. 2000, Braks et al. 2004, Murrell and Juliano 2009). 


\section{REFERENCES}

Adams, B., \& Boots, M. (2010). How important is vertical transmission in mosquitoes for the persistence of dengue? Insights from a mathematical model. Epidemics, 2, 1 10.

Alizon, S., Hurford, A., Mideo, N., \& van Baalen, M. (2008). Virulence evolution and the trade-off hypothesis: history, current state of affairs and the future. Journal of Evolutionary Biology, 22, 245-259.

Almeida, A. P. G., Baptista, S. S. S. G., Sousa, C. A. G. C. C., Novo, M. T. L. M., Ramos, H. C., Panella, N. A., Godsey, M., et al. (2005). Bioecology and Vectorial Capacity of Aedes albopictus(Diptera: Culicidae) in Macao, China, in Relation to Dengue Virus Transmission. Journal of Medical Entomology, 42, 419-428.

Alto, B. W., Lounibos, L. P., Higgs, S., \& Juliano, S. A. (2005). Larval competition differentially affects arbovirus infection in Aedes mosquitoes. Ecology, 86, 3279_ 3288.

Alto, B. W., Lounibos, L. P., Mores, C. N., \& Reiskind, M. H. (2008A). Larval competition alters susceptibility of adult Aedes mosquitoes to dengue infection. Proceedings of the Royal Society B: Biological Sciences, 275, 463-471.

Alto, B. W., Reiskind, M. H., \& Lounibos, L. P. (2008B). Size Alters Susceptibility of Vectors to Dengue Virus Infection and Dissemination. The American Journal of 
Tropical Medicine and Hygiene, 79, 688-695.

Alto, B.W. (2011). Interspecific larval competition between invasive Aedes japonicas and native Aedes triseriatus (Diptera: Culicidae) and adult longevity. Journal of Medical Entomology, 48, 232-242.

Anderson, J. R., \& Rico-Hesse, R. (2006). Aedes aegypti vectorial capacity is determined by the infecting genotype of dengue virus. American Journal of Tropical Medicine and Hygiene, 75, 886-892.

André, J. B., \& Hochberg, M. E. (2005). Virulence evolution in emerging infectious diseases. Evolution, 59, 1406-1412.

Angel, B., \& Joshi, V. (2008). Distribution and seasonality of vertically transmitted dengue viruses in Aedes mosquitoes in arid and semi-arid areas of Rajasthan, India. Journal of Vector Borne Diseases, 45, 56-59.

Angel, B., Sharma, K., \& Joshi, V. (2008). Association of ovarian proteins with transovarial transmission of dengue viruses by Aedes mosquitoes in Rajasthan, India. The Indian Journal of Medical Research, 128, 320-323.

Armbruster, P., \& Conn, J. E. (2006). Geographic variation of larval growth in North American Aedes albopictus (Diptera: Culicidae). Annals of the Entomological Society of America, 99, 1234-1243.

Armstrong, P. M., \& Rico-Hesse, R. (2003). Efficiency of dengue serotype 2 virus strains to infect and disseminate in Aedes aegypti. American Journal of Tropical Medicine and Hygiene, 68, 539-544.

Arrivillaga, J., \& Barrera, R. (2004). Food as a limiting factor for Aedes aegypti in waterstorage containers. Journal of Vector Ecology, 29, 11-20. 
Bevins, S. N. (2007A). Invasive mosquitoes, larval competition, and indirect effects on the vector competence of native mosquito species (Diptera: Culicidae). Biological Invasions, 10, 1109-1117.

Bevins, S. N. (2007B). Timing of resource input and larval competition between invasive and native container-inhabiting mosquitoes (Diptera: Culicidae). Journal of Vector Ecology, 32, 252-262.

Black, W. C., Bennett, K. E., Gorrochótegui-Escalante, N., Barillas-Mury, C. V., Fernández-Salas, I., de Lourdes Muñoz, M., Farfán-Alé, J. A., et al. (2002). Flavivirus susceptibility in Aedes aegypti. Archives of Medical Research, 33, 379388.

Bosio, C. F., Thomas, R. E., Grimstad, P. R., \& Rai, K. S. (1992). Variation in the efficiency of vertical transmission of dengue-1 virus by strains of Aedes albopictus (Diptera: Culicidae). Journal of Medical Entomology, 29, 985-989.

Braks, M. A. H., Honorio, N.A., Lounibos, L.P., Lourenco-De-Oliveria, R., \& Juliano, S.A. (2004). Interspecific competition between two invasive species of container mosquitoes, Aedes aegypti and Aedes albopictus (Diptera:Culicidae), in Brazil. Annals of the Entomological Society of America, 97, 130-139

Briegel, H., \& Timmermann, S. E. (2001). Aedes albopictus (Diptera: Culicidae): physiological aspects of development and reproduction. Journal of Medical Entomology, 38, 566-571.

Briegel, H., Knüsel, I., \& Timmermann, S. E. (2001). Aedes aegypti: size, reserves, survival, and flight potential. Journal of Vector Ecology, 26, 21-31.

Castro, M. G., Nogueira, R. M. R., Schatzmayr, H. G., Miagostovich, M. P., \& Lourenço- 
de-Oliveira, R. (2004). Dengue virus detection by using reverse transcriptionpolymerase chain reaction in saliva and progeny of experimentally infected Aedes albopictus from Brazil. Memórias do Instituto Oswaldo Cruz, 99, 809-814.

Cecílio, A. B., Campanelli, E. S., Souza, K. P. R., Figueiredo, L. B., \& Resende, M. C. (2009). Natural vertical transmission by Stegomyia albopicta as dengue vector in Brazil. Brazilian Journal of Biology, 69, 123-127.

Chambers, T.J., Hahn, C.S., Galler, R., \& Rice, C.M. (1990). Flavivirus genome organization, expression, and replication. Annual Review of Microbiology, 44, 649688.

Chaudhry, S., Swaminathan, S., Khanna, N. (2006). Viral genetics as a basis of dengue pathogenesis. Dengue Bulletin. 30, 121-132.

Chen, R., \& Vasilakis, N. (2011). Dengue - Quo tu et quo vadis? Viruses, 3, 1562-1608.

Choochote, W., Tippawangkosol, P., Jitpakdi, A. Sukontason, K. L., Pitasawat, B., Sukontason, K. \& Jaripyapan, N. (2001). Polygamy: the possibly significant behavior of Aedes aegypti and Aedes albopictus in relation to the efficient transmission of dengue virus. Southeast Asian Journal of Tropical Medicine and Public Health. 32, 745-748.

Clements, A.N. (1992). The Biology of Mosquitoes Volume 1; Development, Nutrition, and Reproduction. New York: CABI Publishing Company.

Clyde, K., Kyle, J. L., \& Harris, E. (2006). Recent Advances in Deciphering Viral and Host Determinants of Dengue Virus Replication and Pathogenesis. Journal of Virology, 80, 11418-11431.

Collins, W. E. (1962). Trans-stadial passage of St. Louis encephalitis virus in Aedes 
aegypti mosquitoes. American Journal of Tropical Medicine and Hygiene, 11, $535-538$.

Cologna, R., \& Rico-Hesse, R. (2003). American genotype structures decrease dengue virus output from human monocytes and dendritic cells. Journal of Virology, 77, 3929.

Cologna, R., Armstrong, P. M., \& Rico-Hesse, R. (2005). Selection for virulent dengue viruses occurs in humans and mosquitoes. Journal of Virology, 79, 853-859.

Costanzo, K. S., Muturi, E. J., Lampman, R. L., \& Alto, R. W. (2011). The Effects of Resource Type and Ratio on Competition with Aedes albopictus and Culex pipiens (Diptera: Culicidae). Journal of Medical Entomology, 48, 29-38.

Daugherty, M. P., Alto, B. W., \& Juliano, S. A. (2000). Invertebrate carcasses as a resource for competing Aedes albopictus and Aedes aegypti (Diptera: Culicidae). Journal of Medical Entomology, 37, 364-372.

De Castro, MC., Nogueira, R.M.R., Schatzmayr, H.G., Miagostovich, M.P. \& Lourençode-Oliveira, R. (2004). Dengue virus detection by using reverse transcriptionpolymerase chain reaction in saliva and progeny of experimentally infected Aedes albopictus from Brazil. Mem. Inst. Oswaldo Cruz, 99, 809-814.

Diallo, M., Ba, Y., Faye, O., Soumare, M. L., Dia, I., \& Sall, A. A. (2008). Vector competence of Aedes aegypti populations from Senegal for sylvatic and epidemic dengue 2 virus isolated in West Africa. Transactions of the Royal Society of Tropical Medicine and Hygiene, 102, 493-498.

Diallo, M., Sall, A. A., Moncayo, A. C., Ba, Y., Fernandez, Z., Ortiz, D., Coffey, L. L., et al. (2005). Potential role of sylvatic and domestic African mosquito species in 
dengue emergence. American Journal of Tropical Medicine and Hygiene, 73, 445449.

Dodson, B. L., Kramer, L. D., \& Rasgon, J. L. (2012). Larval Nutritional Stress Does Not Affect Vector Competence for West Nile Virus (WNV) in Culex tarsalis. VectorBorne and Zoonotic Diseases, 1-6.

Dye, C. (1986). Vectorial capacity:must we measure all its components? Parasitology Today, 2, 203-209.

Elliot, S.L., Adler, F.R., \& Sabelis, M.W. (2003). How Virulent Should A Parasite Be To Its Vector? Ecology, 84, 2568-2574.

Esteva, L., \& Vargas, C. (2000). Influence of vertical and mechanical transmission on the dynamics of dengue disease. Mathematical Biosciences, 167, 51-64.

Faran, M. E., Turell, M. J., Romoser, W. S., Routier, R. G., Gibbs, P. H., Cannon, T. L., \& Bailey, C. L. (1987). Reduced survival of adult Culex pipiens infected with Rift Valley fever virus. American Journal of Tropical Medicine and Hygiene, 37, 403409.

de Figueiredo, M. L., de C Gomes, A., Amarilla, A. A., de S Leandro, A., de S Orrico, A., de Araujo, R. F., do SM Castro, J., et al. (2010). Mosquitoes infected with dengue viruses in Brazil. Virology Journal, 7(1), 152.

Ferguson, H. M., \& Read, A. F. (2002). Why is the effect of malaria parasites on mosquito survival still unresolved? Trends in parasitology, 18, 256-261.

Fish, D., \& Carpenter, S. R. (1982). Leaf litter and larval mosquito dynamics in tree-hole ecosystems. Ecology, 283-288.

Gaunt, M. W., Sall, A. A., de Lamballerie, X., Falconar, A. K., Dzhivanian, T. I., \& 
Gould, E. A. (2001). Phylogenetic relationships of flaviviruses correlate with their epidemiology, disease association and biogeography. The Journal of general virology, 82(Pt 8), 1867-1876.

Gratz, N. G. (2004). Critical review of the vector status of Aedes albopictus. Medical and veterinary entomology, 18, 215-227.

Grimstad, P. R., \& Walker, E. D. (1991). Aedes triseriatus (Diptera: Culicidae) and La Crosse virus. Nutritional deprivation of larvae affects the adult barriers to infection and transmission. Journal of Medical Entomology, 28, 378-386.

Gubler, D. J. (1998). Dengue and dengue hemorrhagic fever. Clinical Microbiology Reviews, 11, 480-496.

Gubler, D. J. (2004). Cities spawn epidemic dengue viruses. Nature medicine, 129-130.

Guo, X., Zhao, T., Dong, Y., \& Lu, B. (2007). Survival and replication of dengue-2 virus in diapausing eggs of Aedes albopictus (Diptera: Culicidae). Journal of Medical Entomology, 44, 492-497.

Guzman, A., \& Istúriz, R. E. (2010). Update on the global spread of dengue. International Journal of Antimicrobial Agents, 36, S40-S42.

Guzman, M. G., \& Kouri, G. (2002). Dengue: an update. The Lancet Infectious Diseases, $2,33-42$.

Guzman, M. G., Halstead, S. B., Artsob, H., Buchy, P., Farrar, J., Gubler, D. J., Hunsperger, E., et al. (2010). Dengue: a continuing global threat. Nature Reviews Microbiology, 8, S7-S16.

Halstead, S. B. (2007). Dengue. The Lancet, 370, 1644-1652.

Hardy, J. L., Houk, E. J., Kramer, L. D., \& Reeves, W. C. (1983). Intrinsic factors 
affecting vector competence of mosquitoes for arboviruses. Annual Review of Entomology, 28, 229-262.

Harrington, L. C., Edman, J. D., \& Scott, T. W. (2001). Why Do Female Aedes aegypti (Diptera: Culicidae) Feed Preferentially and Frequently on Human Blood? Journal of Medical Entomology, 38, 411-422.

Hawley, W.A. (1988). The biology of Aedes albopictus. Journal of the American Mosquito Control Association, S1, 1-40

Hume, J.C.C., Hamilton, H., Lee, K.L., \& Lehmann, T. (2011). Susceptibility of Anopheles stephensi to Plasmodium gallinaceum: a trait of the mosquito, the parasite, and the environment. PLoS ONE, 6, 20156.

Holmes, E., \& Twiddy, S. (2003). The origin, emergence and evolutionary genetics of dengue virus. Infection, Genetics and Evolution, 3, 19-28.

Hull, B., Tikasingh, E., de Souza, M., \& Martinez, R. (1984). Natural transovarial transmission of dengue 4 virus in Aedes aegypti in Trinidad. American Journal of Tropical Medicine and Hygiene, 33, 1248-1250.

Ibáñez-Bernal, S., Briseño, B., Mutebi, J. P., Argot, E., Rodriguez, G., Martínez-Campos, C., Paz, R., et al. (1997). First record in America of Aedes albopictus naturally infected with dengue virus during the 1995 outbreak at Reynosa, Mexico. Medical and Veterinary Entomology, 11, 305-309.

Jansen, C. C., \& Beebe, N. W. (2010). The dengue vector Aedes aegypti: what comes next. Microbes and infection 12, 272-279.

Joshi, V., Mourya, D., \& Sharma, R. (2002). Persistence of dengue-3 virus through transovarial transmission passage in successive generations of Aedes aegypti 
mosquitoes. American Journal of Tropical Medicine and Hygiene, 67, 158.

Joshi, V., Singhi, M., \& Chaudhary, R. C. (1996). Transovarial transmission of dengue 3 virus by Aedes aegypti. Transactions of the Royal Society of Tropical Medicine and Hygiene, 90, 643-644.

Juliano, S. A. (1998). Species introduction and replacement among mosquitoes: interspecific resource competition or apparent competition? Ecology, 79, 255-268.

Juliano, S. A. (2009). Species Interactions Among Larval Mosquitoes: Context Dependence Across Habitat Gradients. Annual Review of Entomology, 54, 37-56.

Juliano, S. A., \& Lounibos, L.P. (2005). Ecology of invasive mosquitoes: effects on resident species and on human health. Ecology Letters, 8, 558-574.

Juliano, S. A., Lounibos, L. P., \& O’Meara, G. F. (2004). A field test for competitive effects of Aedes albopictus on A. aegypti in South Florida: differences between sites of coexistence and exclusion? Oecologia, 139, 583-593.

Kesavaraju, B., Yee, D. A., \& Juliano, S. A. (2007). Interspecific and intraspecific differences in foraging preferences of container-dwelling mosquitoes. Journal of Medical Entomology, 44, 215.

Klowden, M. J., Blackmer, J. L., \& Chambers, G. M. (1988). Effects of larval nutrition on the host-seeking behavior of adult Aedes aegypti mosquitoes. Journal of the American Mosquito Control Association, 4, 73-75.

Koenraadt, C. J. M. (2008). Pupal dimensions as predictors of adult size in fitness studies of Aedes aegypti (Diptera: Culicidae). Journal of Medical Entomology, 45, 331336.

Kow, C. Y., Koon, L. L., \& Yin, P. F. (2001). Detection of Dengue Viruses in Field 
Caught Male Aedes aegypti and Aedes albopictus (Diptera: Culicidae) in Singapore by Type-Specific PCR. Journal of Medical Entomology, 38, 475-479.

Kramer, L. D., \& Ebel, G. D. (2003). Dynamics of flavivirus infection in mosquitoes. Advances in Virus Research, 60, 187-232.

Kuno, G. (1995). Review of the factors modulating dengue transmission. Epidemiologic reviews, 17, 321-335.

Kuno, G., \& Chang, G.J. (2005). Biological transmission of arboviruses: reexamination of and new insights into components, mechanisms, and unique traits as well as their evolutionary trends. Clinical Microbiology Reviews, 18, 608-637.

Kyle, J. L., \& Harris, E. (2008). Global Spread and Persistence of Dengue. Annual Review of Microbiology, 62, 71-92.

Lambrechts, L., Chevillion, C., Albright, R.G., Thaisomboonsuk, B., Richardson, J.H., Jarman, R.G., \& Scott, T. W. (2009). Genetic specificity and potential for local adaptation between dengue viruses and mosquito vectors. BMC Evolutionary Biology, 9, 160.

Lambrechts, L., \& Scott, T. W. (2009). Mode of transmission and the evolution of arbovirus virulence in mosquito vectors. Proceedings of the Royal Society $B$ : Biological Sciences, 276, 1369-1378.

Lambrechts, L., Scott, T. W., \& Gubler, D. J. (2010). Consequences of the Expanding Global Distribution of Aedes albopictus for Dengue Virus Transmission. PLoS Neglected Tropical Diseases, 4, e646.

Le,C.T., 1981. A new estimator for infection rates using pools of variable size. American. Journal of Epidemiology, 114, 132-136. 
Legros, M., Lloyd, A. L., Huang, Y., \& Gould, F. (2009). Density-dependent intraspecific competition in the larval stage of Aedes aegypti (Diptera: Culicidae): revisiting the current paradigm. Journal of Medical Entomology, 46(3), 409-419.

Leisnham, P. T., \& Juliano, S. A. (2009). Spatial and temporal patterns of coexistence between competing Aedes mosquitoes in urban Florida. Oecologia, 160, 343-352.

Leisnham, P. T., \& Juliano, S. A. (2010). Interpopulation differences in competitive effect and response of the mosquito Aedes aegypti and resistance to invasion by a superior competitor. Oecologia, 164, 221-230.

Leitmeyer, K. C., Vaughn, D. W., Watts, D. M., Salas, R., Villalobos, I., de Chacon, Ramos, C., et al. (1999). Dengue virus structural differences that correlate with pathogenesis. Journal of Virology, 73, 4738-4747.

Levin, B. R. (1996). The evolution and maintenance of virulence in microparasites. Emerging Infectious Diseases, 2, 93-102.

Lounibos, L. P. (2002). Invasions by insect vectors of human disease. Annual Review of Entomology, 47, 233-266.

Maciel-de-Freitas, R., Koella, J. C., \& Lourenço-de-Oliveira, R. (2011). Lower survival rate, longevity and fecundity of Aedes aegypti (Diptera: Culicidae) females orally challenged with dengue virus serotype 2. Transactions of the Royal Society of Tropical Medicine and Hygiene, 105, 452-458.

Mackenzie, J. S., Gubler, D. J., \& Petersen, L. R. (2004). Emerging flaviviruses: the spread and resurgence of Japanese encephalitis, West Nile and dengue viruses. Nature Medicine, 10, S98-S109.

Mahmood, F., Reisen, W. K., Chiles, R. E., \& Fang, Y. (2004). Western Equine 
Encephalomyelitis Virus Infection Affects the Life Table Characteristics of Culex tarsalis (Diptera: Culicidae). Journal of Medical Entomology, 41, 982-986.

Martina, B. E. E., Koraka, P., \& Osterhaus, A. D. M. E. (2009). Dengue Virus Pathogenesis: an Integrated View. Clinical Microbiology Reviews, 22, 564-581.

McBride, W. J., \& Bielefeldt-Ohmann, H. (2000). Dengue viral infections; pathogenesis and epidemiology. Microbes and infection, 2, 1041-1050.

Miller, B.R., DeFoliart, G.R., \& Yuill. T.M. (1978). Infection rates of Aedes triseriatus following ingestion of La Crosse virus by the larvae. American Journal Tropical Medicine and Hygiene, 27, 605-608.

Medeiros, L. C. de C., Castilho, C. A. R., Braga, C., de Souza, W. V., Regis, L., \& Monteiro, A. M. V. (2011). Modeling the Dynamic Transmission of Dengue Fever: Investigating Disease Persistence. PLoS Neglected Tropical Diseases, 5, e942.

Moll, R.M., Romoser, W.S., Moncayo, A.C., \& Lerdthusnee, K. (2001). Meconial peritrophic membranes and the fate of midgut bacteria during mosquito (Diptera: Culicidae) metamorphosis. Journal of Medical Entomology. 3, 29-32.

Moncayo, A.C., Fernandez, Z., Ortiz, D., Diallo, M., Sall, A., Hartman, S., Davis, T., Coffey, L., Mathiot, C.C., Tesh, R.B., and Weaver, S.C. (2004). Dengue emergence and adaptation to peridomestic mosquitoes. Emerging Infectious Diseases, 10, 1790-1796

Mourya, D. T., Gokhale, Basu, A., Barde, P. V., Sapkal, G. N., Padbidri, V. S., \& Gore, M. M. (2001). Horizontal and vertical transmission of dengue virus type 2 in highly and lowly susceptible strains of Aedes aegypti mosquitoes. Acta Virologica, 
$45,67-71$

Murrell, E. G., \& Juliano, S. A. (2008). Detritus type alters the outcome of interspecific competition between Aedes aegypti and Aedes albopictus (Diptera: Culicidae). Journal of Medical Entomology, 45, 375.

Murrell, E. G., Damal, K., Lounibos, L. P., \& Juliano, S. A. (2011). Distributions of Competing Container Mosquitoes Depend on Detritus Types, Nutrient Ratios, and Food Availability. Annals of the Entomological Society of America, 104, 688-698.

Muturi, E. J., \& Alto, B. W. (2011). Larval Environmental Temperature and Insecticide Exposure Alter Aedes aegypti Competence for Arboviruses. Vector-Borne and Zoonotic Diseases, 11, 1157-1163.

Muturi, E. J., Blackshear, M., \& Montgomery, A. (2012). Temperature and densitydependent effects of larval environment on Aedes aegypti competence for an alphavirus. Journal of Vector Ecology, 37, 154-161.

Muturi, E. J., Costanzo, K., Kesavaraju, B., \& Alto, B. W. (2011a). Can Pesticides and Larval Competition Alter Susceptibility of Aedes mosquitoes (Diptera: Culicidae) to Arbovirus Infection? Journal of Medical Entomology, 48, 429-436.

Muturi, E. J., Kim, C. H., Alto, B. W., Berenbaum, M. R., \& Schuler, M. A. (2011b). Larval environmental stress alters Aedes aegypti competence for Sindbis virus. Tropical Medicine \& International Health, 16, 955-964.

Nasci, R. S. (1991). Influence of larval and adult nutrition on biting persistence in Aedes aegypti (Diptera: Culicidae), Journal of Medical Entomology, 28, 522-526.

O'Donnell, D., \& Armbruster, P. (2007). Comparison of Larval Foraging Behavior of Aedes albopictus and Aedes japonicus (Diptera: Culicidae). Journal of Medical 
Entomology, 44, 984-989.

OhAinle, M., Balmaseda, A., Macalalad, A. R., Tellez, Y., Zody, M. C., Saborio, S., Nunez, A., et al. (2011). Dynamics of Dengue Disease Severity Determined by the Interplay Between Viral Genetics and Serotype-Specific Immunity. Science Translational Medicine, 3

Ooi, E., \& Gubler, D.J. (2010). Dengue-virus mosquito interactions. Frontiers in dengue virus research.

Patsoula, E., Samanidou-Voyadjoglou, A., Spanakos, G., Kremastinou, J., Nasioulas, G., \& Vakalis, N. C. (2006). Molecular and Morphological Characterization of Aedes albopictusin Northwestern Greece and Differentiation from Aedes cretinus and Aedes aegypti. Journal of Medical Entomology, 43, 40-54.

Paupy, C., Delatte, H., Bagny, L., Corbel, V., \& Fontenille, D. (2009). Aedes albopictus, an arbovirus vector: From the darkness to the light. Microbes and Infection, 11, $1177-1185$.

Peleg, J. (1965). Infection of mosquito larvae by arboviruses. American Journal Tropical Medicine Hygiene, 14, 158-164.

Pelz-Stelinski, K. S., Walker, E. D., \& Kaufman, M. G. (2010). Senescent leaf exudate increases mosquito survival and microbial activity. Ecological Entomology, 35, $329-340$.

Ponlawat, A., \& Harrington, L. C. (2007). Age and Body Size Influence Male Sperm Capacity of the Dengue Vector Aedes aegypti (Diptera: Culicidae). Journal of Medical Entomology, 44, 422-426.

Reiskind, M. H., \& Lounibos, L. P. (2009). Effects of intraspecific larval competition on 
adult longevity in the mosquitoes Aedes aegypti and Aedes albopictus. Medical and Veterinary Entomology, 23, 62-68.

Reiskind, M. H., \& Zarrabi, A. A. (2012). Is bigger really bigger? Differential responses to temperature in measures of body size of the mosquito, Aedes albopictus. Journal of Insect Physiology, 58, 911-917.

Reiskind, M. H., Westbrook, C. J., \& Lounibos, L. P. (2010). Exposure to chikungunya virus and adult longevity in Aedes aegypti (L.) and Aedes albopictus (Skuse). Journal of Vector Ecology, 35, 61-68.

Rosen, L., D.A. Shroyer, R.B. Tesh, J.E. Freier, and J.C. Lien. (1983). Transovarial transmission of dengue viruses by mosquitoes: Aedes albopictus and Aedes aegypti. American Journal Tropical Medicine Hygiene. 32, 1108-1119.

Rosen, L. (1987a). Sexual transmission of dengue viruses by Aedes albopictus. American Journal of Tropical Medicine and Hygiene. 37, 398-402.

Rosen, L. (1987b). Mechanism of vertical transmission of the dengue virus in mosquitoes. Comptes rendus de l'Académie des sciences. Série III, Sciences de la vie, 304, $347-350$.

Rosen, L. (1988). Further observations on the mechanism of vertical transmission of flaviviruses by Aedes mosquitoes. American Journal of Tropical Medicine and Hygiene, 39, 123-126.

Salazar, M. I., Richardson, J. H., Sanchez-Vargas, I., Olson, K. E., \& Beaty, B. J. (2007). Dengue virus type 2: replication and tropisms in orally infected Aedes aegypti mosquitoes. BMC Microbiology, 7, 9.

San Martin, J. L., Brathwaite, O., Zambrano, B., Solorzano, J. O., Bouckenooghe, A., 
Dayan, G. H., \& Guzman, M. G. (2010). The Epidemiology of Dengue in the Americas Over the Last Three Decades: A Worrisome Reality. American Journal of Tropical Medicine and Hygiene, 82, 128-135.

Schmid-Hempel, P. (2005). Evolutionary Ecology of Insect Immune Defenses. Annual Review of Entomology, 50, 529-551.

Schneider, J., Mori, A., Romero Severson, J., Chadee, D., \& Severson, D. (2007). Investigations of dengue-2 susceptibility and body size among Aedes aegypti populations. Medical and Veterinary Entomology, 21, 370-376.

Scott, T. W., \& Lorenz, L. H. (1998). Reduction of Culiseta melanura fitness by eastern equine encephalomyelitis virus. American Journal of Tropical Medicine and Hygiene, 59, 341-346.

Scott, T. W., Amerasinghe, P. H., Morrison, A. C., Lorenz, L. H., Clark, G. G., Strickman, D., Kittayapong, P., et al. (2000). Longitudinal Studies of Aedes aegypti (Diptera: Culicidae) in Thailand and Puerto Rico: Blood Feeding Frequency. Journal of Medical Entomology, 37, 89-101.

Shroyer, D. A. (1990). Vertical maintenance of dengue-1 virus in sequential generations of Aedes albopictus. Journal of the American Mosquito Control Association, 6, $312-314$.

Shurtleff, A. C., Beasley, D. W. C., Chen, J. J. Y., Ni, H., Suderman, M. T., Wang, H., Xu, R., et al. (2001). Genetic Variation in the 3' Non-Coding Region of Dengue Viruses. Virology, 281, 75-87.

Sithisarn, P., Suksanpaisan, L., Thepparit, C., \& Smith, D. R. (2003). Behavior of the dengue virus in solution. Journal of Medical Virology, 71, 532-539. 
Styer, L. M., Carey, J. R., Wang, J.L., \& Scott, T. W. (2007a). Mosquitoes do senesce: departure from the paradigm of constant mortality. American Journal of Tropical Medicine and Hygiene, 76, 111-117.

Styer, L. M., Meola, M. A., \& Kramer, L. D. (2007b). West Nile virus infection decreases fecundity of Culex tarsalis females. Journal of Medical Entomology, 44, 1074-1085.

Sumanochitrapon, W., Strickman, D., Sithiprasasna, R., Kittayapong, P., \& Innis, B. L. (1998). Effect of size and geographic origin of Aedes aegypti on oral infection with dengue-2 virus. American Journal of Tropical Medicine and Hygiene, 58, 283-286.

Suwanchaichinda, C., \& Paskewitz, S. M. (1998). Effects of larval nutrition, adult body size, and adult temperature on the ability of Anopheles gambiae (Diptera: Culicidae) to melanize Sephadex beads. Journal of Medical Entomology, 35, $157-$ 161.

Takken, W., Constantini, C., Dolo, G., Hassanali, A., Sagnon, N., Osir, E. (2006). Mosquito mating behavior. In Bridging Laboratory and Field Research for Genetic Control of Disease Vectors Edited by: Knols BGJ, Louis C. Netherlands: Springer;:183-188.

Telang, A., Qayum, A. A., Parker, A., Sacchetta, B. R., \& Byrnes, G. R. (2011). Larval nutritional stress affects vector immune traits in adult yellow fever mosquito Aedes aegypti (Stegomyia aegypti). Medical and Veterinary Entomology, 26, 271-281.

Thenmozhi, V., Hiriyan, J. G., Tewari, S. C., Philip Samuel, P., Paramasivan, R., Rajendran, R., Mani, T. R., et al. (2007). Natural vertical transmission of dengue 
virus in Aedes albopictus (Diptera: Culicidae) in Kerala, a southern Indian state. Japanese Journal of Infectious Diseases, 60, 245-249.

Tsetsarkin, K.A., D.L. Vanlandingham, C.E. McGee, and S. Higgs. (2007). A Single Mutation in Chikungunya Virus Affects Vector Specificity and Epidemic Potential. PLoS Pathogens 3, e201.

Tun-Lin, W., Burkot, T. R., \& Kay, B. H. (2000). Effects of temperature and larval diet on development rates and survival of the dengue vector Aedes aegypti in north Queensland, Australia. Medical and Veterinary Entomology, 14, 31-37.

Turell, M.J., K.J. Linthicum, \& J.R. Beaman. (1990). Transmission of Rift Valley Fever virus by adult mosquitoes after ingestion of virus as larvae. American Journal of Tropical Medicine and Hygiene. 43, 677-680.

Vasilakis, N., Shell, E.J., Fokam, E.B., Mason, P.W., Hanley, K.A., Estes, D.M., \& Weaver, S.C. (2006). Potential of ancestral sylvatic dengue-2 viruses to re-emerge. Virology, 358, 402-412.

Vasilakis, N., \& Weaver, S. C. (2008). Chapter 1 - The History and Evolution of Human Dengue Emergence. Advances in Virus Research (pp. 1-76). Elsevier Inc.

Vasilakis, N., Durbin, A. P., da Rosa, A. P. A. T., Munoz-Jordan, J. L., Tesh, R. B., \& Weaver, S. C. (2008). Antigenic relationships between sylvatic and endemic dengue viruses. American Journal of Tropical Medicine and Hygiene, 79, 128-132.

Vasilakis, N., Cardosa, J., Hanley, K. A., Holmes, E. C., \& Weaver, S. C. (2011). Fever from the forest: prospects for the continued emergence of sylvatic dengue virus and its impact on public health. Nature Reviews Microbiology, 9, 532-541.

Watts, D.M., \& Eldridge, B.F. (1975). Transovarial transmission of arboviruses by 
mosquitoes: a review. Medical Biology, 53, 271-278.

Wang, E., Ni, H., Xu, R., Barrett, A. D. T., Watowich, S. J., Gubler, D. J., \& Weaver, S. C. (2000). Evolutionary relationships of endemic/epidemic and sylvatic dengue viruses. Journal of Virology, 74, 3227-3234.

Weaver, S.C., \& Vasilakis, N. (2009). Molecular evolution of dengue viruses: contributions of phylogenetics to understanding the history and epidemiology of the preeminent arboviral disease. Infection, Genetics and Evolution, 9, 523-540

Weaver, S. C., \& Reisen, W. K. (2010). Present and future arboviral threats. Antiviral Research, 85, 328-345.

Westbrook, C. J., Reiskind, M. H., Pesko, K. N., Greene, K. E., \& Lounibos, L. P. (2012). Larval environmental temperature and the susceptibility of Aedes albopictus Skuse (Diptera: Culicidae) to Chikungunya virus. Vector-Borne and Zoonotic Diseases, $10,241-247$.

Whitehead, S. S., Blaney, J. E., Durbin, A. P., \& Murphy, B. R. (2007). Prospects for a dengue virus vaccine. Nature Reviews Microbiology, 5, 518-528.

Wong, J., Morrison, A. C., Stoddard, S. T., Astete, H., Chu, Y. Y., Baseer, I., \& Scott, T. W. (2012). Linking Oviposition Site Choice to Offspring Fitness in Aedes aegypti: Consequences for Targeted Larval Control of Dengue Vectors. PLoS Neglected Tropical Diseases, 6, e1632.

Yee, D. A. (2008). Tires as Habitats for Mosquitoes: A Review of Studies Within the Eastern United States. Journal of Medical Entomology, 45, 581-593.

Yee, D. A., \& Juliano, S. A. (2006). Consequences of detritus type in an aquatic microsystem: effects on water quality, microorganisms and performance of the 
dominant consumer. Freshwater Biology, 51, 448-459.

Yee, D. A., \& Juliano, S. A. (2011). Concurrent effects of resource pulse amount, type, and frequency on community and population properties of consumers in detritus based systems. Oecologia, 169, 511-522.

Yee, D. A., Allgood, D., Kneitel, J. M., \& Kuehn, K. A. (2012). Constitutive Differences between Natural and Artificial Container Mosquito Habitats: Vector Communities, Resources, Microorganisms, and Habitat Parameters. Journal of Medical Entomology, 49, 482-491.

Yee, D. A., Kaufman, M. G., \& Juliano, S. A. (2007a). The significance of ratios of detritus types and micro-organism productivity to competitive interactions between aquatic insect detritivores. Journal of Animal Ecology, 76, 1105-1115.

Yee, D. A., Kesavaraju, B., \& Juliano, S. A. (2007b). Direct and indirect effects of animal detritus on growth, survival, and mass of invasive container mosquito Aedes albopictus (Diptera: Culicidae). Journal of Medical Entomology, 44, 580588.

Zeidler, J. D., Acosta, P. O. A., Barrêto, P. P., \& Cordeiro, J. D. S. (2008). Dengue virus in Aedes aegypti larvae and infestation dynamics in Roraima, Brazil. Revista de saúde pública, 42, 986-991.

Zhang, M., Zheng, X., Wu, Y., Gan, M., He, A., Li, Z., Liu, J., et al. (2010). Quantitative analysis of replication and tropisms of dengue virus type 2 in aedes albopictus. American Journal of Tropical Medicine and Hygiene, 83, 700-707.

Zuk, M. \& Stoehr, A.M. (2002). Immune defense and host life history. The American Naturalist, 160, S9-S22. 


\title{
CURRICULUM VITAE
}

\author{
Name: $\quad$ Jeffrey J. Bara \\ Address: $\quad 5801 \mathrm{~S}$. Watterson Trail \\ Louisville, KY 40291 \\ Phone: (502) 345-6607 \\ E-mail: jay.bara@gmail.com \\ DOB: $\quad$ Oak Park, IL - December 3, 1984
}

\section{Education:}

Gradutae: 2007-2012

Degree: $\quad \mathrm{PhD}$ in Molecular, Cellular, and Developmental Biology

School: $\quad$ University of Louisville

GPA: $\quad 3.60$

Undergraduate: 2003-2007

Degree: $\quad$ B.S., Biology

School: Western Illinois University

GPA: $\quad 3.28$

\section{Teaching:}

Laboratory Instructor, Department of Biology, University of Louisville, Louisville, KY August 2009 - present

Taught laboratory sections for undergraduate introductory microbiology course.

\section{Research}

Research Assistant, Department of Biology, Western Illinois University, Macomb, IL June 2006 - May 2007

Investigated the impact of burned and sucessional habitats on wasp and ground beetle diversity. Research responsibilities included setting and collecting pitfall and malaise traps, collecting and sorting specimens, and analyzing data.

Research Assistant, Department of Psychology, Western Illinois University, Macomb, IL June 2006 - May 2007 
Research was focused on understanding the fear-learning pathway and memory acquistion. I assisted on a pre-pulse inhibition trail to measure memory acquistion. Main laboratory duties include intra-amygdaloidal lesion surgeries using a sterotaxic appartatus, handling rats, and conducting experimental trials.

Pre-Doctoral Fellow/Ph.D. Research, Department of Biology, University of Louisville Louisville, KY August 2007 - present My doctoral research investigates interactions between dengue virus and larval mosquitoes, along with aspects of vector ecology. The questions I have investigating for my dissertation are:

- Are larval Ae. aegypti and Ae. albopictus susceptible to dengue virus?

- Can dengue virus be transmitted among Ae. aegypti and Ae. albopictus larvae?

- What is the fitness cost of dengue replication in larval Ae. aegypti and Ae. albopictus How does animal detritus influence population growth and competition in Ae. aegypti and Ae. albopictus?

- How do different detritus resources in the larval habiat influence larval development and competition among Ae. aegypti and Ae. albopictus?

- Vertical transmission of dengue virus in Aedes mosquitoes. (review)

\section{Publications}

Peer-reviewed

1. McCravy KW, Bara JJ, Hessler SN, Luxmore LK, Stinebaker KS, Jenkins SE. (2009) Abundance and Diversity of Thread-Waisted Wasps (Hymenoptera: Sphecidae: Sphecinae) at Alice L. Kibbe Life Science Station, Hancock County, Illinois USA. Transactions of the Illinois State Academy of Science 102: 107-115.

2. Bara, J.J., T.M. Clark, and S.K. Remold. Susceptibility of larval and Aedes albopictus to dengue virus. (Accepted 10/12) to Journal of Medical Entomology

In Preparation

Bara, J.J., T.M. Clark, and S.K. Remold. Consequences of Dengue replication within larval Aedes aegypti and Aedes albopictus mosquitoes. Research article for submission to PLoS NTD.

Bara, J.J. Vertical transmission of dengue virus by Aedes mosquitoes. Review article for submission to Tropical Medicine and International Health.

\section{Poster Presentations}

Year

2007 Blankenship, M., Crull, M., Bara J., Davis, J., and McFadden, S.L. The role of glucocorticoids in the amygdala during prepulse inhibition. Annual Meeting of Chicago Chapter of the Society for Neuroscience, DePaul University, March 23. 
Bara, J.J., S. K. Remold. Horizontal Transmission of Dengue Virus among Aedes aegypti and Aedes albopictus Larvae. Research! Louisville

Bara, J.J., S. K. Remold. Horizontal Transmission of Dengue Virus among Aedes aegypti and Aedes albopictus Larvae. Gordon Research Conference on Microbial Population Biology. Andover, NH

Bara, J.J., S.K. Remold. Susceptibility of Larval Aedes aegypti to Dengue Virus American Society for Microbiology. New Orleans, LA.

Eid.M.E., J. Bara, S.K Remold. The Effects of Larval Detritus on Aedes aegypti Development. University of Louisville Undergraduate Research Symposium.

Bara, J.J., M.E. Eid, S.K. Remold. Consequences of Dengue replication within larval Aedes aegypti and Aedes albopictus mosquitoes. Gordon Research Conference on Microbial Population Biology. Andover, NH

\section{Oral Presentations}

2011

Departmental seminar at Indiana University South Bend. Dengue virus mosquito interactions.

Plenary speaker at Western Illinois University's graduate research symposium Dengue virus mosquito interactions.

University of Louisville graduate research symposium. Susceptibility of Aedes

2012 aegypti to dengue virus

University of Louisville graduate research symposium. Vertical Transmission of dengue virus in Aedes mosquitoes

\section{Honors/ Professional Affiliations}

2007

2007-2009

2008-present

2009-present

2010-present

2010-present
William H. and Eva Little Graham scholarship. University Fellowship University of Louisville American Society of Microbiology Entomological Society of America Society of Vector Ecology American Society for Tropical Medicine 